\title{
Direct Catalytic Asymmetric Mannich-Type Reaction of Alkylamides
}

Fernando Arteaga Arteaga, Zijian Liu, Lennart Brewitz, Jianyang Chen, Bo Sun, Naoya Kumagai,* Masakatsu Shibasaki*

Institute of Microbial Chemistry (BIKAKEN), Tokyo, Japan

nkumagai@bikaken.or.jp, mshibasa@bikaken.or.jp

1. General methods

1-1. Reactions and purifications

1-2. Characterizations

$1-3$. Solvents and reagents

2. Synthesis and coordination properties of alkylamides

2-1. Synthesis and characterization of amides

2-2. NMR studies of mixtures of amide $1 \mathrm{a}$ and a Cu(I) complex

2-3. X-ray crystallographic analysis of acetamide 1e/Cu(I)/L1 complex

\section{General procedures}

3-1. Synthesis of imine $2 \boldsymbol{k}, 2 \boldsymbol{n}$

3-2. General procedure for anti-selective direct catalytic asymmetric Mannich-type reaction of propionamide 1 a and butyramide $1 d$

3-3. Characterization of anti-products

3-4. General procedure for syn-selective direct catalytic asymmetric Mannich-type reaction of propionamide $1 \mathrm{a}$ and butyramide $1 \mathrm{~d}$

Characterization of syn-products

3-6. General procedure for direct catalytic asymmetric Mannich-type reaction of acetamide 1e

3-7. Characterization of acetamide products

4. Determination of absolute configuration $\quad 22$

4-1. anti-Mannich adduct

4-3. Acetamide Mannich adduct

\begin{tabular}{lr} 
5. Transformation of product $3 g$ & 24 \\
\hline
\end{tabular}

6. References 26 


\section{General methods}

\section{1-1. Reactions and purifications}

Unless otherwise noted, all reactions were carried out in an oven-dried glassware fitted with a 3-way glass stopcock under an argon atmosphere with magnetically stirred chips. All work-up and purification procedures were carried out with reagent-grade solvents under ambient atmosphere. Thin layer chromatography (TLC) and preparative Thin layer chromatography (PTLC) were performed on Merck TLC plates $(0.25 \mathrm{~mm})$ and $(0.5 \mathrm{~mm})$ pre-coated with silica gel 60 F254 and visualized by UV quenching and staining with ninhydrin, $\mathrm{KMnO}_{4}$, anisaldehyde, and ceric ammonium molybdate solution. Flash column chromatography was performed with $\mathrm{SiO}_{2}$ Merck 60 (230-400 mesh), or Kanto Chemical 60N (neutral, spherical, 50-60 $\mu \mathrm{m}$ ), or Wakogel ${ }^{\circledR} 50 \mathrm{NH}_{2}$.

\section{1-2. Characterizations}

Infrared (IR) spectra were recorded on a HORIBA FT210 Fourier transform infrared spectrophotometer. NMR spectra were recorded on a JEOL ECS-400, a Bruker AVANCE III HD400 or a Bruker AVANCE III 500. Chemical shifts ( $\delta)$ are given in ppm relative to residual solvent peaks; for ${ }^{1} \mathrm{H} \mathrm{NMR},\left(\mathrm{CDCl}_{3}: \delta=7.28 \mathrm{ppm} ; \mathrm{DMSO}-d_{6}: \delta=2.49\right.$ ppm; $\mathrm{MeCN}-d_{3}$ : $\delta=1.94 \mathrm{ppm})$; for ${ }^{13} \mathrm{C} \mathrm{NMR},\left(\mathrm{CDCl}_{3}: \delta=77.00 \mathrm{ppm}\right.$; DMSO- $\left.d_{6}: \delta=39.52 \mathrm{ppm} ; \mathrm{MeCN}-d_{3}: \delta=118.26,1.32 \mathrm{ppm}\right) .{ }^{1} \mathrm{Data}$ for ${ }^{1} \mathrm{H}$ NMR are reported as follows: chemical shift (multiplicity, coupling constants where applicable, number of hydrogens). Abbreviations are as follows: s (singlet), $d$ (doublet), $t$ (triplet), dd (doublet of doublet), dt (doublet of triplet), ddd (doublet of doublet of doublet), q (quartet), m (multiplet), br (broad). For ${ }^{13} \mathrm{C} \mathrm{NMR}$ in $\mathrm{D}_{2} \mathrm{O}$, chemical shifts are reported in the scale relative to DDS- $d_{6}(\delta 0.00 \mathrm{ppm})$. For ${ }^{19} \mathrm{~F}$ NMR, chemical shifts were reported in the scale relative to $\mathrm{PhCF}_{3}\left(\delta-62.7680 \mathrm{ppm}\right.$ in $\left.\mathrm{CDCl}_{3}\right)$ as an external reference. For ${ }^{11} \mathrm{~B} \mathrm{NMR}$, chemical shifts were reported in the scale relative to $\mathrm{BF}_{3} \cdot \mathrm{Et}_{2} \mathrm{O}\left(\delta 0.00 \mathrm{ppm}\right.$ in $\left.\mathrm{CDCl}_{3}\right)$ as an external reference. Single-crystal X-ray data were collected on a Rigaku R-AXIS RAPID II imaging plate area detector with graphite-monochromated $\mathrm{Cu}-\mathrm{K} \alpha$ radiation. Optical rotation was measured using a $2 \mathrm{~mL}$ cell with a $1.0 \mathrm{dm}$ path length on a JASCO polarimeter P-1030. High-resolution mass spectra were measured on a Thermo Fisher Scientific LTQ Orbitrap XL. HPLC analysis was conducted on a JASCO HPLC system equipped with Daicel chiral-stationary-phase columns $(\phi 0.46 \mathrm{~cm} \times 25 \mathrm{~cm})$.

\section{1-3. Solvents and reagents}

THF, DME, and diethyl ether were purified by passing through a solvent purification system (Glass Contour). 2,3-Dihydro-7-azaindole (for the synthesis of 1a,d,e) was either purchased (Sigma-Aldrich or Combi-Blocks, Inc.) or prepared from 7-azaindole (purchased from Ark Pharm, Inc. or Combi-Blocks, Inc.) by following literature procedures. $^{2}$ 1,2,3,4-tetrahydro-1,8-naphthyridine (for the synthesis of $\mathbf{1 b}$ ) was purchased from Ark Pharm Inc. 6,7,8,9-Tetrahydro-5H-pyrido[2,3-b]azepine (for the synthesis of 1c) was synthesized following the literature procedure. ${ }^{3}$ The aromatic N-Boc imines were prepared by following literature procedures. ${ }^{4}$ All other starting materials were used as supplied by commercial vendors or prepared by the method described in the corresponding references. 


\section{2-1. Synthesis and characterization of amides}

\section{1-(2,3-Dihydro-1H-pyrrolo[2,3-b]pyridin-1-yl)propan-1-one (1a)}

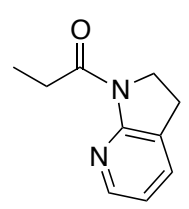

Propionyl chloride $(342.0 \mu \mathrm{L}, 3.92 \mathrm{mmol}, 1$ equiv) was added dropwise to a suspension of 2,3-dihydroazaindole (500 mg, $4.16 \mathrm{mmol}, 1.06$ equiv), $\mathrm{NaHCO}_{3}$ (610 mg, $8.3 \mathrm{mmol}, 2.1$ equiv) in $\mathrm{CH}_{2} \mathrm{Cl}_{2}$ $(15 \mathrm{~mL})$ at $0{ }^{\circ} \mathrm{C}$. The reaction mixture was warmed up to room temperature and stirred for $3 \mathrm{~h}$ before it was diluted with $\mathrm{CH}_{2} \mathrm{Cl}_{2}$ and water. The mixture was extracted with $\mathrm{CH}_{2} \mathrm{Cl}_{2}$ (3 times), dried over $\mathrm{Na}_{2} \mathrm{SO}_{4}$ and concentrated. The residue was purified by $\mathrm{SiO}_{2}$ chromatography (hexane/EtOAc, 2:1) to afford 430 mg $(62 \%)$ of amide 1a. X-ray crystallographic analysis revealed E-conformation in solid state, which is likely maintained in solution as indicated by relatively downfielded $\alpha$-proton via hydrogen bonding with the pyridyl nitrogen.

White solid, m.p.: $55-57{ }^{\circ} \mathrm{C} ;{ }^{1} \mathrm{H}$ NMR $\left(400 \mathrm{MHz}, 300 \mathrm{~K}, \mathrm{CDCl}_{3}\right): \delta 8.11(\mathrm{~d}, J=6.8 \mathrm{~Hz}, 1 \mathrm{H}), 7.47-7.44(\mathrm{~m}, 1 \mathrm{H}), 6.86(\mathrm{dd}, J=$ $7.2,4.8 \mathrm{~Hz}, 1 \mathrm{H}), 4.12(\mathrm{t}, J=8.8 \mathrm{~Hz}, 2 \mathrm{H}), 3.13(\mathrm{q}, J=7.2 \mathrm{~Hz}, 2 \mathrm{H}), 3.06(\mathrm{t}, J=8.8 \mathrm{~Hz}, 2 \mathrm{H}), 1.23(\mathrm{t}, J=7.2 \mathrm{~Hz}, 3 \mathrm{H}) ;{ }^{3} \mathrm{C} \mathrm{NMR}$ $\left(100 \mathrm{MHz}, 300 \mathrm{~K}, \mathrm{CDCl}_{3}\right): \delta 173.9,156.1,146.0,133.2,126.0,117.7,45.6,30.1,24.2,9.0 ; \mathrm{IR}(\mathrm{KBr}): \widetilde{v}=2976,2942,2925$, $1658,1600,1586,1483,1415,1236,1031 \mathrm{~cm}^{-1}$; HRMS (ESI) m/z calculated for $\mathrm{C}_{10} \mathrm{H}_{12} \mathrm{ON} \mathrm{N}_{2} \mathrm{Na}[\mathrm{M}+\mathrm{Na}]^{+}: 199.0842$, found: 199.0843. Refined structure and crystallographic parameters are summarized in Figure S1 and Table S1. CCDC 1469656 contains the supplementary crystallographic data for $\mathbf{1 a}$.

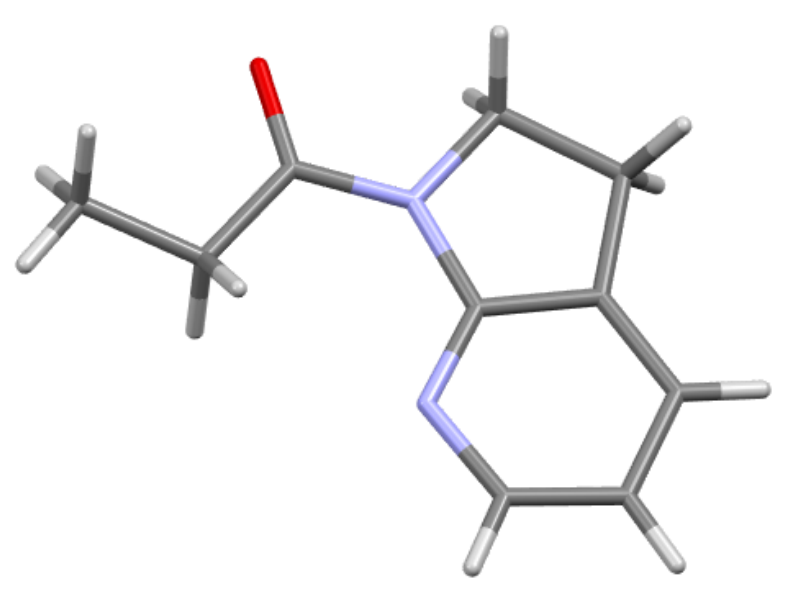

Figure S1. Structure of 1a. Color code; white: hydrogen, gray: carbon, blue: nitrogen, red: oxygen.
Table S1. Selected crystal data of 1a.

$\begin{array}{ll}\text { Empirical Formula } & \mathrm{C}_{20} \mathrm{H}_{24} \mathrm{O}_{2} \mathrm{~N}_{4} \\ \text { Formula Weight } & 352.24 \\ \text { Crystal Color, Habit } & \text { colorless, platelet } \\ \text { Crystal Dimensions } & 0.200 \times 0.200 \times 0.100 \mathrm{~mm} \\ \text { Crystal System } & \text { orthorhombic } \\ \text { Lattice Parameters } & \\ \text { a } & 13.6534(3) \AA \\ \text { b } & 6.92272(14) \AA \\ \text { c } & 18.5440(4) \AA \\ \text { V } & 1752.75(7) \AA \\ \text { Space Group } & P b c a \\ \text { Z value } & 4 \\ \text { Dcalc } & 1.335 \mathrm{~g} / \mathrm{cm}^{3} \\ \text { Foo } & 752.00\end{array}$

\section{1-(3,4-Dihydro-1,8-naphthyridin-1(2H)-yl)propan-1-one (1b)}<smiles>CCC(=O)N1CCCc2cccnc21</smiles>

Propionyl chloride (153.0 $\mu \mathrm{L}, 1.75 \mathrm{mmol}, 1.0$ equiv) was added dropwise to a suspension of 1,2,3,4-tetrahydro-1,8-naphthyridine (250 mg, $1.86 \mathrm{mmol}, 1.06$ equiv), $\mathrm{NaHCO}_{3}$ (309 mg, $3.68 \mathrm{mmol}, 2.1$ equiv), and $\mathrm{CH}_{2} \mathrm{Cl}_{2}(10 \mathrm{~mL})$ at $0{ }^{\circ} \mathrm{C}$. The reaction mixture was warmed up to ambient temperature and stirred for $3 \mathrm{~h}$ before it was diluted with water and extracted three times with $\mathrm{CH}_{2} \mathrm{Cl}_{2}$. The combined organic layers were dried over $\mathrm{Na}_{2} \mathrm{SO}_{4}$, filtered and evaporated. The residue was purified by flash column chromatography (hexane/ EtOAc, 2:1) to yield $205 \mathrm{mg}(62 \%)$ of $\mathbf{1 b}$.

Colorless oil. ${ }^{1} \mathrm{H}$ NMR $\left(400 \mathrm{MHz}, 300 \mathrm{~K}, \mathrm{CDCl}_{3}\right): \delta 8.19(\mathrm{dd}, J=4.8,1.6 \mathrm{~Hz}, 1 \mathrm{H}), 7.41(\mathrm{dt}, J=7.4,1.6 \mathrm{~Hz}, 1 \mathrm{H}), 6.97(\mathrm{dd}, J=$ $7.6,4.9 \mathrm{~Hz}, 1 \mathrm{H}), 3.87(\mathrm{dd}, J=6.2,6.2 \mathrm{~Hz}, 2 \mathrm{H}), 2.85(\mathrm{q}, J=7.4 \mathrm{~Hz}, 2 \mathrm{H}), 2.74(\mathrm{dd}, J=6.5,6.5 \mathrm{~Hz}, 2 \mathrm{H}), 1.91(\mathrm{~m}, 2 \mathrm{H}), 1.13(\mathrm{t}, J$ $=7.3 \mathrm{~Hz}, 3 \mathrm{H}) ;{ }^{13} \mathrm{C}$ NMR $\left(100 \mathrm{MHz}, 300 \mathrm{~K}, \mathrm{CDCl}_{3}\right): \delta$ 175.3, 152.0, 145.3, 136.9, 125.3, 119.6, 42.9, 30.1, 26.9, 23.0, 9.9; IR (film): $\tilde{v}=2937,1161,1574,1428,1371 \mathrm{~cm}^{-1}$; HRMS (ESI): $m / z$ calcd for $\mathrm{C}_{11} \mathrm{H}_{15} \mathrm{ON}_{2}[\mathrm{M}+\mathrm{H}]^{+}:$191.1179, found: 191.1180 . 


\section{1-(7,8-Dihydro-5H-pyrido[2,3-b]azepin-9(6H)-yl)propan-1-one (1c)}<smiles>CCC(=O)N1CCCCc2cccnc21</smiles>

6,7,8,9-Tetrahydro-5H-pyrido[2,3-b]azepine was converted to amide $1 \mathrm{c}$ by following the same procedure for 1d: propionyl chloride (37 $\mu \mathrm{L}, 423 \mu \mathrm{mol}, \quad 1.5$ equiv), 6,7,8,9-tetrahydro-5H-pyrido[2,3-b]azepine ( $41.8 \mathrm{mg}, 282 \mu \mathrm{mol}, 1.0$ equiv), Et $\mathrm{t}_{3} \mathrm{~N}(78.9 \mu \mathrm{L}, 564 \mu \mathrm{mol}, 2.0$ equiv), and $\mathrm{CH}_{2} \mathrm{Cl}_{2}(6 \mathrm{~mL})$ at $0{ }^{\circ} \mathrm{C}$. The residue was purified by flash column chromatography (hexane/EtOAc, 2:1) to yield $55.2 \mathrm{mg}(96 \%)$ of 1c. X-ray crystallographic analysis revealed $E$-conformation in solid state. In solution, $E$ - and Z-conformation is in equilibrium and two conformers were observed in NMR at room temperature.

Colorless solid, m.p.: 77.5-79.5 ${ }^{\circ} \mathrm{C}$; ${ }^{1} \mathrm{H}$ NMR $\left(400 \mathrm{MHz}, 343 \mathrm{~K}, \mathrm{CD}_{3} \mathrm{CN}\right): \delta 8.30$ (dd, J = 4.9, $\left.2.0 \mathrm{~Hz}, 1 \mathrm{H}\right), 7.69-7.66(\mathrm{~m}, 1 \mathrm{H})$, $7.22(\mathrm{dd}, J=7.6,4.9 \mathrm{~Hz}, 1 \mathrm{H}), 3.62(\mathrm{brs}, 2 \mathrm{H}), 2.78-2.73(\mathrm{~m}, 2 \mathrm{H}), 2.14(\mathrm{q}, J=7.4 \mathrm{~Hz}, 2 \mathrm{H}), 1.83-1.75(\mathrm{~m}, 2 \mathrm{H}), 1.74-1.66(\mathrm{~m}$, 2H), $1.00(\mathrm{t}, J=7.6 \mathrm{~Hz}, 3 \mathrm{H}) ;{ }^{13} \mathrm{C}$ NMR $\left(100 \mathrm{MHz}, 343 \mathrm{~K}, \mathrm{CD}_{3} \mathrm{CN}\right): \delta$ 173.6, 157.3, 147.8, 140.1, 136.1, 124.1, 46.2, 34.1, 29.9, 28.7, 26.8, 9.9; IR (KBr): $\widetilde{v}=2936,1652,1574,1436,1395 \mathrm{~cm}^{-1}$; HRMS (ESI): $m / z$ calcd for $\mathrm{C}_{12} \mathrm{H}_{16} \mathrm{ON}_{2} \mathrm{Na}[\mathrm{M}+\mathrm{Na}]^{+}$: 227.1155, found: 227.1156. Refined structure and crystallographic parameters are summarized in Figure S2 and Table S2. CCDC 1469736 contains the supplementary crystallographic data for 1c.

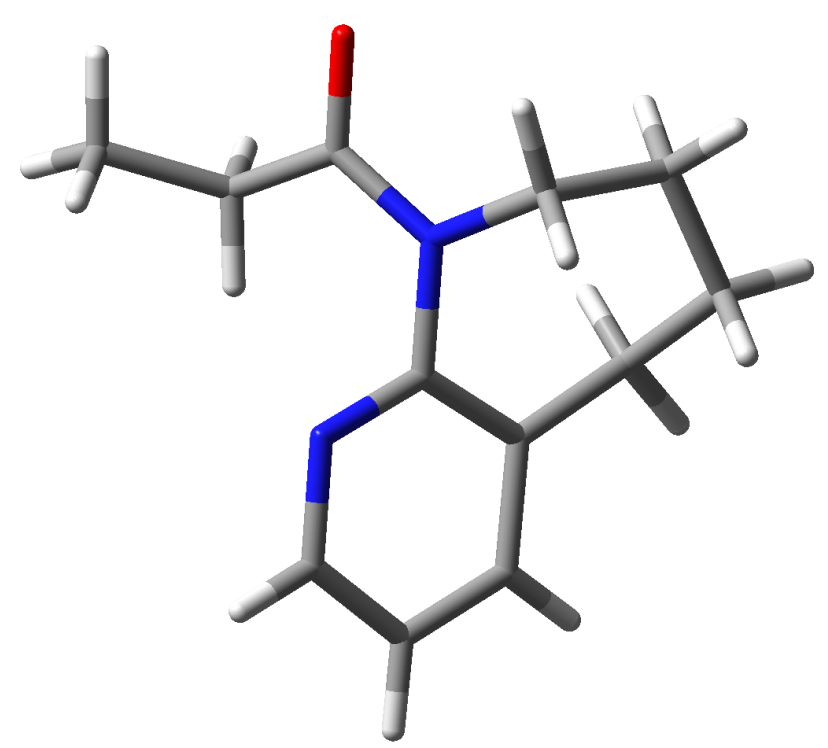

Table S2. Selected crystal data of $1 c$.

$\begin{array}{cl}\text { Empirical Formula } & \mathrm{C}_{12} \mathrm{H}_{16} \mathrm{ON}_{2} \\ \text { Formula Weight } & 204.27 \\ \text { Crystal Color, Habit } & \text { colorless, plate } \\ \text { Crystal Dimensions } & 0.200 \times 0.200 \times \\ \text { Crystal System } & \text { monoclinic } \\ \text { Lattice Parameters } & \\ \text { a } & 6.30143 \AA \\ \text { b } & 11.74261 \AA \\ \text { c } & 15.47710 \AA \\ \beta & 100.56100(7)^{\circ} \\ \text { V } & 1125.83180 \AA^{3} \\ \text { Space Group } & P 21 / \mathrm{c} \\ \text { Z value } & 4 \\ \text { Dcalc } & 1.205 \mathrm{~g} / \mathrm{cm}^{3} \\ \text { Fooo } & 440.00\end{array}$

Figure S2. Structure of 1c. Color code; white: hydrogen, gray: carbon, blue: nitrogen, red: oxygen.

\section{1-(2,3-Dihydro-1H-pyrrolo[2,3-b]pyridin-1-yl)butan-1-one (1d)}

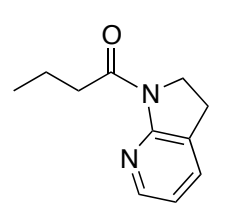

n-Butyryl chloride (313 $\mu \mathrm{L}, 3 \mathrm{mmol}, 1.5$ equiv) was added dropwise to a solution of 2,3-dihydro-7-azaindole ( $240.3 \mathrm{mg}, 2 \mathrm{mmol}, 1.0$ equiv), $\mathrm{Et} 3 \mathrm{~N}$ (558 $\mu \mathrm{L}, 4 \mathrm{mmol}, 2.0$ equiv), and $\mathrm{CH}_{2} \mathrm{Cl}_{2}(20$ $\mathrm{mL}$ ) at $0{ }^{\circ} \mathrm{C}$. The reaction mixture was warmed up to ambient temperature and stirred for $12 \mathrm{~h} . \mathrm{MeOH}$ $(2 \mathrm{~mL})$ was added and the reaction mixture was stirred for $30 \mathrm{~min}$ before removal of volatiles under reduced pressure. The residue was purified by flash column chromatography (hexane/EtOAc, 5:1) to yield $360 \mathrm{mg}(94 \%)$ of $\mathbf{1 d}$.

Colorless solid, m.p.: $59-61{ }^{\circ} \mathrm{C} ;{ }^{1} \mathrm{H}$ NMR $\left(400 \mathrm{MHz}, 300 \mathrm{~K}, \mathrm{CDCl}_{3}\right): \delta 8.10(\mathrm{~d}, J=4.9 \mathrm{~Hz}, 1 \mathrm{H}), 7.44(\mathrm{dd}, J=7.3,1.5 \mathrm{~Hz}, 1 \mathrm{H})$, $6.85(\mathrm{dd}, J=7.3,5.1 \mathrm{~Hz}, 1 \mathrm{H}), 4.10(\mathrm{dd}, J=8.6,8.6 \mathrm{~Hz}, 2 \mathrm{H}), 3.10(\mathrm{t}, J=7.5 \mathrm{~Hz}, 2 \mathrm{H}), 3.04(\mathrm{dd}, J=8.6,8.6 \mathrm{~Hz}, 2 \mathrm{H}), 1.74(\mathrm{~m}$, 2H), $1.01(\mathrm{t}, J=7.3 \mathrm{~Hz}, 3 \mathrm{H}) ;{ }^{13} \mathrm{C}$ NMR $\left(100 \mathrm{MHz}, 300 \mathrm{~K}, \mathrm{CDCl}_{3}\right): \delta$ 173.2, 156.1, 146.1, 133.2, 126.0, 117.7, 45.5, 38.4, 24.2, 18.3, 14.0; IR (KBr): $\widetilde{v}=2964,1649,1588,1421,793 \mathrm{~cm}^{-1}$; HRMS (ESI): $\mathrm{m} / z$ calcd for $\mathrm{C}_{11} \mathrm{H}_{14} \mathrm{ON}_{2} \mathrm{Na}[\mathrm{M}+\mathrm{Na}]^{+}: 213.0098$, found: 213.0098 .

The synthesis and characterization of $1 \mathbf{e}$ are reported in the literature. ${ }^{5}$

Refined structure and crystallographic parameters of a single crystal of 1e are summarized in Figure S3 and Table S3. CCDC 1469655 contains the supplementary crystallographic data for $1 \mathbf{e}$. 


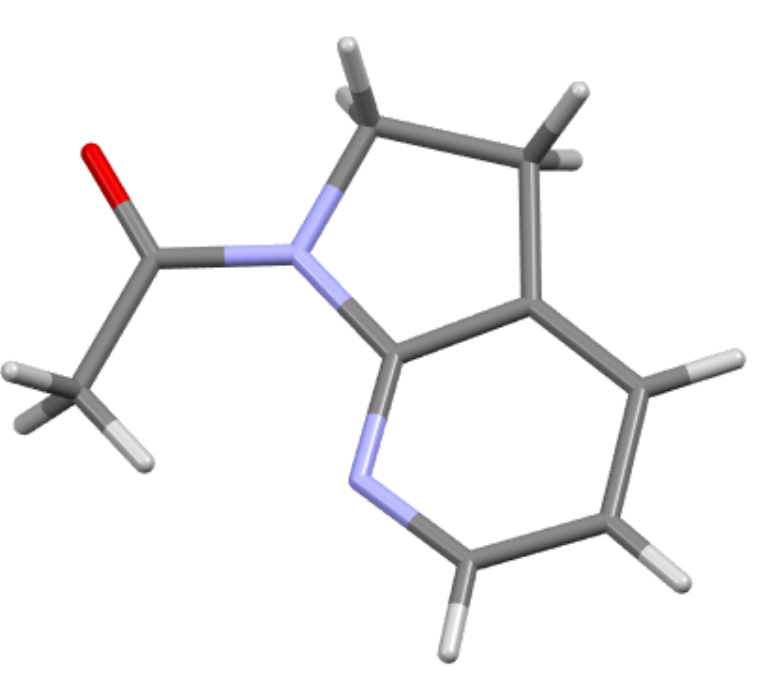

Figure S3. Structure of 1e. Color code; white: hydrogen, gray: carbon, blue: nitrogen, red: oxygen.

Table S3. Selected crystal data of 1e.

Empirical Formula

Formula Weight

Crystal Color, Habit

Crystal Dimensions

Crystal System

Lattice Parameters

$\begin{array}{ll}\mathrm{a} & 7.0381(2) \AA \\ \mathrm{b} & 13.3229(4) \AA \\ \mathrm{c} & 8.7353(3) \AA \\ \beta & 112.215(8){ }^{\circ} \\ \mathrm{V} & 758.30(6) \AA^{3} \\ \text { Space Group } & P 21 / \mathrm{c} \\ \text { Z value } & 4 \\ \text { D }_{\text {calc }} & 1.421 \mathrm{~g} / \mathrm{cm}^{3} \\ \text { Foo0 } & 344.00\end{array}$

$\mathrm{C}_{9} \mathrm{H}_{10} \mathrm{ON}_{2}$

162.19

colorless, platelet $0.200 \times 0.200 \times 0.100 \mathrm{~mm}$ monoclinic

\section{2-2. NMR studies of mixtures of amide $1 a$ and a $\mathrm{Cu}(\mathrm{I})$ complex}

A flame-dried $20 \mathrm{ml}$ test tube equipped with a magnetic stirring bar and three-way glass stopcock was charged with $\left[\mathrm{Cu}\left(\mathrm{CH}_{3} \mathrm{CN}\right)_{4}\right] \mathrm{PF}_{6}(19.6 \mathrm{mg}, 52.5 \mu \mathrm{mol}, 1.05$ equiv), (R)-xyl-BINAP (38.6 mg, $52.5 \mu \mathrm{mol}, 1.05 \mathrm{equiv})$, and THF- $d_{8}(0.6 \mathrm{~mL})$. The mixture was stirred for $1 \mathrm{~h}$ at room temperature and then transferred to another flamed-dried $20 \mathrm{~mL}$ tube containing $1 \mathrm{a}(8.8 \mathrm{mg}, 50 \mu \mathrm{mol}, 1.0$ equiv). After stirring $3 \mathrm{~min}$ at room temperature, the mixture was transferred to a

(a) Amide 1a in THF-d8

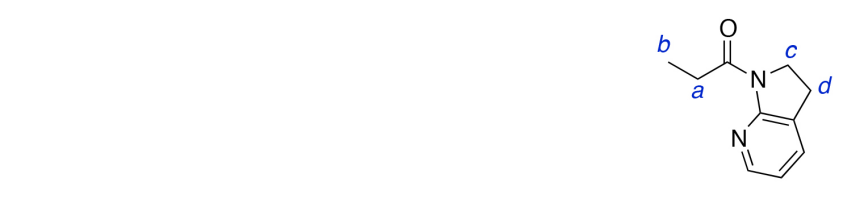

(b) Amide $1 \mathrm{a} /\left[\mathrm{Cu}\left(\mathrm{CH}_{3} \mathrm{CN}\right)_{4}\right] \mathrm{PF}_{6} / \mathrm{L} 1=1 / 1 / 1$ in THF-d8
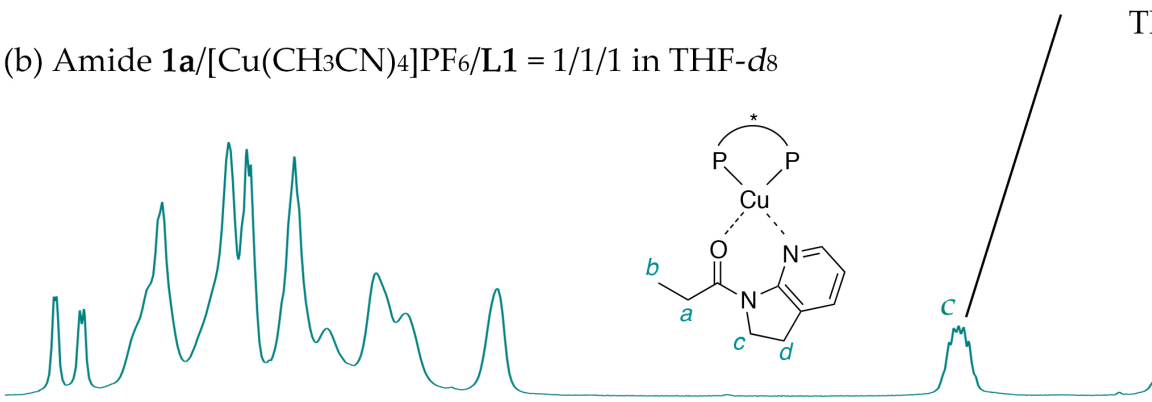

THF

(c) $\left[\mathrm{Cu}\left(\mathrm{CH}_{3} \mathrm{CN}\right)_{4}\right] \mathrm{PF} 6 / \mathrm{L} 1=1 / 1$ in THF-d8
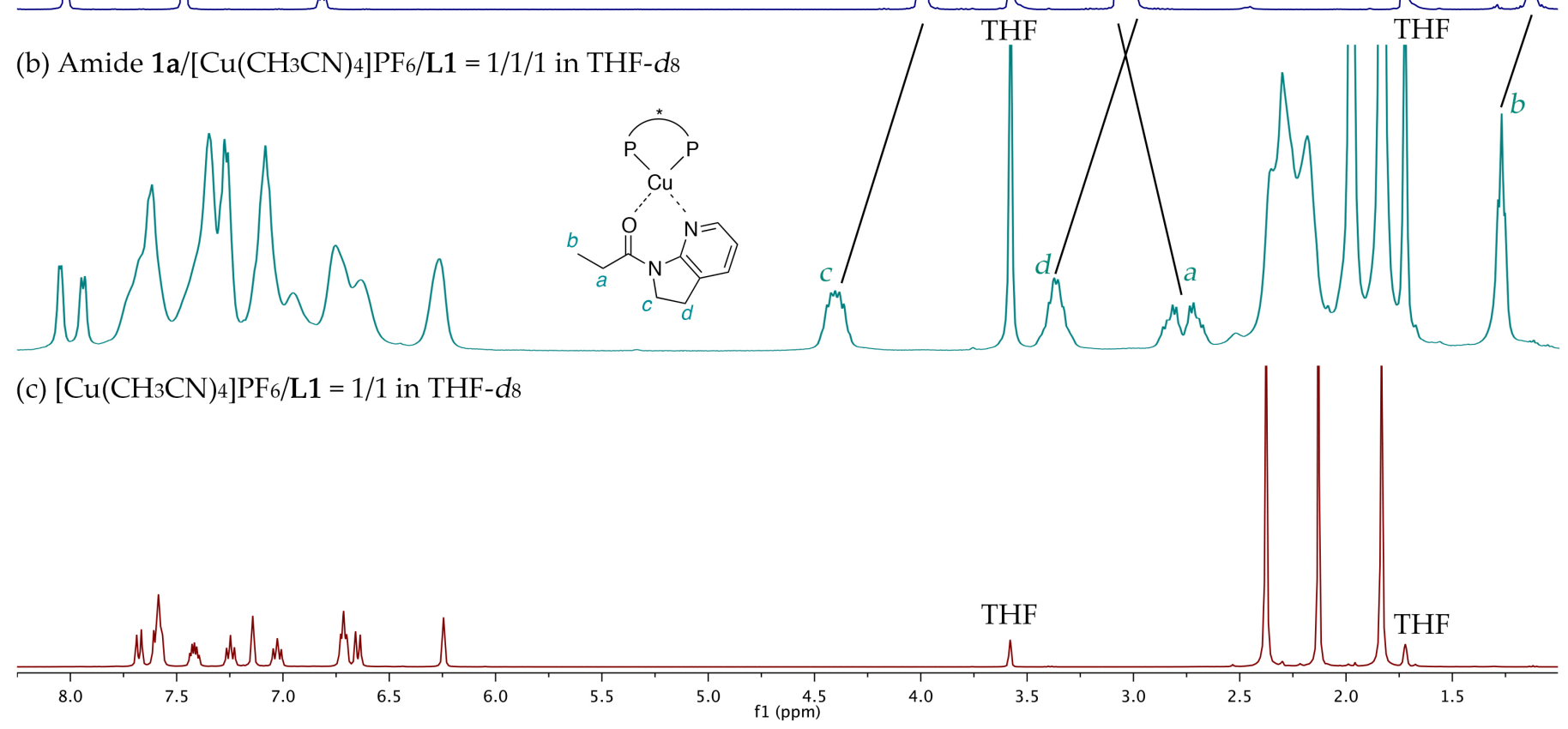
Figure S4. ${ }^{1} \mathrm{H}$ NMR Spectra of (a) amide $\mathbf{1 a}$ in THF- $d_{8}$, (b) amide $\mathbf{1 a} /\left[\mathrm{Cu}\left(\mathrm{CH}_{3} \mathrm{CN}\right)_{4}\right] \mathrm{PF}_{6} / \mathbf{L} \mathbf{1}$, and (c) $\left[\mathrm{Cu}\left(\mathrm{CH}_{3} \mathrm{CN}\right)_{4}\right] \mathrm{PF}_{6} / \mathbf{L} \mathbf{1}$ in THF-d8.

NMR tube under argon atmosphere, followed by NMR experiment. As shown in Figure S4, 1a/Cu(I)/L1 complex was formed in which the $\alpha$-proton was shifted upfield due to the lack of hydrogen bonding with the pyridyl nitrogen. This is ascribed to the bidentate coordination of $1 \mathrm{a}$ to $\mathrm{Cu}(\mathrm{I})$ via $\mathrm{Z}$-conformation.

\section{2-3. X-ray crystallographic analysis of acetamide $1 \mathrm{e} / \mathrm{Cu}(\mathrm{I}) / \mathrm{L1}$ complex}

A flame-dried $20 \mathrm{~mL}$ test tube equipped with a magnetic stirring bar and 3-way glass stopcock was charged with $\left[\mathrm{Cu}\left(\mathrm{CH}_{3} \mathrm{CN}\right)_{4}\right] \mathrm{PF}_{6}(18.6 \mathrm{mg}, 0.05 \mathrm{mmol}),(R)$-xyl-BINAP (L1) $(36.7 \mathrm{mg}, 0.05 \mathrm{mmol})$, and THF $(1.0 \mathrm{~mL})$. After the mixture was stirred for $1 \mathrm{~h}$ at room temperature, amide $1 \mathrm{e}(0.05 \mathrm{mmol}, 8.1 \mathrm{mg})$ was added to give a colorless solution, which was stirred for $30 \mathrm{~min}$ at room temperature. The mixture was transferred in glass vials to slowly grow crystals via vapor diffusion with $n$-hexane. Crystals were then directly collected from the vial and analyzed.

Single-crystal X-ray data were collected on a Rigaku R-AXIS RAPID II imaging plate area detector with graphite-monochromated $\mathrm{Cu}-\mathrm{Ka}$ radiation. Data collection was conducted at $93 \mathrm{~K}$. All structures were solved by direct methods and refined by full matrix least-squares against $F^{2}$ with all reflections. All non-hydrogen atoms were refined anisotropically. All hydrogen atoms were placed in standard calculated positions, and were refined using the riding model. Refined structure and crystallographic parameters are summarized in Figure S5 and Table S4. CCDC 1469659 contains the supplementary crystallographic data for the complex.

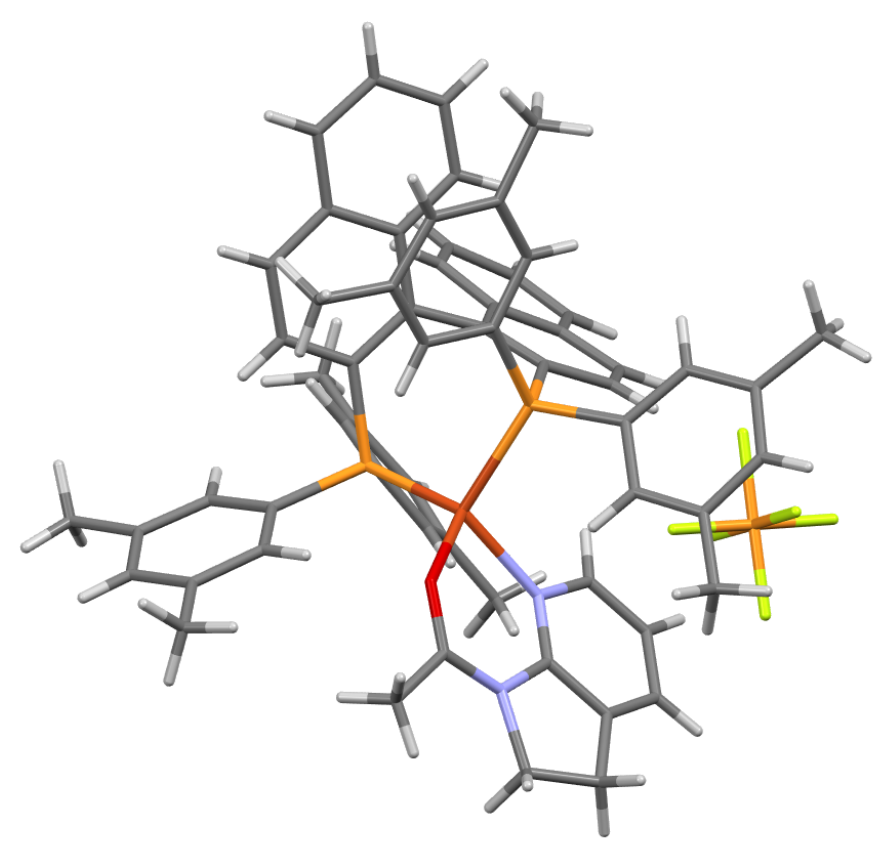

Figure S5. Structure of $\mathbf{1 e} / \mathrm{Cu}(\mathrm{I}) \mathrm{PF}_{6} / \mathbf{L 1}$. Color code; white: hydrogen, gray: carbon, blue: nitrogen, red: oxygen, green: fluorine, orange: phosphorus, dark orange: copper.

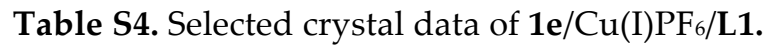
Empirical Formula Formula Weight $\mathrm{C}_{61} \mathrm{H}_{58} \mathrm{ON}_{2} \mathrm{~F}_{6} \mathrm{P}_{3}$ Crystal Color, Habit 1105.60 Crystal Dimensions colorless, platelet $0.200 \times 0.200 \times 0.100$ $\mathrm{mm}$

Crystal System monoclinic Lattice Parameters

$\begin{array}{ll}\mathrm{a} & 12.8125(2) \AA \\ \mathrm{b} & 15.5638(3) \AA \\ \mathrm{c} & 16.7344(3) \AA \\ \beta & 110.994(8){ }^{\circ} \\ \mathrm{V} & 3115.50(19) \AA^{3} \\ \text { Space Group } & P 21 \\ \text { Z value } & 2 \\ \text { D }_{\text {calc }} & 1.178 \mathrm{~g} / \mathrm{cm}^{3} \\ \text { Foo }_{00} & 692.00\end{array}$


Prepared by following the reported procedure. ${ }^{4 c}$ White solid. ${ }^{1} \mathrm{H}$ NMR $\left(400 \mathrm{MHz}, 300 \mathrm{~K}, \mathrm{CDCl}_{3}\right): \delta$ $=8.89(\mathrm{~s}, 1 \mathrm{H}), 8.35(\mathrm{~s}, 1 \mathrm{H}), 8.04-7.98(\mathrm{~m}, 2 \mathrm{H}), 7.49-7.46(\mathrm{~m}, 1 \mathrm{H}), 1.59(\mathrm{~s}, 9 \mathrm{H}), 1.35(\mathrm{brs}, 12 \mathrm{H}) ;{ }^{13} \mathrm{C}$ NMR (100 MHz, $\left.300 \mathrm{~K}, \mathrm{CDCl}_{3}\right): \delta$ 169.7, 162.6, 154.6, 139.7, 137.1, 133.4, 132.3, 128.2, 84.1, 82.1, 27.9, 24.9; ${ }^{11} \mathrm{~B}$ NMR $\left(128 \mathrm{MHz}, 300 \mathrm{~K}, \mathrm{CD}_{3} \mathrm{CN}\right): \delta=33.2$ (br); HRMS (ESI) $m / z$ calculated for $\mathrm{C}_{18} \mathrm{H}_{27} \mathrm{O}_{4} \mathrm{NB}$ $[\mathrm{M}+\mathrm{H}]^{+}:$332.2028, found: 332.2030. The imine obtained was in ca. $70 \%$ purity and further purification was intractable. 2 equiv. of imine was used for the reaction.

tert-Butyl ((1E,2E)-3-(4-fluorophenyl)allylidene)carbamate (2n)

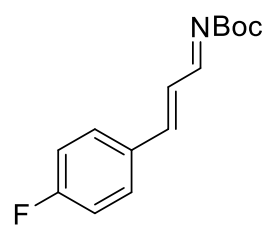

NBoc Prepared by following the reported procedure.4e White fluffy solid. ${ }^{1} \mathrm{H}$ NMR $(400 \mathrm{MHz}, 300 \mathrm{~K}$, $\left.\mathrm{CDCl}_{3}\right): \delta=8.69(\mathrm{~d}, J=9.2 \mathrm{~Hz}, 1 \mathrm{H}), 7.57-7.53(\mathrm{~m}, 2 \mathrm{H}), 7.33(\mathrm{~d}, J=16.0 \mathrm{~Hz}, 7.15-7.10(\mathrm{~m}, 2 \mathrm{H}), 6.91(\mathrm{dd}$, $J=16.0,9.2 \mathrm{~Hz}, 1 \mathrm{H}), 1.58(\mathrm{~s}, 9 \mathrm{H}) ;{ }^{13} \mathrm{C} \mathrm{NMR}\left(100 \mathrm{MHz}, 300 \mathrm{~K}, \mathrm{CDCl}_{3}\right): \delta 171.3,164.1(\mathrm{~d}, J=250.8 \mathrm{~Hz})$, 162.3, 149.0, 131.0, 130.1 (d, J = 8.7 Hz), 126.6, $116.3(\mathrm{~d}, J=21.9 \mathrm{~Hz}), 82.0,27.9$; ${ }^{9} \mathrm{~F}$ NMR $(376 \mathrm{MHz}$, $323 \mathrm{~K}, \mathrm{CD}_{3} \mathrm{CN}$ ): $\delta=-108.5$ (s, 1F); HRMS (ESI) $m / z$ calculated for $\mathrm{C}_{14} \mathrm{H}_{17} \mathrm{O}_{2} \mathrm{NFNa}[\mathrm{M}+\mathrm{Na}]^{+}:$272.1057, found: 272.1059 The imine obtained was in ca. $70 \%$ purity and further purification was intractable. 2 equiv. of imine was used for the reaction.

3-2. General procedure for anti-selective direct catalytic asymmetric Mannich-type reaction of propionamide 1a and butyramide $1 d$

A flame-dried $20 \mathrm{~mL}$ test tube equipped with a magnetic stirring bar and a 3-way glass stopcock were charged with (R)-trimethoxy-Biphep (L7) $(11.4 \mathrm{mg}, 0.012 \mathrm{mmol})$ and $\left[\mathrm{Cu}\left(\mathrm{CH}_{3} \mathrm{CN}\right)_{4}\right] \mathrm{PF}_{6}(3.8 \mathrm{mg}, 0.01 \mathrm{mmol})$ in a glove box. The test tube was removed from the glove box and evacuated for $10 \mathrm{~min}$. To the mixture was added THF $(0.1 \mathrm{~mL})$ via syringe with a stainless-steel needle at room temperature and under Ar atmosphere. The resulting clear solution was stirred for $1 \mathrm{~h}$. The corresponding amide $(0.1 \mathrm{mmol})$ and imine $(0.2 \mathrm{mmol})$ were then added. To the mixture cooled to $-10{ }^{\circ} \mathrm{C}$ was added Barton's base $(0.1 \mathrm{M}$ in THF, $0.1 \mathrm{~mL}, 0.01 \mathrm{mmol})$ via syringe with a stainless-steel needle. After $24 \mathrm{~h}$ of stirring, the reaction was quenched at $-10{ }^{\circ} \mathrm{C}$ with a solution of $\mathrm{NH} 4 \mathrm{Cl}$ aq. followed by extraction with EtOAc ( 3 times). Volatiles were removed under reduced pressure and the residue was purified by preparative TLC on silica gel with $\mathrm{CH}_{2} \mathrm{Cl}_{2} / \mathrm{Et}_{2} \mathrm{O} / \mathrm{EtOAc}(20: 1: 1)$ as an eluent to give desired product.

\section{3-3. Characterization of anti-products}

Note that the NMR spectra of the Mannich products were recorded at elevated temperature (usually $\left.50{ }^{\circ} \mathrm{C}\right)$ in $\mathrm{CD}_{3} \mathrm{CN}-d_{3}$, as rotamers were observed in $\mathrm{CDCl}_{3}$ at room temperature. ${ }^{6}$

Diastereomeric ratio (anti/syn) for compounds $3 a, 3 c-d, 3 f-h$ and $3 m$ were determined by ${ }^{1} H$ NMR analysis of the crude mixture $\left(\mathrm{CD}_{3} \mathrm{CN}-\mathrm{d}_{3}\right.$ at $\left.50^{\circ} \mathrm{C}\right)$, by integration of a characteristic downfield-shifted proton (anti $\delta \sim 8.10$ (d, J = 5.2 Hz, $1 H))$, (syn $\delta \sim 8.17(d, J=5.2 \mathrm{~Hz}, 1 \mathrm{H})$ ). For compounds $3 b, 3 e, 3 i-l, 3 n$ and $3 \mathrm{dg}$, the diastereomeric ratio was determined by HPLC analysis due to partially overlapping of diastereomers peaks in ${ }^{1} \mathrm{H}$ NMR spectrum.

\section{tert-Butyl ((1S,2R)-3-(2,3-dihydro-1H-pyrrolo[2,3-b]pyridin-1-yl)-2-methyl-3-oxo-1-phenylpropyl)carbamate} (anti-3a)

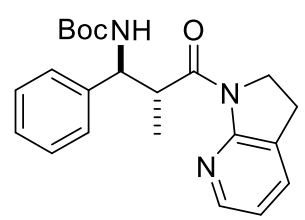

(94\% yield of mixture of diastereomers (anti/syn $=10 / 1), 36 \mathrm{mg})$. Anti-diastereomer was separated by PTLC. Amorphous solid; ${ }^{1} \mathrm{H}$ NMR $\left(400 \mathrm{MHz}, 323 \mathrm{~K}, \mathrm{CD}_{3} \mathrm{CN}\right): \delta=8.11(\mathrm{~d}, J=5.2 \mathrm{~Hz}$, $1 \mathrm{H}), 7.54-7.52(\mathrm{~m}, 1 \mathrm{H}), 7.33-7.16(\mathrm{~m}, 5 \mathrm{H}), 6.94(\mathrm{dd}, J=7.2,5.2 \mathrm{~Hz}, 1 \mathrm{H}), 6.64(\mathrm{br}, 1 \mathrm{H}), 4.91(\mathrm{br}, 1 \mathrm{H})$, $4.74(\mathrm{br}, 1 \mathrm{H}), 4.02-3.87(\mathrm{~m}, 2 \mathrm{H}), 3.03-2.86(\mathrm{~m}, 2 \mathrm{H}), 1.34(\mathrm{~s}, 9 \mathrm{H}), 1.12(\mathrm{~d}, J=6.8 \mathrm{~Hz}, 3 \mathrm{H}) ;{ }^{13} \mathrm{C}$ NMR

(100 MHz, $\left.323 \mathrm{~K}, \mathrm{CD}_{3} \mathrm{CN}\right): \delta 175.7,157.0,156.4,146.6,143.8,135.1,129.2,128.3,128.1,127.8,119.6$, 79.6, 59.6 (br), 47.2, 42.9, 28.7, 25.0, 16.2; IR (film): $\widetilde{v}=3411$ (br), 2975, 2929, 1713, 1646, 1590, 1494, 1421, 1304, 1167, 1084 $\mathrm{cm}^{-1}$; HRMS (ESI) $\mathrm{m} / z$ calculated for $\mathrm{C}_{22} \mathrm{H}_{28} \mathrm{O}_{3} \mathrm{~N}_{3} \mathrm{Na}[\mathrm{M}+\mathrm{Na}]^{+}: 404.1945$, found: 404.1940; $[\alpha]^{25} 39.0\left(c 0.3, \mathrm{CHCl}_{3}, 96 \%\right.$ ee sample); HPLC analysis CHIRALPAK IF $(\phi 0.46 \mathrm{~cm}$ x $25 \mathrm{~cm}), 2$-propanol $/ n$-hexane $=1 / 4$, flow rate $1.0 \mathrm{~mL} / \mathrm{min}$, detection at $254 \mathrm{~nm}, \mathrm{t}_{\mathrm{R}}=12.1 \mathrm{~min}$ (major), $19.7 \mathrm{~min}$ (minor). 

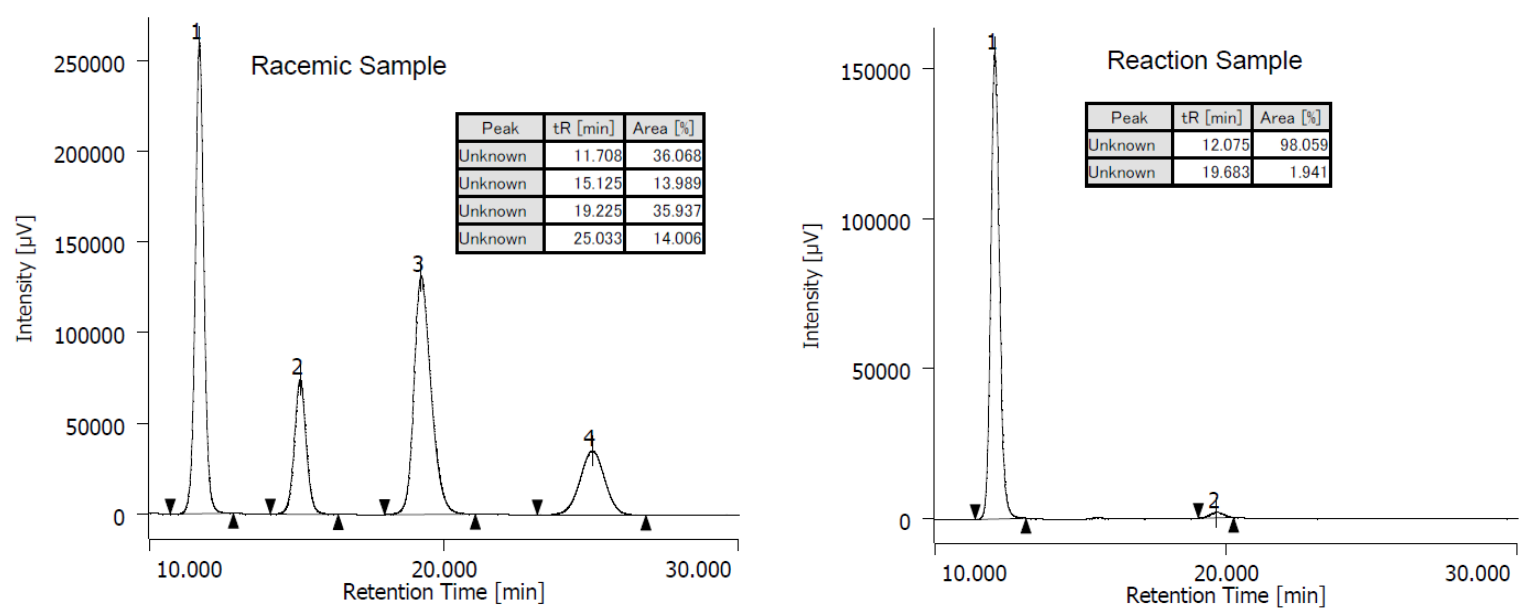

tert-Butyl ((1S,2R)-3-(2,3-dihydro-1H-pyrrolo[2,3-b]pyridin-1-yl)-2-methyl-3-oxo-1-(p-tolyl)propyl)carbamate (anti-3b)<smiles>Cc1ccc(C(NC(=O)OC(C)(C)C)[C@H](C)C(=O)N2CCc3cccnc32)cc1</smiles>

(89\% yield of mixture of diastereomers (anti/syn 16/1), $35 \mathrm{mg}$ ). Anti-diastereomer was separated by PTLC. White solid, m.p.: $129-131{ }^{\circ} \mathrm{C}$; ${ }^{1} \mathrm{H}$ NMR $(500 \mathrm{MHz}, 323 \mathrm{~K}, \mathrm{CD} 3 \mathrm{CN}): \delta=$ $8.10(\mathrm{~d}, J=5.0 \mathrm{~Hz}, 1 \mathrm{H}), 7.53(\mathrm{~d}, J=7.0 \mathrm{~Hz}, 1 \mathrm{H}), 7.19(\mathrm{~d}, J=7.5 \mathrm{~Hz}, 2 \mathrm{H}), 7.07(\mathrm{~d}, J=8.0 \mathrm{~Hz}, 2 \mathrm{H})$, $6.94(\mathrm{dd}, J=7.5,5.5 \mathrm{~Hz}, 1 \mathrm{H}), 6.61$ (brs, 1H), 4.87 (brs, 1H), 4.70 (brs, 1H), 4.00-3.88 (m, 2H), 3.01-2.86 (m, 2H), $2.25(\mathrm{~s}, 3 \mathrm{H}), 1.33(\mathrm{~s}, 9 \mathrm{H}), 1.11(\mathrm{~d}, J=6.5 \mathrm{~Hz}, 3 \mathrm{H}) ;{ }^{13} \mathrm{C}$ NMR $(125 \mathrm{MHz}, 323 \mathrm{~K}$, $\left.\mathrm{CD}_{3} \mathrm{CN}\right): \delta 175.7,157.0,156.4,146.6,140.7,137.6,135.0,129.8,128.3,127.7,119.5,79.5,59.2$ (br), 47.2, 42.9, 28.7, $24.9,21.1$, 16.2; IR (film): $\tilde{v}=3417$ (br), 2977, 1714, 1652, 1490, 1421, 1168, $1002 \mathrm{~cm}^{-1}$; HRMS (ESI) $\mathrm{m} / z$ calculated for $\mathrm{C}_{23} \mathrm{H}_{29} \mathrm{O}_{3} \mathrm{~N}_{3} \mathrm{Na}$ $[\mathrm{M}+\mathrm{Na}]^{+}:$418.2101, found: 418.2094; $[\alpha]_{\mathrm{D}}^{25} 45.3$ ( c 0.6, $\mathrm{CHCl}_{3}, 98 \%$ ee sample); HPLC analysis CHIRALPAK IF $(\phi 0.46 \mathrm{~cm}$ x $25 \mathrm{~cm}$ ), 2-propanol $/ n$-hexane $=1 / 4$, flow rate $1.0 \mathrm{~mL} / \mathrm{min}$, detection at $254 \mathrm{~nm}, \mathrm{t}=13.3 \mathrm{~min}$ (major), $21.1 \mathrm{~min}(\mathrm{minor})$.
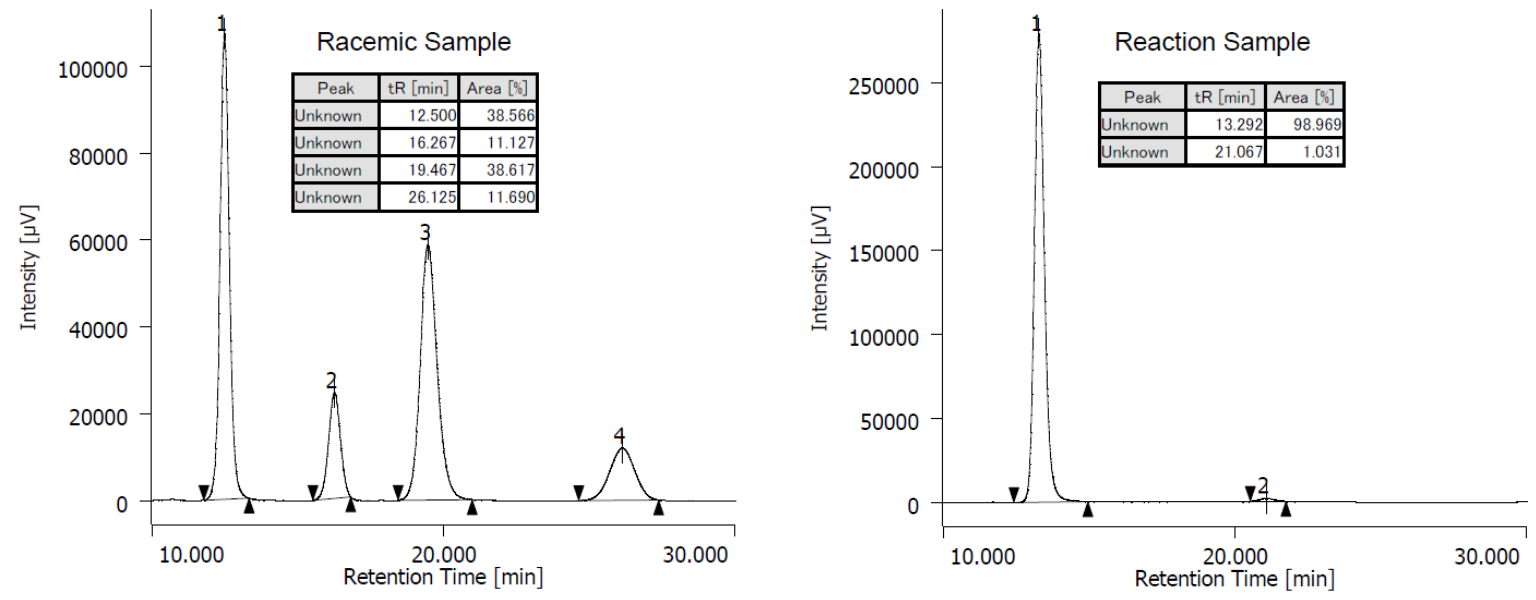

\section{tert-Butyl ((1S,2R)-3-(2,3-dihydro-1H-pyrrolo[2,3-b]pyridin-1-yl)-1-(3-methoxyphenyl)-2-methyl-3-oxopropyl)} carbamate (anti-3c)<smiles>COc1cccc(C(NC(=O)OCc2ccccc2)[C@H](C)C(=O)N2CCc3cccnc32)c1</smiles>

(97\% yield of mixture of diastereomers (anti/syn 12/1), $40 \mathrm{mg}$ ). Anti-diastereomer was separated by PTLC. Colorless oil; ${ }^{1} \mathrm{H}$ NMR $\left(500 \mathrm{MHz}, 323 \mathrm{~K}, \mathrm{CD}_{3} \mathrm{CN}\right): \delta=8.10(\mathrm{~d}, J=5.0$ $\mathrm{Hz}, 1 \mathrm{H}), 7.53(\mathrm{~d}, J=7.0 \mathrm{~Hz}, 1 \mathrm{H}), 7.17(\mathrm{t}, J=7.5 \mathrm{~Hz}, 2 \mathrm{H}), 6.95-6.87(\mathrm{~m}, 3 \mathrm{H}), 6.73(\mathrm{~d}, J=8.0 \mathrm{~Hz}$, $1 \mathrm{H}), 6.63$ (brs, 1H), 4.94 (brs, 1H), 4.72 (brs, 1H), 3.97-3.90 (m, 2H), 3.73 (s, 3H), 3.01-2.86 $(\mathrm{m}, 2 \mathrm{H}), 1.35(\mathrm{~s}, 9 \mathrm{H}), 1.13(\mathrm{~d}, J=5.5 \mathrm{~Hz}, 3 \mathrm{H}) ;{ }^{13} \mathrm{C} \mathrm{NMR}\left(125 \mathrm{MHz}, 323 \mathrm{~K}, \mathrm{CD}_{3} \mathrm{CN}\right): \delta 175.6$, 160.7, 156.9, 156.4, 146.6, 145.4, 135.1, 130.3, 128.3, 119.9, 119.6, 113.6, 113.5, 79.5, 59.4 (br), 56.0, 47.2, 42.7, 28.7, 24.9, 21.1, 16.2; IR (film): $\tilde{v}=3412$ (br), 2974, 2930, 1714, 1646, 1600, 1490, 1421, 1241, 1166, $1048 \mathrm{~cm}^{-1}$; HRMS (ESI) m/z calculated for $\mathrm{C}_{23} \mathrm{H}_{31} \mathrm{O}_{4} \mathrm{~N}_{3}[\mathrm{M}+\mathrm{H}]^{+}:$412.2231, found: 412.2224; $[\alpha]_{D^{25}} 35.0$ (c $0.45, \mathrm{CHCl}_{3}, 98 \%$ ee sample); HPLC analysis CHIRALPAK IA-3 $(\phi 0.46 \mathrm{~cm} \times 25 \mathrm{~cm}), 2$-propanol $/ n$-hexane $=1 / 8$, flow rate $1.0 \mathrm{~mL} / \mathrm{min}$, detection at $254 \mathrm{~nm}, \mathrm{t}=16.1$ 
$\min$ (major), $22.1 \mathrm{~min}$ (minor).
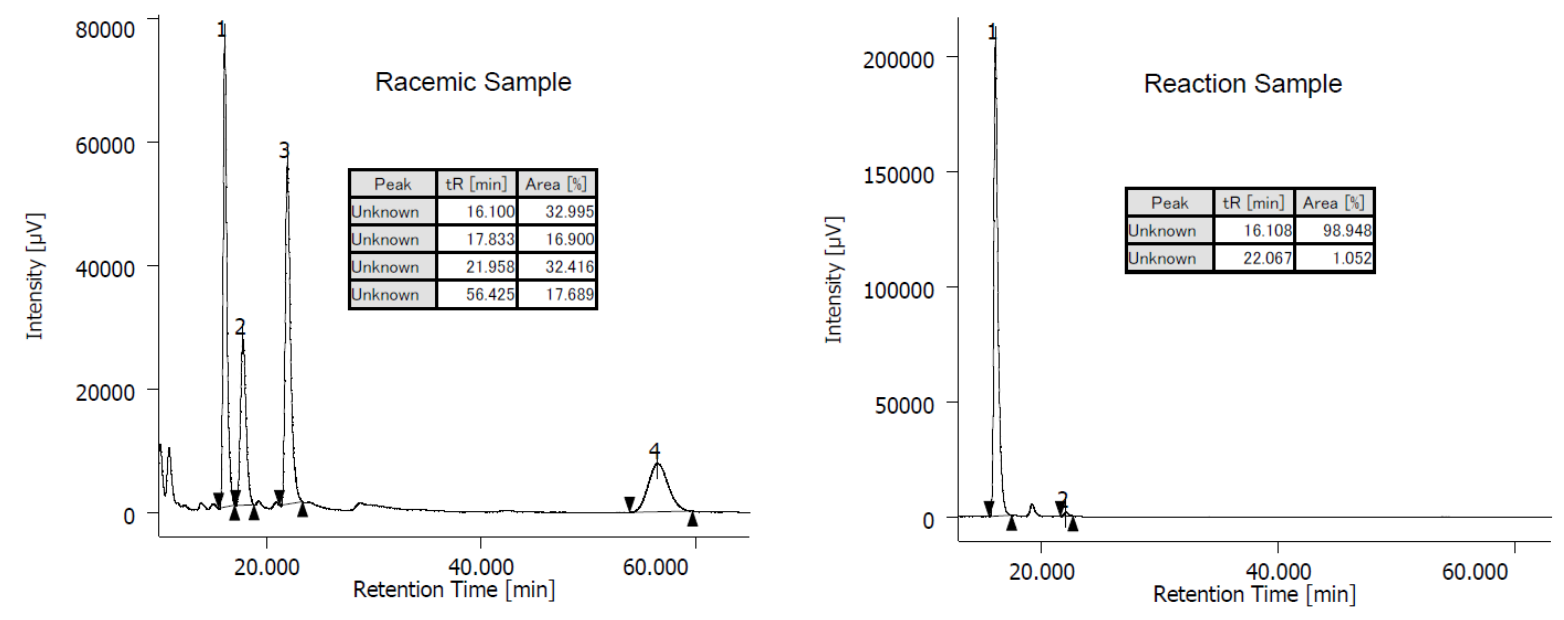

tert-Butyl ((1S,2R)-3-(2,3-dihydro-1H-pyrrolo[2,3-b]pyridin-1-yl)-1-(4-methoxyphenyl)-2-methyl-3-oxopropyl) carbamate (anti-3d)<smiles>COc1ccc(C(NC(=O)OCc2ccccc2)[C@H](C)C(=O)N2CCc3cccnc32)cc1</smiles>

( $82 \%$ yield of mixture of diastereomers (anti/syn >20/1), $34 \mathrm{mg}$ ). Anti-diastereomer was separated by PTLC. Colorless oil; ${ }^{1} \mathrm{H}$ NMR $\left(500 \mathrm{MHz}, 323 \mathrm{~K}, \mathrm{CD}_{3} \mathrm{CN}\right): \delta=8.10(\mathrm{~d}, J=5.0$ $\mathrm{Hz}, 1 \mathrm{H}), 7.53(\mathrm{~d}, J=7.0 \mathrm{~Hz}, 1 \mathrm{H}), 7.22(\mathrm{~d}, J=8.5 \mathrm{~Hz}, 2 \mathrm{H}), 6.94(\mathrm{dd}, J=7.5,5.5 \mathrm{~Hz}, 1 \mathrm{H}), 6.80$ $(\mathrm{d}, J=8.5 \mathrm{~Hz}, 2 \mathrm{H}), 6.58$ (brs, 1H), 4.87 (brs, 1H), 4.69 (brs, 1H), 4.00-3.89 (m, 2H), 3.73 (s, $3 \mathrm{H}), 3.02-2.88(\mathrm{~m}, 2 \mathrm{H}), 1.34(\mathrm{~s}, 9 \mathrm{H}), 1.10(\mathrm{~d}, J=6.5 \mathrm{~Hz}, 3 \mathrm{H}) ;{ }^{13} \mathrm{C}$ NMR $(125 \mathrm{MHz}, 323 \mathrm{~K}$, $\left.\mathrm{CD}_{3} \mathrm{CN}\right): \delta 175.8,160.0,157.0,156.4,146.6,135.8,135.0,128.9,128.3,119.5,114.6,79.4,58.9$ (br), 56.0, 47.2, 43.0, 28.7, 25.0, 16.2; IR (film): $\tilde{v}=3408$ (br), 2973, 2927, 1712, 1644, 1590, 1512, 1420, 1244, 1167, $1035 \mathrm{~cm}^{-1}$; HRMS (ESI) m/z calculated for $\mathrm{C}_{23} \mathrm{H}_{29} \mathrm{O}_{4} \mathrm{~N}_{3} \mathrm{Na}$ [M+Na] $]^{+}$434.2050, found: 434.2048; [ $\left.\alpha\right]_{\mathrm{D}^{25}} 43.1$ (c $0.9, \mathrm{CHCl}_{3}, 97 \%$ ee sample); HPLC analysis CHIRALPAK IF $(\phi 0.46 \mathrm{~cm} \times 25 \mathrm{~cm}), 2$-propanol $/ n$-hexane $=1 / 4$, flow rate $1.0 \mathrm{~mL} / \mathrm{min}$, detection at $254 \mathrm{~nm}$, tr $=17.4$ min (major), $28.8 \mathrm{~min}$ (minor).
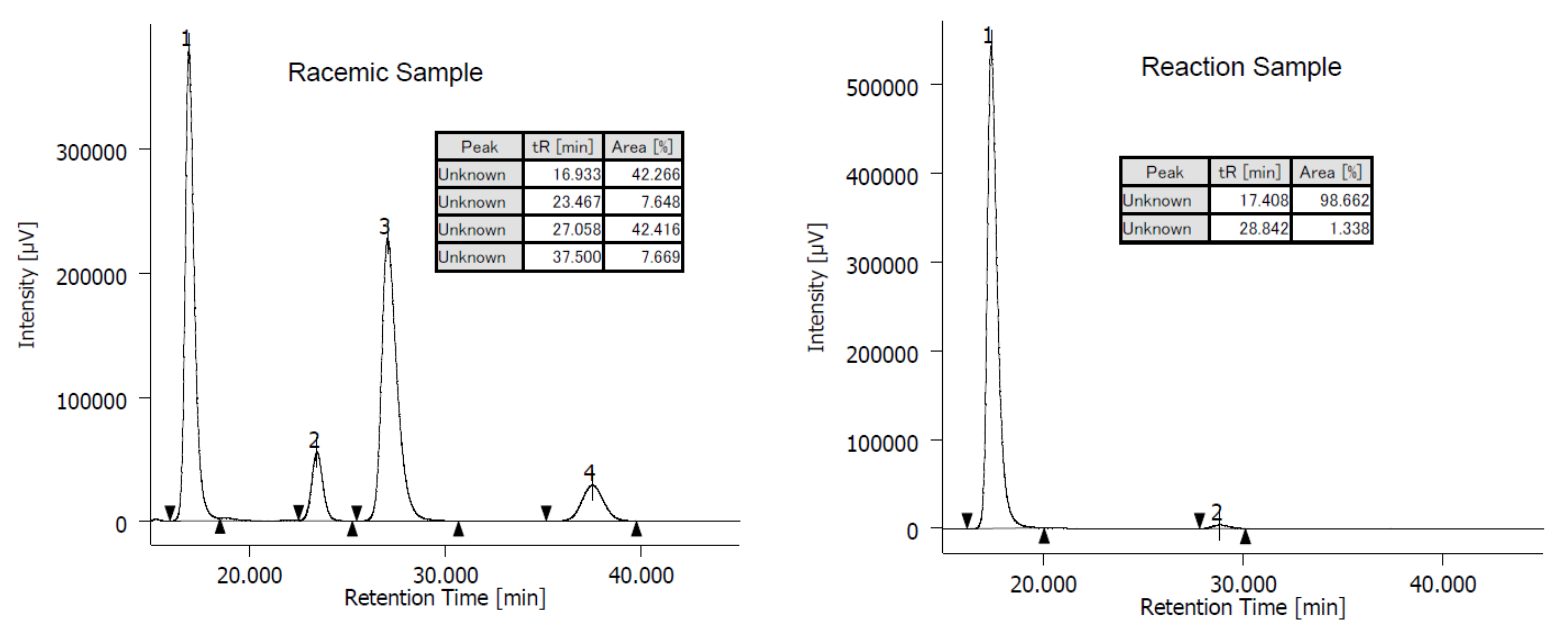

tert-Butyl ((1S,2R)-3-(2,3-dihydro-1H-pyrrolo[2,3-b]pyridine-1-yl)-1-(2-fluorophenyl)-2-methyl-3-oxopropyl) carbamate (anti-3e)

(95\% yield of mixture of diastereomers (anti/syn 14/1), $38 \mathrm{mg}$ ). Anti-diastereomer was separated by PTLC. Colorless oil; ${ }^{1} \mathrm{H}$ NMR $(400 \mathrm{MHz}, 323 \mathrm{~K}, \mathrm{CD} 3 \mathrm{CN}): \delta=8.10(\mathrm{~d}, J=5.0 \mathrm{~Hz}, 1 \mathrm{H}), 7.53(\mathrm{~d}, J=7.0 \mathrm{~Hz}, 1 \mathrm{H}), 7.22(\mathrm{~d}, J=8.5 \mathrm{~Hz}, 2 \mathrm{H}), 6.94(\mathrm{dd}$, $J=7.5,5.5 \mathrm{~Hz}, 1 \mathrm{H}), 6.80(\mathrm{~d}, J=8.5 \mathrm{~Hz}, 2 \mathrm{H}), 6.58$ (brs, 1H), 4.87 (brs, 1H), 4.69 (brs, 1H), 4.00-3.89 (m, 2H), 3.02-2.88 (m, 2H), $1.34(\mathrm{~s}, 9 \mathrm{H}), 1.10$ (d, J=6.5 Hz, 3H); ${ }^{13} \mathrm{C} \mathrm{NMR}\left(100 \mathrm{MHz}, 323 \mathrm{~K}, \mathrm{CD}_{3} \mathrm{CN}\right): \delta$ 175.2, 161.4 (d, J = 242.8 Hz), 156.8, 156.2 , 146.5, 135.1, $130.5(\mathrm{~d}, J=13.1 \mathrm{~Hz}), 130.0(\mathrm{~d}, J=8.1 \mathrm{~Hz}), 129.1(\mathrm{~d}, J=4.4 \mathrm{~Hz}), 128.2,125.3(\mathrm{~d}, J=3.6 \mathrm{~Hz}), 119.6,116.0(\mathrm{~d}, J=$ $21.8 \mathrm{~Hz}), 79.8,53.5$ (br), 47.2, 41.9, 28.7, 24.9, 15.9; $\left.{ }^{19} \mathrm{~F} \mathrm{NMR} \mathrm{(376} \mathrm{MHz,} 323 \mathrm{~K}, \mathrm{CD}_{3} \mathrm{CN}\right): \delta=-120.4$ (s, 1F); IR (film): $\widetilde{v}=$ 3403 (br), 2977, 1715, 1653, 1421, 1169, $1002 \mathrm{~cm}^{-1}$; HRMS (ESI) $\mathrm{m} / z$ calculated for $\mathrm{C}_{22} \mathrm{H}_{27} \mathrm{O}_{3} \mathrm{~N}_{3} \mathrm{~F}$ [M+H]+: 400.2031, found: 


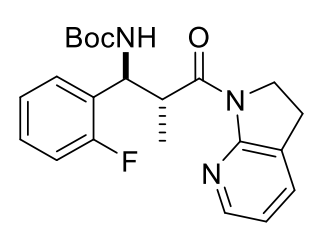
400.2023; [ $\alpha$ ] $\mathrm{D}^{26} 37.7$ ( c 1.0, $\mathrm{CHCl}_{3}, 95 \%$ ee sample); HPLC analysis CHIRALPAK IF $(\phi 0.46 \mathrm{~cm} x$ $25 \mathrm{~cm}), 2$-propanol $/ n$-hexane $=1 / 4$, flow rate $1.0 \mathrm{~mL} / \mathrm{min}$, detection at $254 \mathrm{~nm}$, $\mathrm{t}=12.5 \mathrm{~min}$ (major), $15.5 \mathrm{~min}$ (minor).
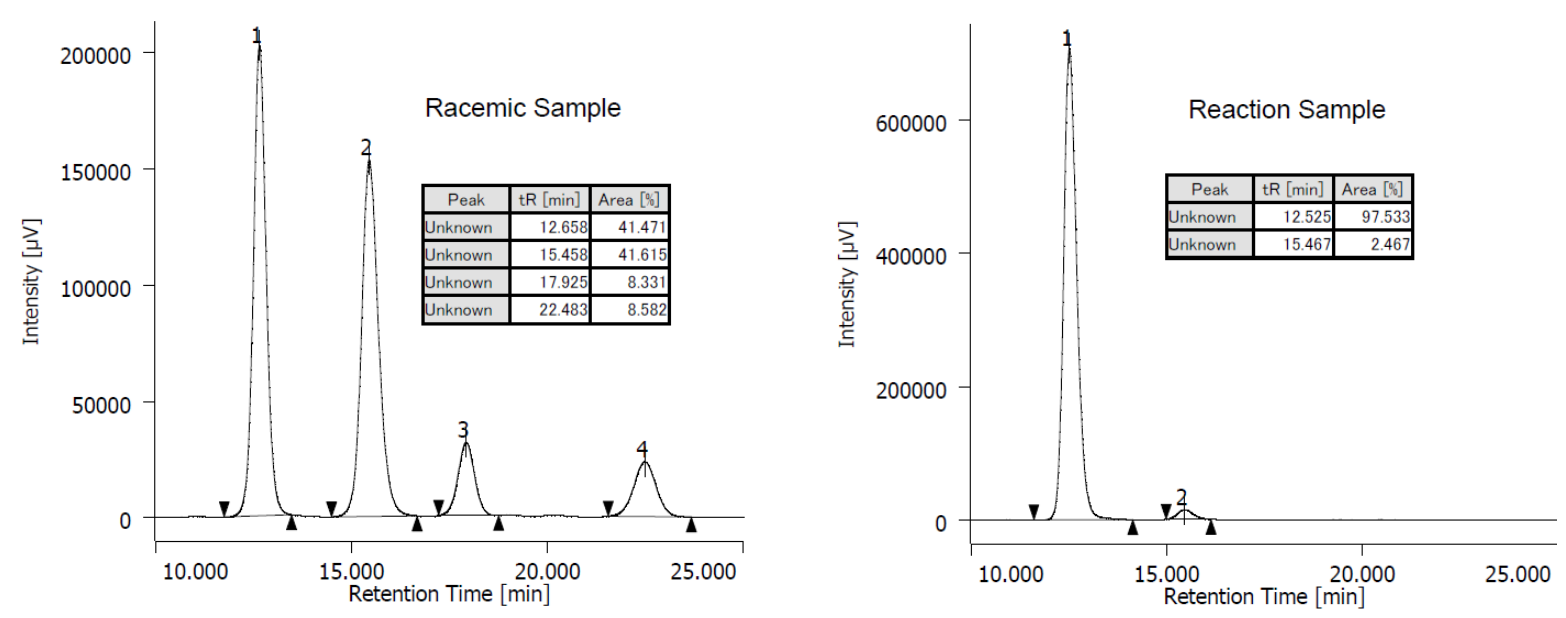

tert-Butyl ((1S,2R)-3-(2,3-dihydro-1H-pyrrolo[2,3-b]pyridin-1-yl)-1-(3-fluorophenyl)-2-methyl-3-oxopropyl) carbamate (anti-3f)<smiles>C[C@H](C(=O)N1CCc2cccnc21)C(NC(=O)[O-])c1cccc(F)c1</smiles>

(95\% yield of mixture of diastereomers (anti/syn 10/1), $38 \mathrm{mg}$ ). Anti-diastereomer was separated by PTLC. Colorless oil; ${ }^{1} \mathrm{H}$ NMR (400 MHz, $\left.323 \mathrm{~K}, \mathrm{CD}_{3} \mathrm{CN}\right): \delta=8.10(\mathrm{~d}, J=5.2 \mathrm{~Hz}$, $1 \mathrm{H}), 7.53(\mathrm{~d}, J=7.6 \mathrm{~Hz}, 1 \mathrm{H}), 7.30-7.24(\mathrm{~m}, 1 \mathrm{H}), 7.14(\mathrm{~d}, J=7.6 \mathrm{~Hz}, 1 \mathrm{H}), 7.07(\mathrm{~d}, J=10.4 \mathrm{~Hz}, 1 \mathrm{H})$, 6.96-6.88 (m, 2H), 6.67 (brs, 1H), 4.93 (brs, 1H), 4.77 (brs, 1H), 4.01-3.87 (m, 2H), 3.03-2.86 (m, 2H), $1.35(\mathrm{~s}, 9 \mathrm{H}), 1.14(\mathrm{~d}, J=6.8 \mathrm{~Hz}, 3 \mathrm{H}) ;{ }^{13} \mathrm{C} \mathrm{NMR}\left(100 \mathrm{MHz}, 323 \mathrm{~K}, \mathrm{CD}{ }_{3} \mathrm{CN}\right): \delta$ 175.4, 163.8 (d, $J=242.0 \mathrm{~Hz}), 156.8,156.4,146.7,135.1,131.0(\mathrm{~d}, J=8.0 \mathrm{~Hz}), 128.3,123.7,119.6,114.7(\mathrm{~d}, J=21.1 \mathrm{~Hz}), 114.5(\mathrm{~d}, J=21.8 \mathrm{~Hz})$, 79.7, 58.9 (br), 47.2, 42.7, 28.7, 24.9, 16.1; $\left.{ }^{19} \mathrm{~F} \mathrm{NMR} \mathrm{(376} \mathrm{MHz,} 323 \mathrm{~K}, \mathrm{CD}_{3} \mathrm{CN}\right): \delta=-115.2$ (s, 1F); IR (film): $\widetilde{v}=3402$ (br), 2976, 2929, 1713, 1645, 1590, 1489, 1421, 1241, $1002 \mathrm{~cm}^{-1}$; HRMS (ESI) m/z calculated for $\mathrm{C}_{22} \mathrm{H}_{26} \mathrm{O}_{3} \mathrm{~N}_{3} \mathrm{FNa}[\mathrm{M}+\mathrm{Na}]^{+}$: 422.1850, found: 422.1847; $[\alpha]^{26} 52.6$ (c $0.30, \mathrm{CHCl}_{3}, 97 \%$ ee sample); HPLC analysis CHIRALPAK IF ( $\phi 0.46 \mathrm{~cm} \times 25$ $\mathrm{cm}$ ), 2-propanol $/ n$-hexane $=1 / 4$, flow rate $1.0 \mathrm{~mL} / \mathrm{min}$, detection at $254 \mathrm{~nm}, \mathrm{t}_{\mathrm{R}}=10.9 \mathrm{~min}$ (major), $18.3 \mathrm{~min}$ (minor).
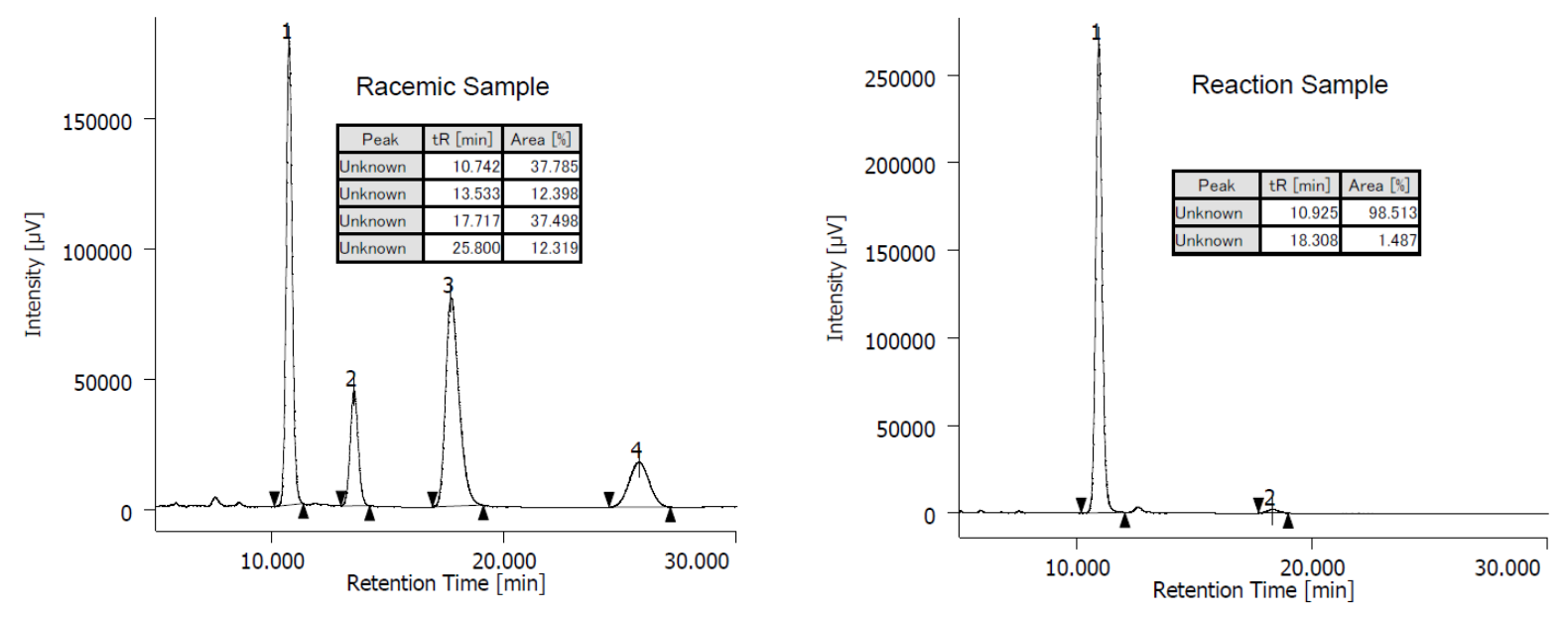

\section{tert-Butyl ((1S,2R)-3-(2,3-dihydro-1H-pyrrolo[2,3-b]pyridin-1-yl)-1-(4-fluorophenyl)-2-methyl-3-oxopropyl)}

\section{carbamate (anti-3g)}<smiles>C[C@H](C(=O)N1CCc2cccnc21)[C@H](NC(=O)[O-])c1ccc(F)cc1</smiles>

(Amide 1a (1 g, $5.67 \mathrm{mmol})$ and imine $2 \mathrm{~g}(2.53 \mathrm{~g}, 11.34 \mathrm{mmol})$ were used. $2.112 \mathrm{~g}$ of product (93\% yield) was obtained as mixture of diastereomers (anti/syn $=12 / 1,95 \%$ ee (anti)). Anti-diastereomer was separated by $\mathrm{SiO}_{2}$ column chromatography (hexane/acetone, 5:1). White solid, m.p.: $96-98{ }^{\circ} \mathrm{C}$; ${ }^{1} \mathrm{H}$ NMR $\left(400 \mathrm{MHz}, 323 \mathrm{~K}, \mathrm{CD}_{3} \mathrm{CN}\right): \delta=8.10(\mathrm{~d}, J=6.4 \mathrm{~Hz}, 1 \mathrm{H})$, 7.54-7.52 (m, 1H), 7.34-7.31 (m, 2H), 7.01-6.93 (m, 3H), 6.64 (brs, 1H), 4.90 (brs, 1H), 4.75 (brs, 

$1 \mathrm{H}), 4.01-3.88(\mathrm{~m}, 2 \mathrm{H}), 3.03-2.87(\mathrm{~m}, 2 \mathrm{H}), 1.34(\mathrm{~s}, 9 \mathrm{H}), 1.12(\mathrm{~d}, J=6.8 \mathrm{~Hz}, 3 \mathrm{H}) ;{ }^{13} \mathrm{C} \mathrm{NMR}(100 \mathrm{MHz}, 323 \mathrm{~K}, \mathrm{CD} 3 \mathrm{CN}): \delta$ $175.5,162.9(\mathrm{~d}, J=241.3 \mathrm{~Hz}), 156.9,156.4,146.6,139.8,135.1,129.6(\mathrm{~d}, J=8.0 \mathrm{~Hz}), 128.3,119.6,115.8(\mathrm{~d}, J=21.2 \mathrm{~Hz}), 79.6$, 58.8 (br), 47.3, 42.8, 28.7, 24.9, 16.1; ${ }^{19} \mathrm{~F}$ NMR (376 MHz, $323 \mathrm{~K}, \mathrm{CD}_{3} \mathrm{CN}$ ): $\delta=-117.8$ (s, 1F); IR (film): $\tilde{v}=3405$ (br), 2976 , $2929,1712,1644,1591,1509,1422,1161 \mathrm{~cm}^{-1}$; HRMS (ESI) $\mathrm{m} / z$ calculated for $\mathrm{C}_{22} \mathrm{H}_{26} \mathrm{O}_{3} \mathrm{~N}_{3} \mathrm{FNa}[\mathrm{M}+\mathrm{Na}]^{+}: 422.1850$, found: 422.1844; $[\alpha]_{\mathrm{D}^{25}} 55.9$ (c 1.0, $\mathrm{CHCl}_{3}, 95 \%$ ee sample); HPLC analysis CHIRALPAK IF ( $\left.\phi 0.46 \mathrm{~cm} \mathrm{x} 25 \mathrm{~cm}\right)$, 2-propanol $/ n$-hexane $=1 / 4$, flow rate $1.0 \mathrm{~mL} / \mathrm{min}$, detection at $254 \mathrm{~nm}, \mathrm{t}=11.3 \mathrm{~min}$ (major), $17.8 \mathrm{~min}$ (minor).
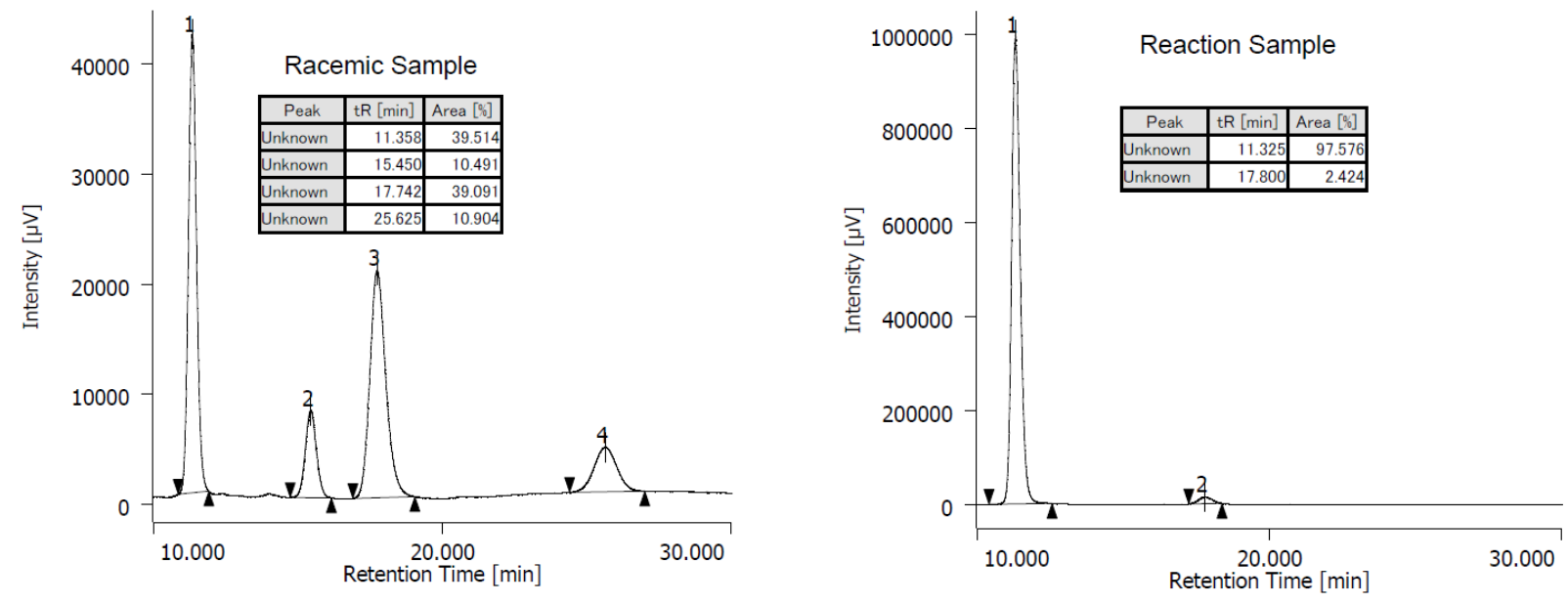

tert-Butyl ((1S,2R)-3-(2,3-dihydro-1H-pyrrolo[2,3-b]pyridin-1-yl)-2-methyl-1-(3-nitrophenyl)-3-oxopropyl)carbamate (anti-3h)<smiles>C[C@H](NC(=O)OCc1cccc([N+](=O)[O-])c1)C(=O)N1CCc2cccnc21</smiles>

(66\% yield of mixture of diastereomers (anti/syn 6.8/1), $28 \mathrm{mg}$ ). Anti-diastereomer was separated by PTLC. White solid, m.p.: 131-132 ${ }^{\circ} \mathrm{C} ;{ }^{1} \mathrm{H}$ NMR $\left(400 \mathrm{MHz}, 323 \mathrm{~K}, \mathrm{CD}_{3} \mathrm{CN}\right): \delta=$ 8.18-8.17 (m, 1H), $8.07(\mathrm{~d}, J=5.2 \mathrm{~Hz}, 1 \mathrm{H}), 7.98(\mathrm{~d}, J=8.4 \mathrm{~Hz}, 1 \mathrm{H}), 7.71(J=8.0 \mathrm{~Hz}, 1 \mathrm{H}) 7.51-$ $7.46(\mathrm{~m}, 2 \mathrm{H}), 6.92$ (dd, J = 7.6, $5.2 \mathrm{~Hz}, 1 \mathrm{H}), 6.73$ (brs, 1H), 5.05 (brs, 1H), 4.92 (brs, 1H), 3.99$3.86(\mathrm{~m}, 2 \mathrm{H}), 3.01-2.81(\mathrm{~m}, 2 \mathrm{H}), 1.37(\mathrm{~s}, 9 \mathrm{H}), 1.20(\mathrm{~d}, J=6.4 \mathrm{~Hz}, 3 \mathrm{H}) ;{ }^{13} \mathrm{C} \mathrm{NMR}(100 \mathrm{MHz}, 323$ $\left.\mathrm{K}, \mathrm{CD}_{3} \mathrm{CN}\right): \delta 175.0,156.6,156.5,149.4,146.6,146.0,135.1,134.1,130.4,128.2,123.0,122.7,119.7,79.9,58.7$ (br), $47.1,42.4$, 28.7, 24.9, 16.0; IR (film): $\widetilde{v}=3400$ (br), 2977, 2917, 1712, 1645, 1530, 1421, 1350, 1165, 1002 cm-1; HRMS (ESI) m/z calculated for $\mathrm{C}_{22} \mathrm{H}_{27} \mathrm{O}_{5} \mathrm{~N}_{4}[\mathrm{M}+\mathrm{H}]^{+}: 427.1976$, found: 427.1965; [ $\left.\alpha\right]_{\mathrm{D}}^{26} 37.6$ (c 0.70, $\mathrm{CHCl}_{3}, 92 \%$ ee sample); HPLC analysis CHIRALPAK IA $(\phi 0.46 \mathrm{~cm} \times 25 \mathrm{~cm}), 2$-propanol $/ n$-hexane $=1 / 4$, flow rate $1.0 \mathrm{~mL} / \mathrm{min}$, detection at $254 \mathrm{~nm}$, tR $=12.2$ $\min$ (major), $15.0 \mathrm{~min}$ (minor).
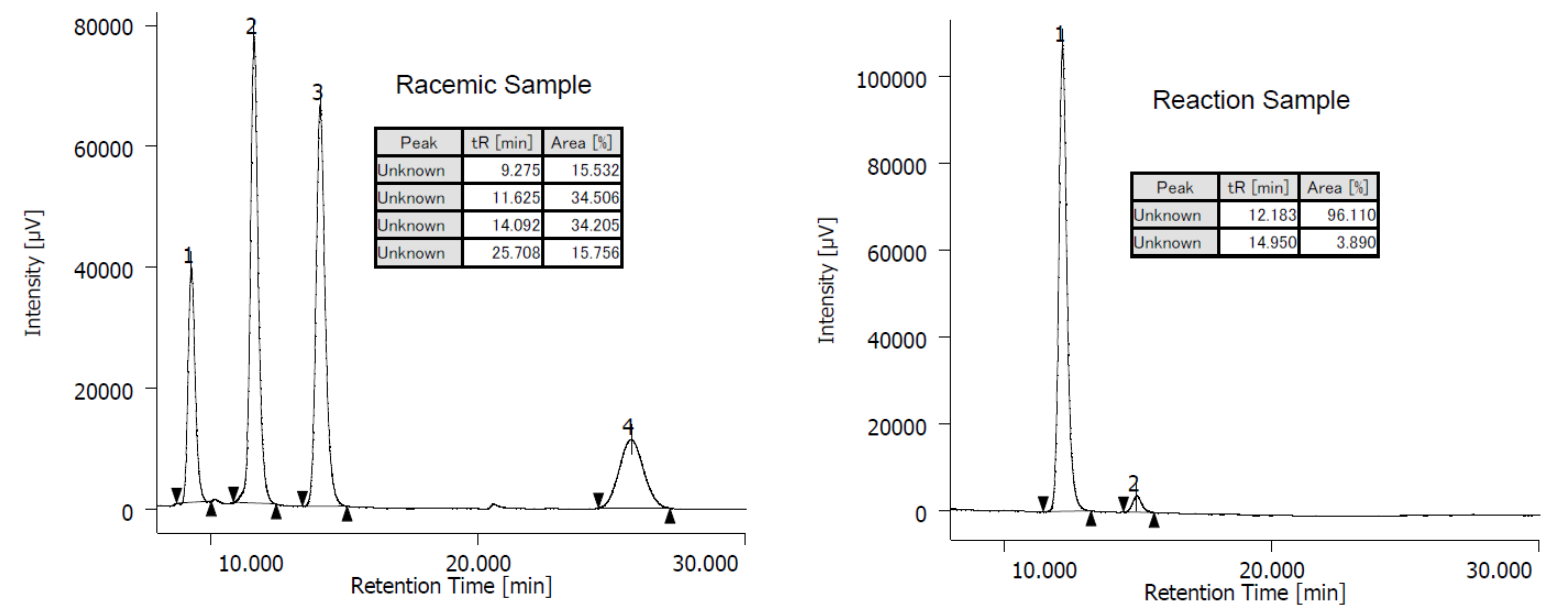

\section{tert-Butyl ((1S,2R)-1-(3-bromophenyl)-3-(2,3-dihydro-1H-pyrrolo[2,3-b]pyridin-1-yl)-2-methyl-3-oxopropyl)} carbamate (anti-3i)<smiles>C[C@H](NC(=O)OCc1ccccc1)C(=O)N1CCc2cccnc21</smiles>
(95\% yield of mixture of diastereomers (anti/syn 10/1), $44 \mathrm{mg}$ ). Anti-diastereomer was separated by PTLC. Colorless oil; ${ }^{1} \mathrm{H}$ NMR (500 MHz, $\left.323 \mathrm{~K}, \mathrm{CD}_{3} \mathrm{CN}\right): \delta=8.09$ (d, J=4.5 Hz, 

$1 \mathrm{H}), 7.53(\mathrm{~d}, J=7.0 \mathrm{~Hz} 1 \mathrm{H}), 7.48$ (brs, 1H), 7.32-7.28 (m, 2H), 7.18-7.15 (m, 1H), 6.95-6.93 (m, 1H), 6.64 (brs, 1H), 4.95 (brs, 1H), 4.75 (brs, 1H), 3.99-3.88 (m, 2H), 3.01-2.87 (m, 2H), $1.36(\mathrm{~s}, 9 \mathrm{H}), 1.15$ (d, J = 6.0 Hz, 3H); ${ }^{13} \mathrm{C}$ NMR $(125 \mathrm{MHz}$, $\left.323 \mathrm{~K}, \mathrm{CD}_{3} \mathrm{CN}\right): \delta$ 175.3, 156.6, 156.3, 146.6, 146.5, 135.1,131.1, 131.0, 130.8, 128.2, 126.7, 122.8, 119.7, 79.8, 58.9 (br), 47.1, 42.5, 28.7, 24.9, 16.1; IR (film): $\widetilde{v}=3390$ (br), 2973, 2923, 2851, 1713, 1645, 1591, 1421, 1241, 1165, $1000 \mathrm{~cm}^{-1}$; HRMS (ESI) $m / z$ calculated for $\mathrm{C}_{22} \mathrm{H}_{26} \mathrm{O}_{3} \mathrm{~N}_{3} \mathrm{BrNa}[\mathrm{M}+\mathrm{Na}]^{+}: 482.1050$, found: 482.1041; [ $\left.\alpha\right]_{\mathrm{D}^{26}} 41.4$ (c $0.70, \mathrm{CHCl}_{3}, 95 \%$ ee sample); HPLC analysis CHIRALPAK IA-3 $(\phi 0.46 \mathrm{~cm} \times 25 \mathrm{~cm}), 2$-propanol $/ n$-hexane $=1 / 8$, flow rate $1.0 \mathrm{~mL} / \mathrm{min}$, detection at $254 \mathrm{~nm}, \mathrm{t}_{\mathrm{R}}=12.6 \mathrm{~min}$ (major), $13.9 \mathrm{~min}$ (minor).
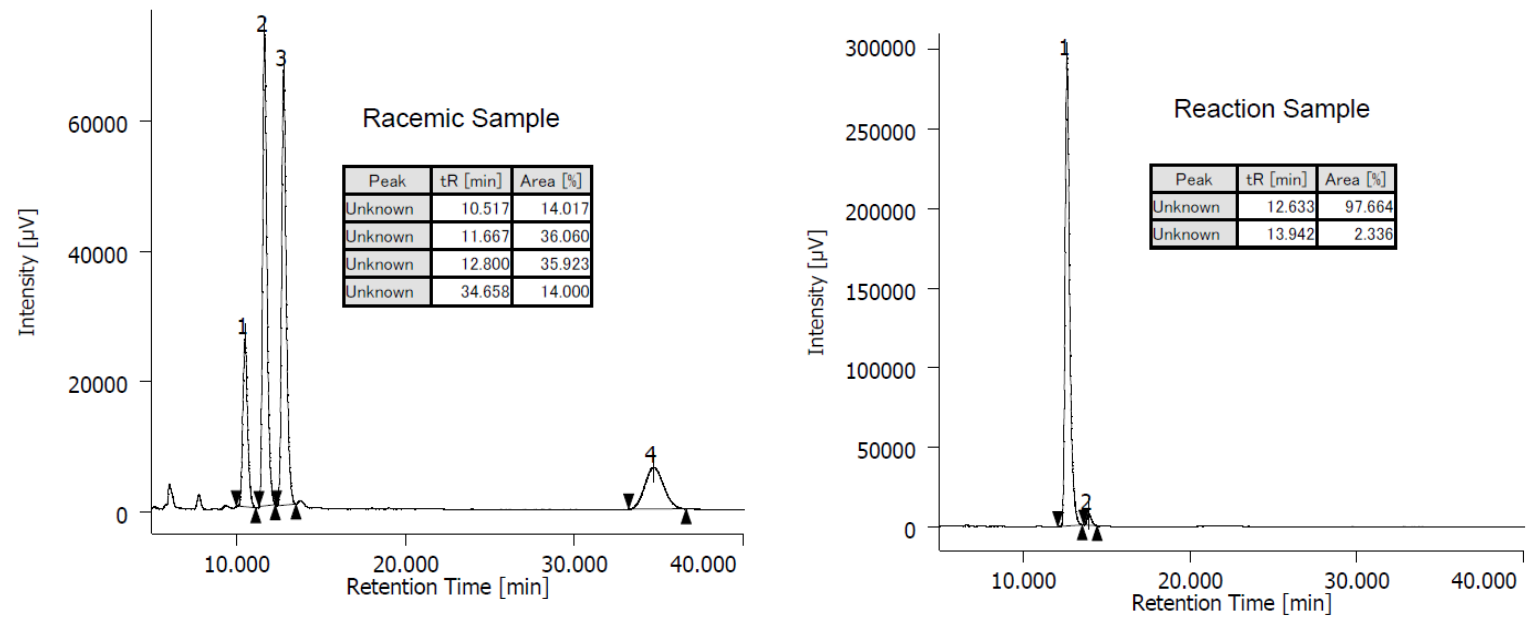

4-((1S,2R)-1-((tert-Butoxycarbonyl)amino)-3-(2,3-dihydro-1H-pyrrolo[2,3-b]pyridin-1-yl)-2-methyl-3-oxopropyl)phenyltrifluoromethanesulfonate (anti-3j)<smiles>CC(C(=O)N1CCc2cccnc21)[C@H](NC(=O)c1ccccc1)c1ccccc1</smiles>

( $90 \%$ yield of mixture of diastereomers (anti/syn 13/1), $48 \mathrm{mg}$ ). Anti-diastereomer was separated by PTLC. Colorless oil; ${ }^{1} \mathrm{H}$ NMR $\left(400 \mathrm{MHz}, 323 \mathrm{~K}, \mathrm{CD}_{3} \mathrm{CN}\right): \delta=8.08(\mathrm{~d}, J=5.6 \mathrm{~Hz}$, $1 \mathrm{H}), 7.53-7.51(\mathrm{~m}, 1 \mathrm{H}), 7.46(J=8.8 \mathrm{~Hz}, 2 \mathrm{H}), 7.24(J=8.4 \mathrm{~Hz}, 2 \mathrm{H}), 6.94(\mathrm{dd}, J=7.6,5.2 \mathrm{~Hz}$, $1 \mathrm{H}), 6.68(\mathrm{brs}, 1 \mathrm{H}), 4.97$ (brs, $1 \mathrm{H}), 4.82(\mathrm{brs}, 1 \mathrm{H}), 4.00-3.86(\mathrm{~m}, 2 \mathrm{H}), 3.01-2.84(\mathrm{~m}, 2 \mathrm{H}), 1.35(\mathrm{~s}$, 9H), $1.16(\mathrm{~d}, J=6.8 \mathrm{~Hz}, 3 \mathrm{H}) ;{ }^{13} \mathrm{C}$ NMR $(100 \mathrm{MHz}, 323 \mathrm{~K}, \mathrm{CD} 3 \mathrm{CN}): \delta$ 175.2, 156.7, 156.4, 149.6, 146.6, 144.5, 135.1, 129.8, 128.2, 122.1, 119.9 (q, $J=322.3 \mathrm{~Hz}), 119.7,79.9,58.8$ (br), 47.1, 42.5, 28.7, 24.9, 16.1; ${ }^{19} \mathrm{~F}$ NMR (376 $\mathrm{MHz}, 323 \mathrm{~K}, \mathrm{CD}_{3} \mathrm{CN}$ ): $\delta=-74.0$ (s, 3F); IR (film): $\widetilde{v}=3403$ (br), 2977, 2930, 1713, 1643, 1498, 1421, 1212, 1141, $888 \mathrm{~cm}^{-1}$; HRMS (ESI) $\mathrm{m} / \mathrm{z}$ calculated for $\mathrm{C}_{23} \mathrm{H}_{27} \mathrm{O}_{6} \mathrm{~N}_{3} \mathrm{~F}_{3} \mathrm{~S}[\mathrm{M}+\mathrm{H}]^{+}: 530.1567$, found: 530.1559; $[\alpha]_{\mathrm{D}^{26}} 60.9$ (c 0.55, $\mathrm{CHCl}_{3}, 96 \%$ ee sample); HPLC analysis CHIRALPAK IF $(\phi 0.46 \mathrm{~cm} \times 25 \mathrm{~cm}), 2$-propanol $/ \mathrm{n}$-hexane $=1 / 4$, flow rate $1.0 \mathrm{~mL} / \mathrm{min}$, detection at $254 \mathrm{~nm}, \mathrm{tR}=9.8 \mathrm{~min}$ (major), $13.2 \mathrm{~min}(\mathrm{~min})$.
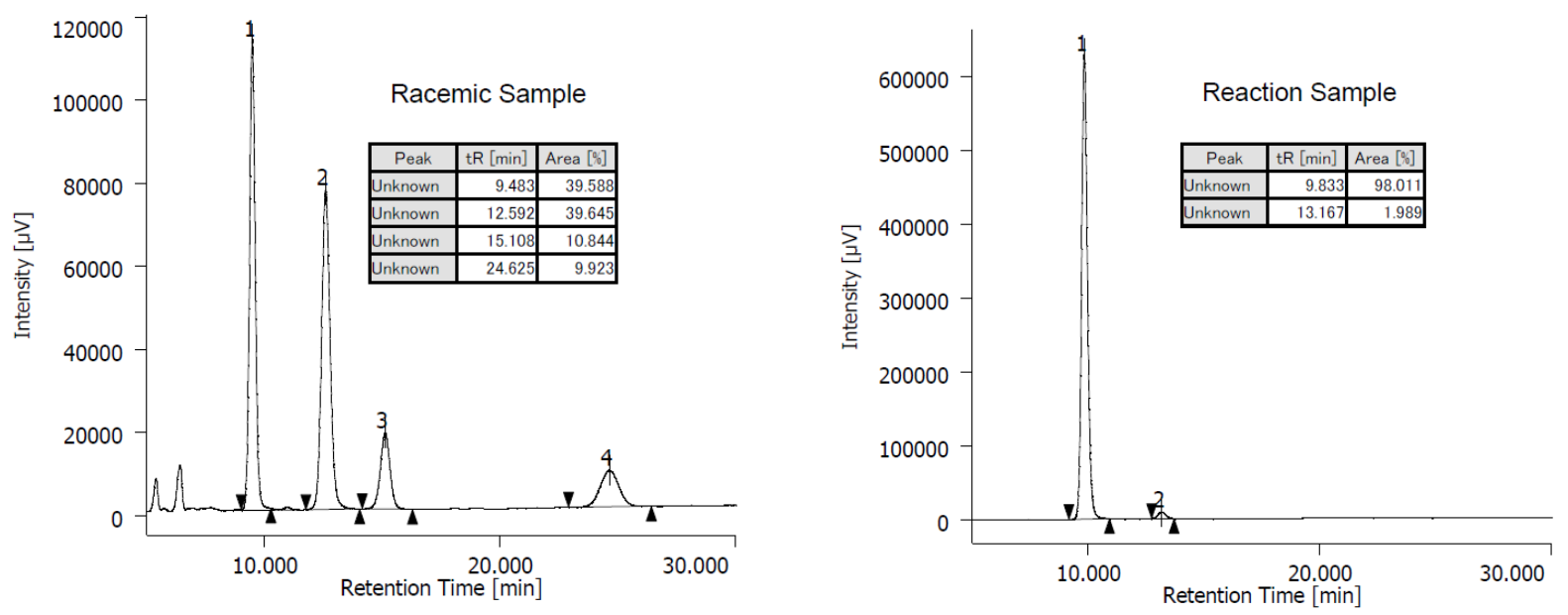

\section{tert-Butyl}

((1S,2R)-3-(2,3-dihydro-1H-pyrrolo[2,3-b]pyridin-1-yl)-2-methyl-3-oxo-1-(3-(4,4,5,5-tetramethyl-1,3,2-dioxa-borolan-2 -yl)phenyl)propyl)carbamate (anti-3k) 
<smiles>C[C@H](C(=O)N1CCc2cccnc21)[C@H](NC(=O)OCc1ccccc1)c1cccc([Pb]Cc2ccccc2)c1</smiles>
(45\% yield of mixture of diastereomers (anti/syn 9.5/1), $23 \mathrm{mg}$ ). Anti-diastereomer was separated by PTLC. Colorless oil; ${ }^{1} \mathrm{H}$ NMR (500 MHz, $\left.323 \mathrm{~K}, \mathrm{CD}_{3} \mathrm{CN}\right): \delta=8.08(\mathrm{~d}, J=4.5$ $\mathrm{Hz}, 1 \mathrm{H}), 7.66$ (brs, 1H), 7.51-7.48 (m, 2H), 7.40 (d, J= 7.5 Hz, 1H), 7.26-7.23 (m, 1H), 6.946.91 (m, 1H), 6.63 (brs, 1H), 4.96 (brs, 1H), 4.75 (brs, 1H), 3.96-3.89 (m, 2H), 2.99-2.82 (m, 2H), 1.34 (brs, 21H), 1.16 (brs, 3H); $\left.{ }^{13} \mathrm{C} \mathrm{NMR} \mathrm{(125} \mathrm{MHz,} 323 \mathrm{~K}, \mathrm{CD}_{3} \mathrm{CN}\right): \delta$ 175.6, 156.8, 156.5, 146.5, 143.0, 135.0, 134.1, 133.7, 130.7, 129.2, 128.7, 128.2, 119.6, 85.0, 79.5, 59.3 (br), 47.1, 42.6, 28.7, 25.4, 24.9, 16.1; ${ }^{11} \mathrm{~B}$ NMR (128 MHz, $\left.300 \mathrm{~K}, \mathrm{CD}_{3} \mathrm{CN}\right): \delta=33.2$ (br); IR (film): $\widetilde{v}=3410$ (br), 2977, 2932, 1715, 1646, 1489, 1421, 1362, 1166, $1145,854 \mathrm{~cm}^{-1}$; HRMS (ESI) $\mathrm{m} / z$ calculated for $\mathrm{C}_{28} \mathrm{H}_{38} \mathrm{O}_{5} \mathrm{~N}_{3} \mathrm{BNa}[\mathrm{M}+\mathrm{Na}]^{+}:$530.2797, found: $530.2801 ;[\alpha]^{25} 49.9(c 0.07$, $\mathrm{CHCl}_{3}, 91 \%$ ee sample); HPLC analysis CHIRALPAK IF ( $\left.\phi 0.46 \mathrm{~cm} \times 25 \mathrm{~cm}\right), 2$-propanol $/ n$-hexane $=1 / 4$, flow rate 1.0 $\mathrm{mL} / \mathrm{min}$, detection at $254 \mathrm{~nm}, \mathrm{t}_{\mathrm{R}}=8.7 \mathrm{~min}$ (major), $12.1 \mathrm{~min}$ (minor). The aromatic sp ${ }^{2}$ carbon next to the boron atom was not clearly observed due to quadruple relaxation of the boron nucleus and tentatively assigned as 129.2 ppm. (There was partial degradation during isolation of anti-diastereomer by PTLC).
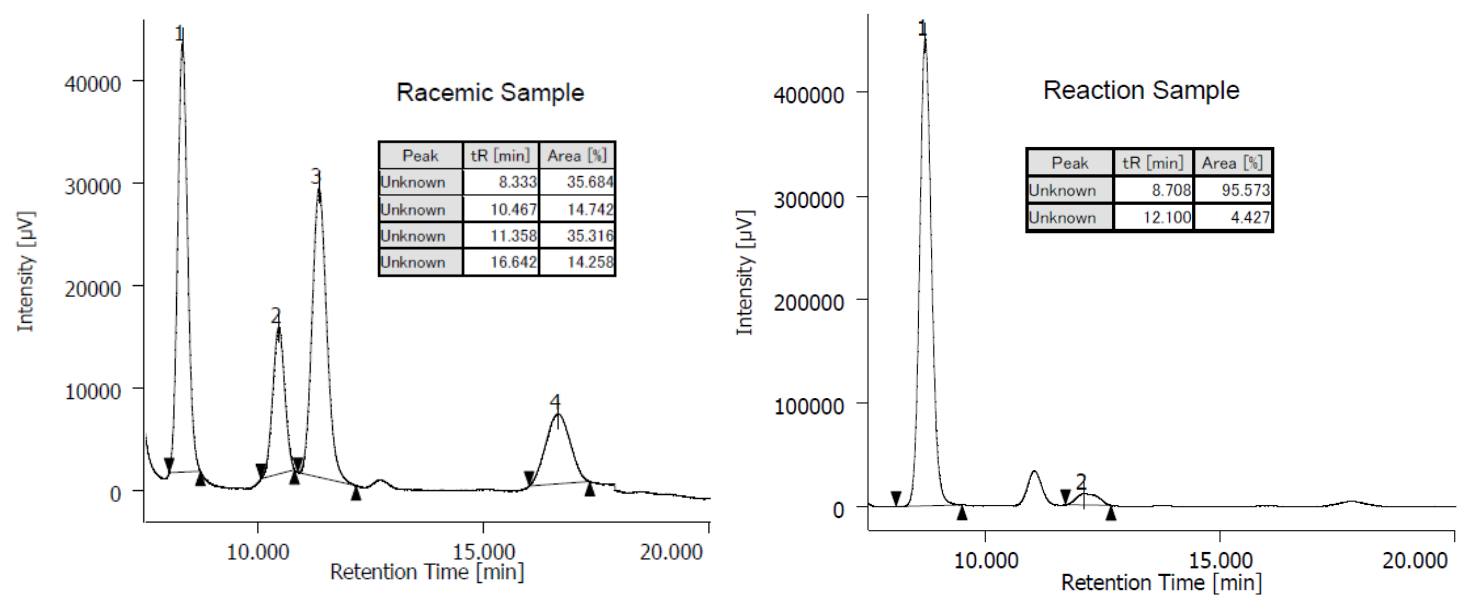

\section{tert-Butyl ((1S,2R)-3-(2,3-dihydro-1H-pyrrolo[2,3-b]pyridin-1-yl)-1-(furan-3-yl)-2-methyl-3-oxopropyl)carbamate (anti-31)}<smiles>C[C@H](C(=O)N1CCc2cccnc21)[C@H](NC(=O)O)c1ccoc1</smiles>

(78\% yield of mixture of diastereomers (anti/syn 9.3/1), $29 \mathrm{mg}$ ). Anti-diastereomer was separated by PTLC. Colorless oil; ${ }^{1} \mathrm{H}$ NMR (500 MHz, $\left.323 \mathrm{~K}, \mathrm{CD}_{3} \mathrm{CN}\right): \delta=8.12$ (d, J=5.0 Hz, 1H), $7.57(J=9.0$ $\mathrm{Hz}, 1 \mathrm{H}), 7.35$ (brs, 2H), 6.96 (dd, $J=7.0,5.0 \mathrm{~Hz}, 1 \mathrm{H}), 6.42$ (brs, 2H), 4.87 (brs, 1H), 4.76 (brs, 1H), 4.03-3.91 (m, 2H), 3.06-2.96 (m, 2H), 1.39 (s, 9H), $1.18(\mathrm{~d}, J=6.8 \mathrm{~Hz}, 3 \mathrm{H}) ;{ }^{13} \mathrm{C}$ NMR $(125 \mathrm{MHz}, 323 \mathrm{~K}$, $\left.\mathrm{CD}_{3} \mathrm{CN}\right): \delta 175.9,156.9,156.5,146.9,144.3,140.6,135.1,128.5,128.3,119.6,110.3,79.5,51.6$ (br), 47.1, 42.3, 28.7, 24.9, 16.0; IR (film): $\tilde{v}=3412$ (br), 2975, 2926, 1711, 1646, 1590, 1491, 1420, 1307, 1165, 785 cm-1; HRMS (ESI) $m / z$ calculated for $\mathrm{C}_{20} \mathrm{H}_{25} \mathrm{O}_{4} \mathrm{~N}_{3} \mathrm{Na}[\mathrm{M}+\mathrm{Na}]^{+}$: 394.1737, found: 394.1729; [ $\left.\alpha\right]_{\mathrm{D}^{26}} 42.0$ (c 0.55, CHCl3, 94\% ee sample); HPLC analysis CHIRALPAK IF $(\phi 0.46 \mathrm{~cm} \times 25 \mathrm{~cm}), 2$-propanol $/ n$-hexane $=1 / 4$, flow rate $1.0 \mathrm{~mL} / \mathrm{min}$, detection at $254 \mathrm{~nm}$, tR $=11.1 \mathrm{~min}$ (major), $22.9 \mathrm{~min}$ (minor).
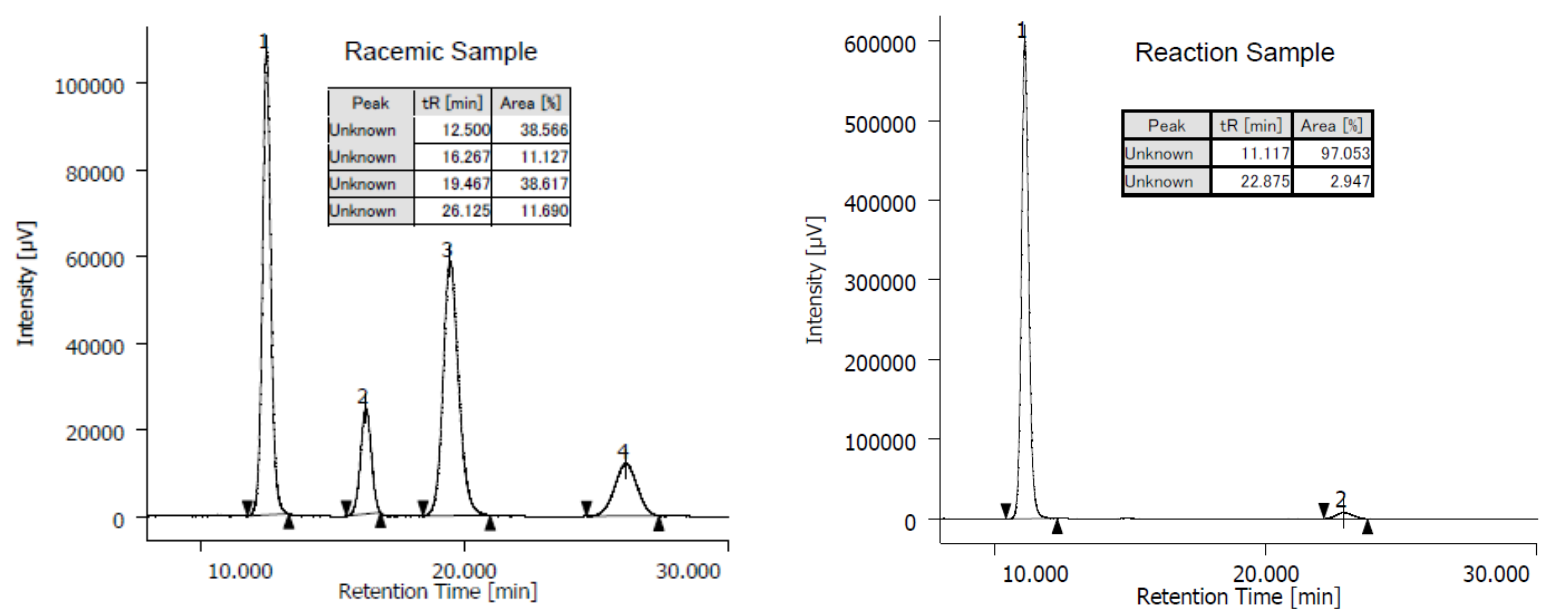
tert-Butyl ((1S,2R)-3-(2,3-dihydro-1H-pyrrolo[2,3-b]pyridin-1-yl)-2-methyl-3-oxo-1-(thiophen-2-yl)propyl)carbamate (anti-3m)<smiles>C[C@H](C(=O)N1CCc2cccnc21)c1cccs1</smiles>

( $90 \%$ yield of mixture of diastereomers (anti/syn 12/1), $35 \mathrm{mg}$ ). Anti-diastereomer was separated by PTLC. Colorless oil; ${ }^{1} \mathrm{H}$ NMR $\left(500 \mathrm{MHz}, 323 \mathrm{~K}, \mathrm{CD}_{3} \mathrm{CN}\right): \delta=8.12(\mathrm{~d}, J=6.4 \mathrm{~Hz}, 1 \mathrm{H}), 7.58-7.56$ $(\mathrm{m}, 1 \mathrm{H}), 7.20(\mathrm{dd}, J=4.8,1.2 \mathrm{~Hz}, 1 \mathrm{H}), 6.97(\mathrm{dd}, J=7.6,5.2 \mathrm{~Hz}, 1 \mathrm{H}), 6.93(\mathrm{brs}, 1 \mathrm{H}), 6.88(\mathrm{dd}, J=5.2$, $3.6 \mathrm{~Hz}, 1 \mathrm{H}), 6.68$ (brs, 1H), 5.02 (brs, 2H), 4.03-3.89 (m, 2H), 3.07-2.92 (m, 2H), $1.40(\mathrm{~s}, 9 \mathrm{H}), 1.21(\mathrm{~d}$, $J=6.8 \mathrm{~Hz}, 3 \mathrm{H}) ;{ }^{13} \mathrm{C}$ NMR $\left(125 \mathrm{MHz}, 323 \mathrm{~K}, \mathrm{CD}_{3} \mathrm{CN}\right): \delta 175.7,156.9,156.4,148.3,146.8,135.2,128.3$, 127.8, 125.2, 125.1, 119.7, 79.9, 55.4 (br), 47.1, 43.4, 28.8, 24.9, 16.1; IR (film): $\widetilde{v}=3405$ (br), 2974, 2925, 1712, 1644, 1590, 1420, 1306, 1165, $1000 \mathrm{~cm}^{-1}$; HRMS (ESI) $m / z$ calculated for $\mathrm{C}_{20} \mathrm{H}_{25} \mathrm{O}_{3} \mathrm{~N}_{3} \mathrm{SNa}[\mathrm{M}+\mathrm{Na}]^{+}: 410.1509$, found: 410.1503; $[\alpha] \mathrm{D}^{26}$ 47.4 ( c 0.55, $\mathrm{CHCl}_{3}, 98 \%$ ee sample); HPLC analysis CHIRALPAK IF ( $\left.\phi 0.46 \mathrm{~cm} \times 25 \mathrm{~cm}\right), 2$-propanol $/ n$-hexane $=1 / 4$, flow rate $1.0 \mathrm{~mL} / \mathrm{min}$, detection at $254 \mathrm{~nm}, \mathrm{tr}_{\mathrm{R}}=23.5 \mathrm{~min}$ (major), $27.9 \mathrm{~min}$ (minor).
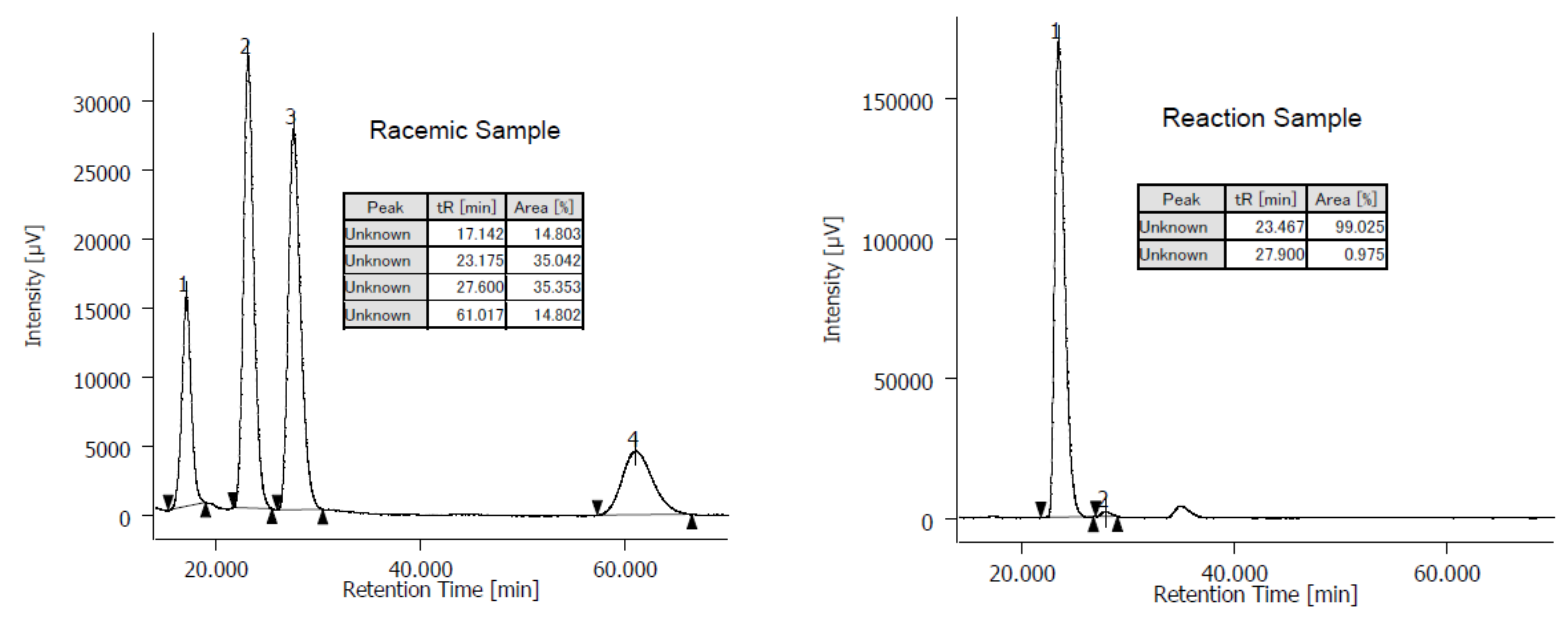

\section{tert-Butyl}

((3R,4R,E)-5-(2,3-dihydro-1H-pyrrolo[2,3-b]pyridin-1-yl)-1-(4-fluorophenyl)-4-methyl-5-oxopent-1-en-3-yl)carbamate (anti-3n)<smiles>CC(/C=C/c1ccc(F)cc1)[C@@H](NC(=O)c1ccccc1)C(=O)N1CCc2cccnc21</smiles>

(85\% yield of mixture of diastereomers (anti/syn 3.1/1), $36 \mathrm{mg}$ ). Anti-diastereomer was separated by PTLC. Amorphous solid; ${ }^{1} \mathrm{H}$ NMR $\left(500 \mathrm{MHz}, 323 \mathrm{~K}, \mathrm{CD}_{3} \mathrm{CN}\right): \delta=8.10(\mathrm{~d}, J=4.5$ $\mathrm{Hz}, 1 \mathrm{H}), 7.54(\mathrm{~d}, J=7.0 \mathrm{~Hz}, 1 \mathrm{H}), 7.29$ (brs, 2H), 7.01-6.94 (m, 3H), 6.49 (d, J = 16.0 Hz, 1H), $6.17(\mathrm{~d}, J=6.5 \mathrm{~Hz}, 1 \mathrm{H}), 6.14(\mathrm{~d}, J=6.5 \mathrm{~Hz}, 1 \mathrm{H}), 4.75$ (brs, 1H), 4.40 (brs, 1H), 4.00-3.97 (m, 2H), 3.04-2.90 (m, 2H), $1.40(\mathrm{~s}, 9 \mathrm{H}), 1.22(\mathrm{~d}, J=7.0 \mathrm{~Hz}, 3 \mathrm{H}) ;{ }^{13} \mathrm{C}$ NMR $(125 \mathrm{MHz}, 323 \mathrm{~K}$, $\left.\mathrm{CD}_{3} \mathrm{CN}\right)$ : $\delta 174.6,162.9$ (d, J = 242.9 Hz), 155.9, 155.3, 145.6, 133.9, 133.4, 130.1, 128.7, 127.9 (d, J = 7.3 Hz), 127.2, 118.4, $115.2(\mathrm{~d}, J=21.8 \mathrm{~Hz}), 78.4,56.0(\mathrm{br}), 46.0,40.8,27.6,23.8,14.6$; ${ }^{19} \mathrm{~F} \mathrm{NMR}\left(376 \mathrm{MHz}, 323 \mathrm{~K}, \mathrm{CD}_{3} \mathrm{CN}\right)$ : $\delta=-116.7$ (s, $\left.1 \mathrm{~F}\right)$; IR (film): $\widetilde{v}=3414$ (br), 2976, 2927, 1711, 1644, 1508, 1421, 1229, 1165, $967 \mathrm{~cm}^{-1}$; HRMS (ESI) $\mathrm{m} / \mathrm{z}$ calculated for $\mathrm{C}_{24} \mathrm{H}_{29} \mathrm{O}_{3} \mathrm{~N}_{3} \mathrm{FN}[\mathrm{M}+\mathrm{H}]^{+}: 426.2187$, found: 426.2181; $[\alpha]_{\mathrm{D}^{26}} 71.3$ (c $0.70, \mathrm{CHCl}_{3}, 92 \%$ ee sample); HPLC analysis CHIRALPAK IA-3 $(\phi 0.46 \mathrm{~cm} \times 25 \mathrm{~cm}), 2$-propanol $/ n$-hexane $=1 / 9$, flow rate $1.0 \mathrm{~mL} / \mathrm{min}$, detection at $254 \mathrm{~nm}, \mathrm{tR}=19.2$ $\min$ (minor), $20.8 \mathrm{~min}$ (major). 

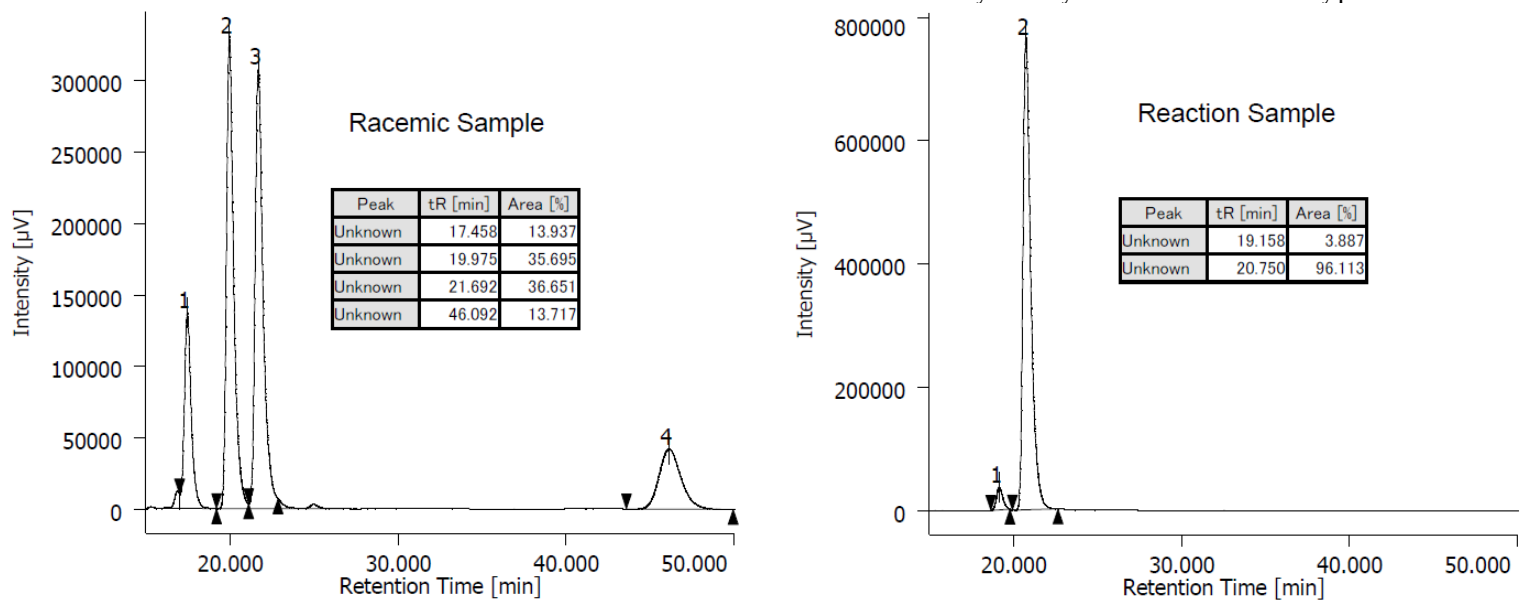

tert-Butyl ((1S,2R)-2-(2,3-dihydro-1H-pyrrolo[2,3-b]pyridine-1-carbonyl)-1-(4-fluorophenyl)butyl)carbamate (anti-3dg)<smiles>CCC(C(=O)N1CCc2cccnc21)C(NC(=O)O)c1ccc(F)cc1</smiles>

$(68 \%$ yield of mixture of diastereomers (anti/syn 8/1), $28 \mathrm{mg})$. Anti-diastereomer was separated by PTLC. Colorless oil; ${ }^{1} \mathrm{H}$ NMR (400 MHz, $\left.323 \mathrm{~K}, \mathrm{CD}_{3} \mathrm{CN}\right): \delta=8.08$ (d, J=4.8 Hz, $1 \mathrm{H}), 7.51(\mathrm{~d}, J=7.6 \mathrm{~Hz}, 1 \mathrm{H}), 7.31-7.27(\mathrm{~m}, 2 \mathrm{H}), 6.96-6.92(\mathrm{~m}, 3 \mathrm{H}), 6.67$ (brs, 1H), 4.88 (brs, 2H), 3.97-3.86 (m, 2H), 3.00-2.83 (m, 2H), 1.80-1.74 (m, 1H), 1.50 (brs, 1H), $1.35(\mathrm{~s}, 9 \mathrm{H}), 0.90(\mathrm{t}, J=$ $7.6 \mathrm{~Hz}, 3 \mathrm{H}) ;{ }^{13} \mathrm{C} \mathrm{NMR}\left(100 \mathrm{MHz}, 323 \mathrm{~K}, \mathrm{CD}_{3} \mathrm{CN}\right): \delta 175.1,162.8$ (d, J= 241.3 Hz), 156.8, 156.3, 146.4, 139.8, 135.1, 129.6 (d, J = 7.3 Hz), 128.3, 119.7, 115.7 (d, J = 21.1 Hz), 79.6, 56.9 (br), 50.0, 47.1, 28.7, 24.9, 24.8, 12.0; ${ }^{19} \mathrm{~F}$ NMR (376 MHz, $323 \mathrm{~K}, \mathrm{CD}_{3} \mathrm{CN}$ ): $\delta=-118.0$ (s, 1F); IR (film): $\widetilde{v}=3414$ (br), 2976, 2927, 1711, 1644, 1508, 1421, 1229, $1165,967 \mathrm{~cm}^{-1}$; HRMS (ESI) $\mathrm{m} / z$ calculated for $\mathrm{C}_{23} \mathrm{H}_{29} \mathrm{O}_{3} \mathrm{~N}_{3} \mathrm{~F}[\mathrm{M}+\mathrm{H}]^{+}: 414.2187$, found: 414.2180; $[\alpha]_{\mathrm{D}^{25}} 41.8\left(c 0.30, \mathrm{CHCl}_{3}\right.$, $90 \%$ ee sample); HPLC analysis CHIRALPAK IF $(\phi 0.46 \mathrm{~cm} \times 25 \mathrm{~cm}), 2$-propanol $/ n$-hexane $=1 / 6$, flow rate $0.5 \mathrm{~mL} / \mathrm{min}$, detection at $254 \mathrm{~nm}, \mathrm{t}_{\mathrm{R}}=25.5 \mathrm{~min}$ (major), $37.9 \mathrm{~min}$ (minor).
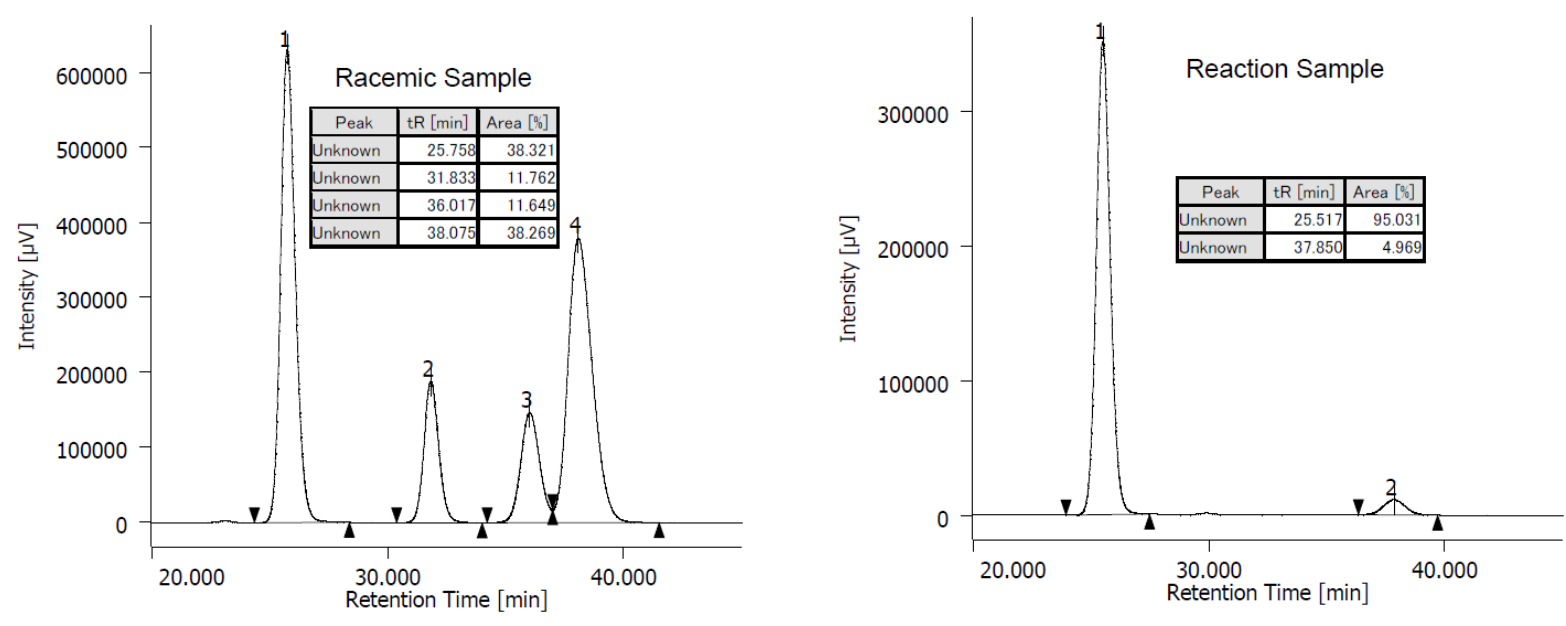

3-4. General procedure for syn-selective direct catalytic asymmetric Mannich-type reaction of propionamide 1 a and butyramide $1 d$ A flame-dried $20 \mathrm{~mL}$ test tube equipped with a magnetic stirring bar and a 3-way glass stopcock was charged with $\left(R, R_{p}\right)$-Walphos $3(\mathrm{~L} 9)(8.0 \mathrm{mg}, 0.012 \mathrm{mmol})$ and $\left[\mathrm{Cu}\left(\mathrm{CH}_{3} \mathrm{CN}\right)_{4}\right] \mathrm{PF}_{6}(3.8 \mathrm{mg}, 0.01 \mathrm{mmol})$ in a glove box. The test tube was removed from the glove box and evacuated for $10 \mathrm{~min}$. Then, it was backfilled with Ar and the corresponding amide $(0.1 \mathrm{mmol})$ and $\mathrm{N}$-Boc imine $(0.2 \mathrm{mmol})$ were added at room temperature. The test tube was cooled to $10{ }^{\circ} \mathrm{C}$ and Barton's base $(0.1 \mathrm{M}$ in THF, $0.2 \mathrm{~mL}, 0.02 \mathrm{mmol})$ was added via syringe with a stainless-steel needle. After $24 \mathrm{~h}$ of stirring, the reaction was quenched at $10{ }^{\circ} \mathrm{C}$ with a solution of $\mathrm{NH}_{4} \mathrm{Cl}(\mathrm{aq})$ followed by extraction with EtOAc ( 3 times). After evaporation of the solvent, the residue was purified by preparative TLC on silica gel with $\mathrm{CHCl}_{3} / \mathrm{EtOAc}(4: 1)$ as an eluent to give desired product. Compound syn-3dg was purified with $\mathrm{CHCl}_{3}$ : $\mathrm{EtOAc}(4 / 0.75)$. 


\section{3-5. Characterization of syn-products}

Note that the NMR spectra of the Mannich products were recorded at elevated temperature (usually $50{ }^{\circ} \mathrm{C}$ ) in $\mathrm{CD}_{3} \mathrm{CN}-d_{3}$, as rotamers were observed in $\mathrm{CDCl}_{3}$ at room temperature. ${ }^{6}$

Diasteromeric ratio (anti/syn) was determined by HPLC analysis from isolated mixture of diastereomers.

tert-Butyl ((1R,2R)-3-(2,3-dihydro-1H-pyrrolo[2,3-b]pyridin-1-yl)-2-methyl-3-oxo-1-phenylpropyl)carbamate (syn-3a)<smiles>C[C@H](NC(=O)c1ccccc1)C(=O)N1CCc2cccnc21</smiles>
( $88 \%$ yield of mixture of diastereomers (anti/syn 1/3.2), $34 \mathrm{mg}$ ). Syn-diastereomer was separated by PTLC. White solid m.p.: $165-167^{\circ} \mathrm{C}$; ${ }^{1} \mathrm{H}$ NMR $\left(400 \mathrm{MHz}, 323 \mathrm{~K}, \mathrm{CD}_{3} \mathrm{CN}\right): \delta=8.18(\mathrm{~d}, J=5.2$ $\mathrm{Hz}, 1 \mathrm{H}), 7.54-7.51(\mathrm{~m}, 1 \mathrm{H}), 7.38-7.35(\mathrm{~m}, 2 \mathrm{H}), 7.28-7.23(\mathrm{~m}, 2 \mathrm{H}), 7.21-7.17(\mathrm{~m}, 1 \mathrm{H}), 6.95(\mathrm{dd}, J=$ 7.6, $5.2 \mathrm{~Hz}, 1 \mathrm{H}), 5.72(\mathrm{br}, 1 \mathrm{H}), 5.14-5.10(\mathrm{~m}, 1 \mathrm{H}), 4.88-4.82(\mathrm{~m}, 1 \mathrm{H}), 3.98-3.90(\mathrm{~m}, 1 \mathrm{H}), 3.84-3.77$ $(\mathrm{m}, 1 \mathrm{H}), 3.00-2.84(\mathrm{~m}, 2 \mathrm{H}), 1.37(\mathrm{~s}, 9 \mathrm{H}), 1.08(\mathrm{~d}, J=6.8 \mathrm{~Hz}, 3 \mathrm{H}) ;{ }^{13} \mathrm{C}$ NMR $(100 \mathrm{MHz}, 323 \mathrm{~K}$, $\mathrm{CD}_{3} \mathrm{CN}$ ): $\delta$ 174.4, 157.0, 156.6, 146.7, 143.3, 134.9, 129.1, 128.0, 127.9, 127.8, 119.2, 79.6, 57.0 (brs), 47.0, 44.2, 28.7, 24.9, 12.7; IR (film): $\widetilde{v}=3343$ (br), 2976, 2928, 1709, 1653, 1496, 1421, 1242, $1168 \mathrm{~cm}^{-1}$; HRMS (ESI) $\mathrm{m} / \mathrm{z}$ calculated for $\mathrm{C}_{22} \mathrm{H}_{27} \mathrm{O}_{3} \mathrm{~N}_{3} \mathrm{Na}[\mathrm{M}+\mathrm{Na}]^{+}: 404.1945$, found: 404.1945; $[\alpha]_{\mathrm{D}^{25}} 1.6$ (c $1.1, \mathrm{CHCl}_{3}, 80 \%$ ee sample); HPLC analysis CHIRALPAK IF $(\phi 0.46 \mathrm{~cm} \times 25 \mathrm{~cm}), 2$-propanol $/ n$-hexane $=1 / 4$, flow rate $1.0 \mathrm{~mL} / \mathrm{min}$, detection at $254 \mathrm{~nm}, \mathrm{tR}=15.9$ $\min$ (major), $26.4 \mathrm{~min}$ (minor).
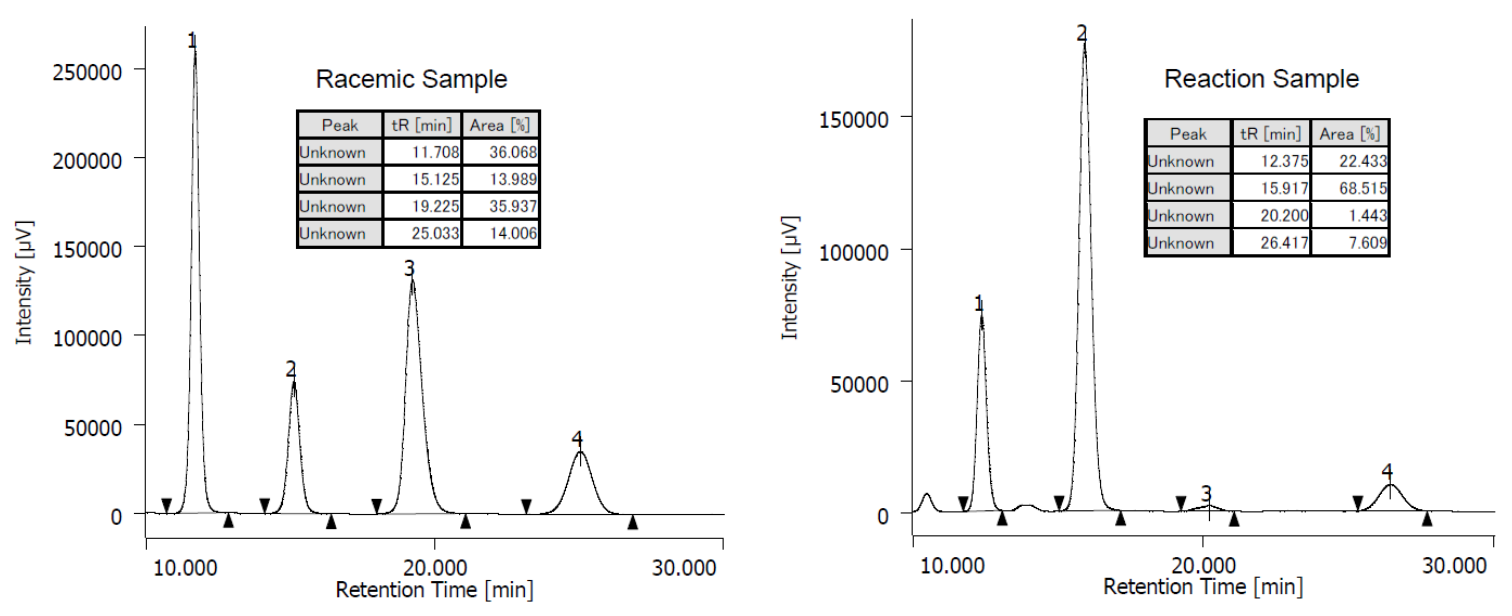

tert-Butyl ((1R,2R)-3-(2,3-dihydro-1H-pyrrolo[2,3-b]pyridin-1-yl)-1-(4-fluorophenyl)-2-methyl-3-oxopropyl) carbamate $(s y n-3 g)$<smiles>C[C@H](NC(=O)c1ccc(F)cc1)C(=O)N1CCc2cccnc21</smiles>

(85\% yield of mixture of diastereomers (anti/syn 1/2.9), $34 \mathrm{mg}$ ). Syn-diastereomer was separated by PTLC. White solid, m.p.: 224-225 ${ }^{\circ} \mathrm{C}$; ${ }^{1} \mathrm{H}$ NMR (500 MHz, $323 \mathrm{~K}$, DMSO-d6): $\delta=$ $8.15(\mathrm{~d}, J=5.0 \mathrm{~Hz}, 1 \mathrm{H}), 7.56(\mathrm{~d}, J=7.5 \mathrm{~Hz}, 1 \mathrm{H}), 7.35-7.32(\mathrm{~m}, 3 \mathrm{H}), 7.01-6.96(\mathrm{~m}, 3 \mathrm{H}), 5.01$ (brs, $1 \mathrm{H}), 4.71$ (brs, $1 \mathrm{H}), 3.88(\mathrm{ddd}, J=22.5,11.0,7.5 \mathrm{~Hz}, 1 \mathrm{H}), 3.69(\mathrm{ddd}, J=22.5,11.0,7.5 \mathrm{~Hz}, 1 \mathrm{H})$, 2.97-2.90 (m, 1H), 2.86-2.80 (m, 1H), $1.35(\mathrm{~s}, 9 \mathrm{H}), 1.06(\mathrm{~d}, J=6.0 \mathrm{~Hz}, 3 \mathrm{H}) ;{ }^{13} \mathrm{C}$ NMR $(125 \mathrm{MHz}$, 323 K, DMSO-d $)): \delta 172.6,160.7(\mathrm{~d}, J=239.3 \mathrm{~Hz}), 155.1,145.3,138.1,133.7,128.6(\mathrm{~d}, J=7.3 \mathrm{~Hz}), 126.3,118.0,114.2(\mathrm{~d}, J=$ $20.8 \mathrm{~Hz}), 77.6,55.0$ (br), 45.5, 42.9, 28.0, 23.4, 12.7; ${ }^{19} \mathrm{~F}$ NMR (376 MHz, $323 \mathrm{~K}$, DMSO-d 6 ): $\delta=-116.4$ (s, 1F); IR (film): $\tilde{v}=$ 3379 (br), 2972, 2925, 1706, 1640, 1508, 1423, 1242, $1167 \mathrm{~cm}^{-1}$; HRMS (ESI) $\mathrm{m} / z$ calculated for $\mathrm{C}_{22} \mathrm{H}_{26} \mathrm{O}_{3} \mathrm{~N}_{3} \mathrm{FNa}[\mathrm{M}+\mathrm{Na}]^{+}$: 422.1850, found: 422.1845; $[\alpha] \mathrm{D}^{26} 7.42\left(c\right.$ 0.09, $\mathrm{CHCl}_{3}, 90 \%$ ee sample); HPLC analysis CHIRALPAK IF ( $\phi 0.46 \mathrm{~cm} \times 25$ $\mathrm{cm}$ ), 2-propanol $/ n$-hexane $=1 / 4$, flow rate $1.0 \mathrm{~mL} / \mathrm{min}$, detection at $254 \mathrm{~nm}, \mathrm{tr}=14.7 \mathrm{~min}$ (major), $24.5 \mathrm{~min}$ (minor). 

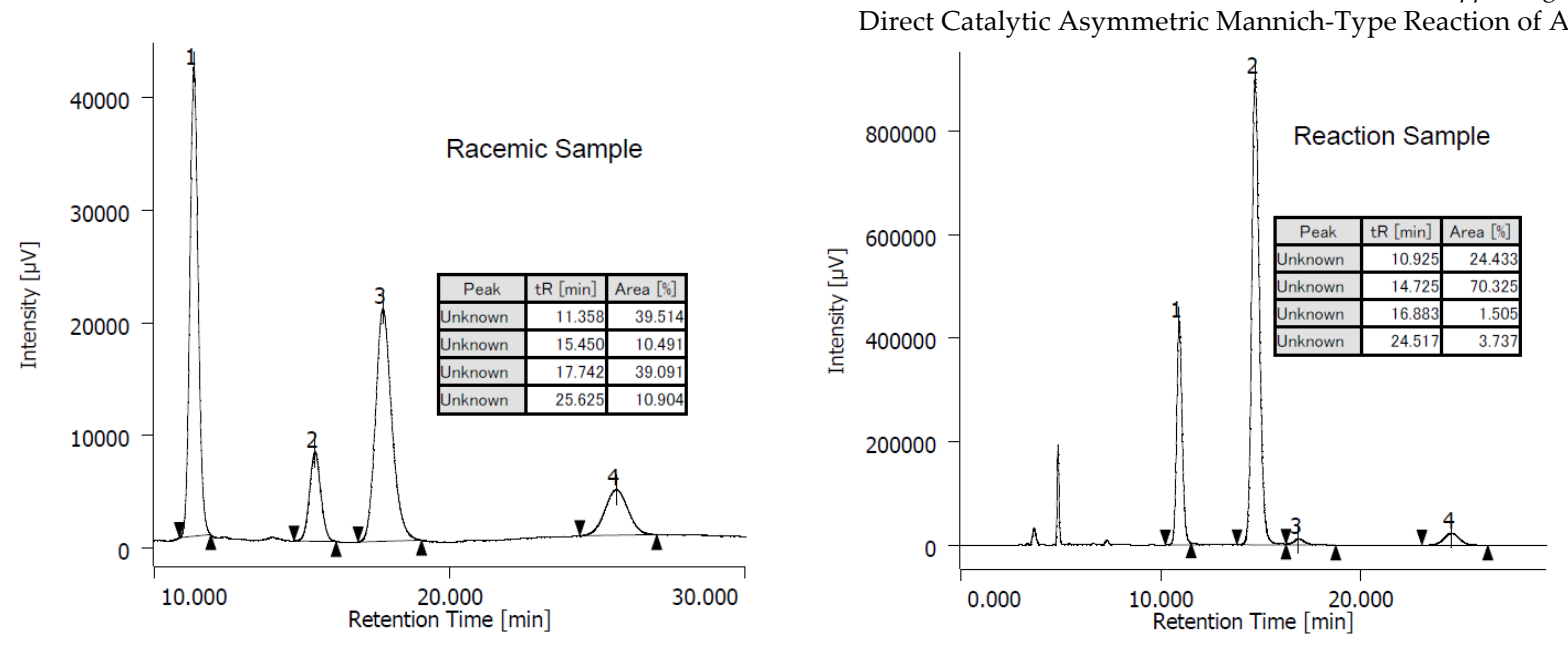

4-((1R,2R)-1-((tert-Butoxycarbonyl)amino)-3-(2,3-dihydro-1H-pyrrolo[2,3-b]pyridin-1-yl)-2-methyl-3-oxopropyl) phenyl trifluoromethanesulfonate (syn-3j)

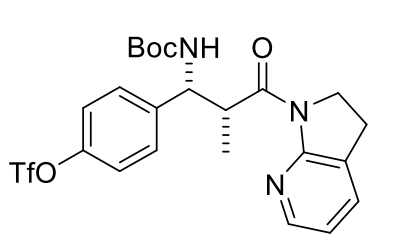

(70\% yield of mixture of diastereomers (anti/syn 1/2.2), $38 \mathrm{mg}$ ). Syn-diastereomer was separated by PTLC. Amorphous white solid; ${ }^{1} \mathrm{H}$ NMR $\left(500 \mathrm{MHz}, 323 \mathrm{~K}, \mathrm{CD}_{3} \mathrm{CN}\right): \delta=8.17$ (d, $J=5.0 \mathrm{~Hz}, 1 \mathrm{H}), 7.54-7.51(\mathrm{~m}, 3 \mathrm{H}), 7.25(J=8.5 \mathrm{~Hz}, 2 \mathrm{H}), 6.96(\mathrm{dd}, J=7.5,5.0 \mathrm{~Hz}, 1 \mathrm{H}), 5.82(\mathrm{brs}$, $1 \mathrm{H}), 5.13$ (brs, $1 \mathrm{H}), 4.89-4.84(\mathrm{~m}, 1 \mathrm{H}), 3.98-3.92(\mathrm{~m}, 1 \mathrm{H}), 3.83-3.80(\mathrm{~m}, 1 \mathrm{H}), 3.01-2.86(\mathrm{~m}, 2 \mathrm{H})$, $1.37(\mathrm{~s}, 9 \mathrm{H}), 1.09(\mathrm{~d}, J=6.5 \mathrm{~Hz}, 3 \mathrm{H}) ;{ }^{13} \mathrm{C}$ NMR $\left(125 \mathrm{MHz}, 323 \mathrm{~K}, \mathrm{CD}{ }_{3} \mathrm{CN}\right): \delta 174.0,156.9,156.6$, 149.5, 146.7, 144.3, 135.0, 130.0, 128.0, 122.0, 119.9 (q, $J=318.6 \mathrm{~Hz}), 119.4,80.0,56.7$ (br), 47.0, 44.0, 29.0, 24.8, 12.8; ${ }^{19} \mathrm{~F}$ NMR (376 MHz, $323 \mathrm{~K}, \mathrm{CD}_{3} \mathrm{CN}$ ): $\delta=-74.0$ (s, 3F); IR (film): $\widetilde{v}=3339$ (br), 2976, 2925, 2853, 1706, 1650, 1591, 1500, 1421, 1213, 1141, $889 \mathrm{~cm}^{-1}$; HRMS (ESI) $\mathrm{m} / z$ calculated for $\mathrm{C}_{23} \mathrm{H}_{27} \mathrm{O}_{6} \mathrm{~N}_{3} \mathrm{~F}_{3} \mathrm{~S}[\mathrm{M}+\mathrm{H}]^{+}: 530.1567$, found: 530.1561; [ $\left.\alpha\right]_{\mathrm{D}^{26}} 2.7$ (c 0.35, $\mathrm{CHCl}_{3}, 83 \%$ ee sample); HPLC analysis CHIRALPAK IF $(\phi 0.46 \mathrm{~cm} \times 25 \mathrm{~cm}), 2$-propanol $/ n$-hexane = 1/4, flow rate 1.0 $\mathrm{mL} / \mathrm{min}$, detection at $254 \mathrm{~nm}, \mathrm{t}_{\mathrm{R}}=15.0 \mathrm{~min}$ (major), $24.5 \mathrm{~min}$ (minor).
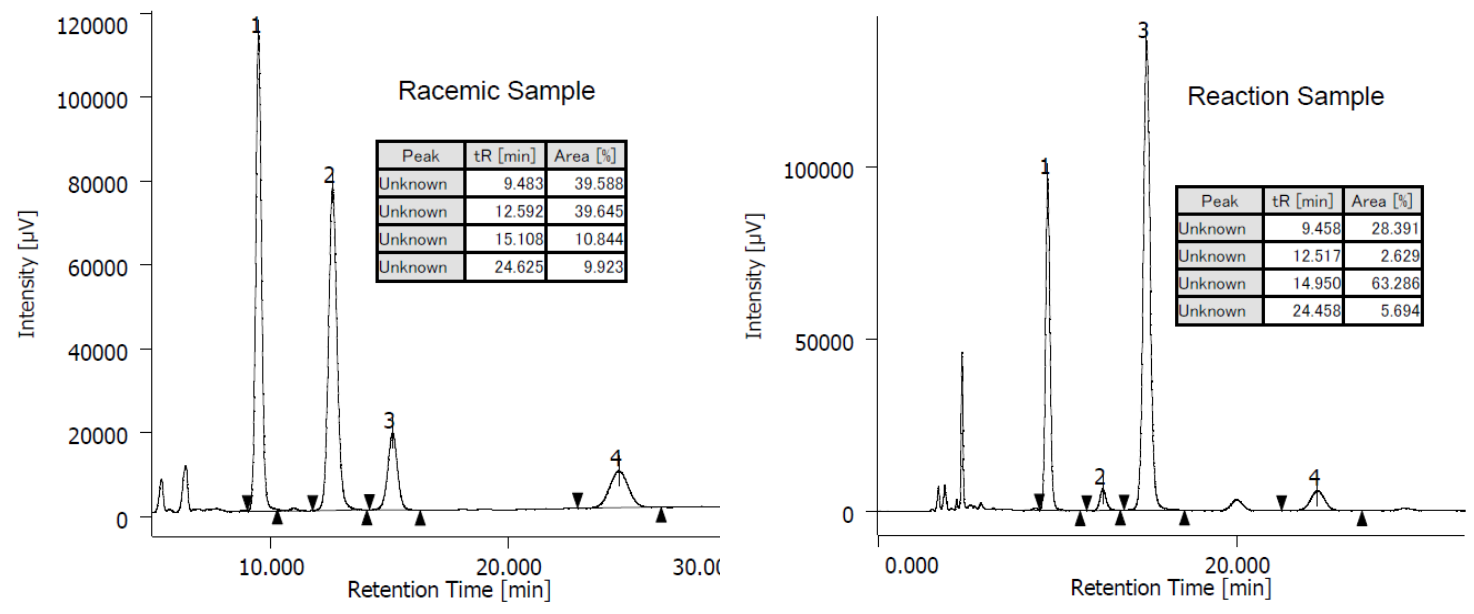

tert-Butyl ((1R,2R)-3-(2,3-dihydro-1H-pyrrolo[2,3-b]pyridin-1-yl)-2-methyl-3-oxo-1-(4-(trifluoromethyl)phenyl) propyl)carbamate (syn-3o)<smiles>CC(C(=O)N1CCc2cccnc21)[C@H](NC(=O)c1ccccc1)c1ccc(C(F)(F)F)cc1</smiles>

(79\% yield of mixture of diastereomers (anti/syn 1/3.4), $40 \mathrm{mg}$ ). Syn-diastereomer was separated by PTLC. White solid, m.p.: $154-156{ }^{\circ} \mathrm{C} ;{ }^{1} \mathrm{H}$ NMR $\left(500 \mathrm{MHz}, 323 \mathrm{~K}, \mathrm{CD}_{3} \mathrm{CN}\right): \delta=$ $8.18(\mathrm{~d}, J=5.0 \mathrm{~Hz}, 1 \mathrm{H}), 7.58-7.52(\mathrm{~m}, 5 \mathrm{H}), 6.96(\mathrm{dd}, J=7.5,5.0 \mathrm{~Hz}, 1 \mathrm{H}), 5.86$ (brs, $1 \mathrm{H}), 5.18$ (brs, 1H), 4.90-4.85 (m, 1H), $3.95(\mathrm{ddd}, J=22.5,12.0,7.0 \mathrm{~Hz}, 1 \mathrm{H}), 3.82(\mathrm{ddd}, J=22.5,12.0,6.0$ $\mathrm{Hz}, 1 \mathrm{H}), 3.01-2.84(\mathrm{~m}, 2 \mathrm{H}), 1.37$ (s, 9H), 1.09 (d, J = 7.0 Hz, 3H); ${ }^{13} \mathrm{C}$ NMR (125 MHz, $323 \mathrm{~K}$, $\left.\mathrm{CD}_{3} \mathrm{CN}\right): \delta 173.9,156.9,156.6,147.9,146.7,135.0,129.5(\mathrm{q}, J=31.6 \mathrm{~Hz}), 128.0,125.6(\mathrm{q}, J=269.0 \mathrm{~Hz}), 126.0,119.4,79.9$, 56.9 (br), 47.0, 44.0, 28.7, 24.8, 12.7; ${ }^{19} \mathrm{~F}$ NMR (376 MHz, $\left.323 \mathrm{~K}, \mathrm{CD}_{3} \mathrm{CN}\right): \delta=-63.0$ (s, 1F); IR (film): $\widetilde{v}=3340$ (br), 2977, 2925, 2853, 1620, 1651, 1591, 1423, 1327,1165, 1123, $1068 \mathrm{~cm}^{-1}$; HRMS (ESI) $\mathrm{m} / z$ calculated for $\mathrm{C}_{23} \mathrm{H}_{27} \mathrm{O}_{3} \mathrm{~N}_{3} \mathrm{~F}_{3}[\mathrm{M}+\mathrm{H}]^{+}$: 
450.1999, found: 450.1995; $[\alpha]_{\mathrm{D}^{26}}-1.9$ (c 0.45, $\mathrm{CHCl}_{3}, 83 \%$ ee sample); HPLC analysis CHIRALPAK IC ( $\phi 0.46 \mathrm{~cm} \times 25$ $\mathrm{cm}$ ), 2-propanol $/ n$-hexane $=1 / 13$, flow rate $1.0 \mathrm{~mL} / \mathrm{min}$, detection at $254 \mathrm{~nm}, \mathrm{t}=17.9$ min (minor), $36.9 \mathrm{~min}$ (major).
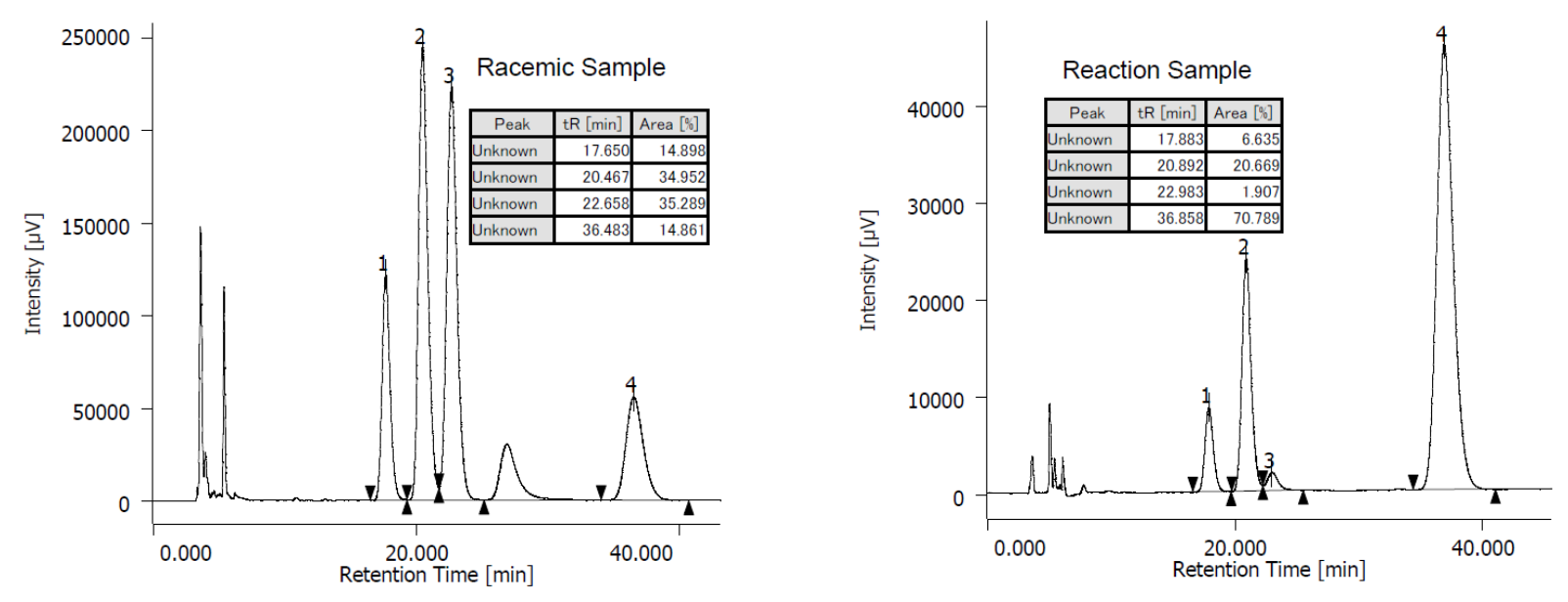

tert-Butyl ((1R,2R)-2-(2,3-dihydro-1H-pyrrolo[2,3-b]pyridine-1-carbonyl)-1-(4-fluorophenyl)butyl)carbamate (syn-3dg)<smiles>CCC(C(=O)N1CCc2cccnc21)[C@H](NC(=O)O)c1ccc(F)cc1</smiles>

(72\% yield of mixture of diastereomers (anti/syn 1/3.0), $30 \mathrm{mg}$ ). Syn-diastereomer was separated by PTLC. White solid, m.p.: $182-184{ }^{\circ} \mathrm{C} ;{ }^{1} \mathrm{H}$ NMR $\left(500 \mathrm{MHz}, 323 \mathrm{~K}, \mathrm{CD}_{3} \mathrm{CN}\right): \delta=$ $8.15(\mathrm{~d}, J=5.5 \mathrm{~Hz}, 1 \mathrm{H}), 7.52(\mathrm{~d}, J=7.5 \mathrm{~Hz}, 1 \mathrm{H}), 7.35-7.32(\mathrm{~m}, 2 \mathrm{H}), 6.96-6.91(\mathrm{~m}, 3 \mathrm{H}), 5.77$ (brs, $1 \mathrm{H}), 4.93(\mathrm{brs}, 2 \mathrm{H}), 3.94(\mathrm{ddd}, J=22.5,12.0,6.5 \mathrm{~Hz}, 1 \mathrm{H}), 3.77(\mathrm{ddd}, J=22.5,10.5,6.0 \mathrm{~Hz}, 1 \mathrm{H})$, 2.97-2.91 (m, 1H), 2.87-2.80 (m, 1H), 1.87-1.78 (m, 1H), $1.53(\mathrm{brs}, 1 \mathrm{H}), 1.37(\mathrm{~s}, 9 \mathrm{H}), 0.83(\mathrm{t}, J=$ $7.5 \mathrm{~Hz}, 3 \mathrm{H}) ;{ }^{13} \mathrm{C}$ NMR $\left(125 \mathrm{MHz}, 323 \mathrm{~K}, \mathrm{CD}_{3} \mathrm{CN}\right): \delta$ 173.5, 162.8 (d, J = 241.1 Hz), 156.9, 156.5, 146.5, 139.5, 134.9, 129.8 (d, $J=8.1 \mathrm{~Hz}), 128.0,119.4,115.5(\mathrm{~d}, J=21.6 \mathrm{~Hz}), 79.7,56.6(\mathrm{br}), 51.0,47.0,28.7,24.8,22.0,12.1 ;{ }^{19} \mathrm{~F} \mathrm{NMR}(376 \mathrm{MHz}, 323 \mathrm{~K}$, $\mathrm{CD}_{3} \mathrm{CN}$ ): $\delta=-118.0$ (s, 1F); IR (film): $\widetilde{v}=3340$ (br), 2964, 2924, 2852, 1706, 1644, 1602, 1590, 1509, 1421, 1308, 1165,1013 $\mathrm{cm}^{-1}$; HRMS (ESI) $m / z$ calculated for $\mathrm{C}_{23} \mathrm{H}_{29} \mathrm{O}_{3} \mathrm{~N}_{3} \mathrm{~F}[\mathrm{M}+\mathrm{H}]^{+}: 414.2187$, found: 414.2180; $[\alpha]^{26} 15.5$ (c 0.30, $\mathrm{CHCl}_{3}, 95 \%$ ee sample); HPLC analysis CHIRALPAK IF $(\phi 0.46 \mathrm{~cm} \times 25 \mathrm{~cm}), 2$-propanol $/ n$-hexane $=1 / 6$, flow rate $0.5 \mathrm{~mL} / \mathrm{min}$, detection at $254 \mathrm{~nm}, \mathrm{t}_{\mathrm{R}}=31.8 \mathrm{~min}$ (major), $36.0 \mathrm{~min}$ (minor).
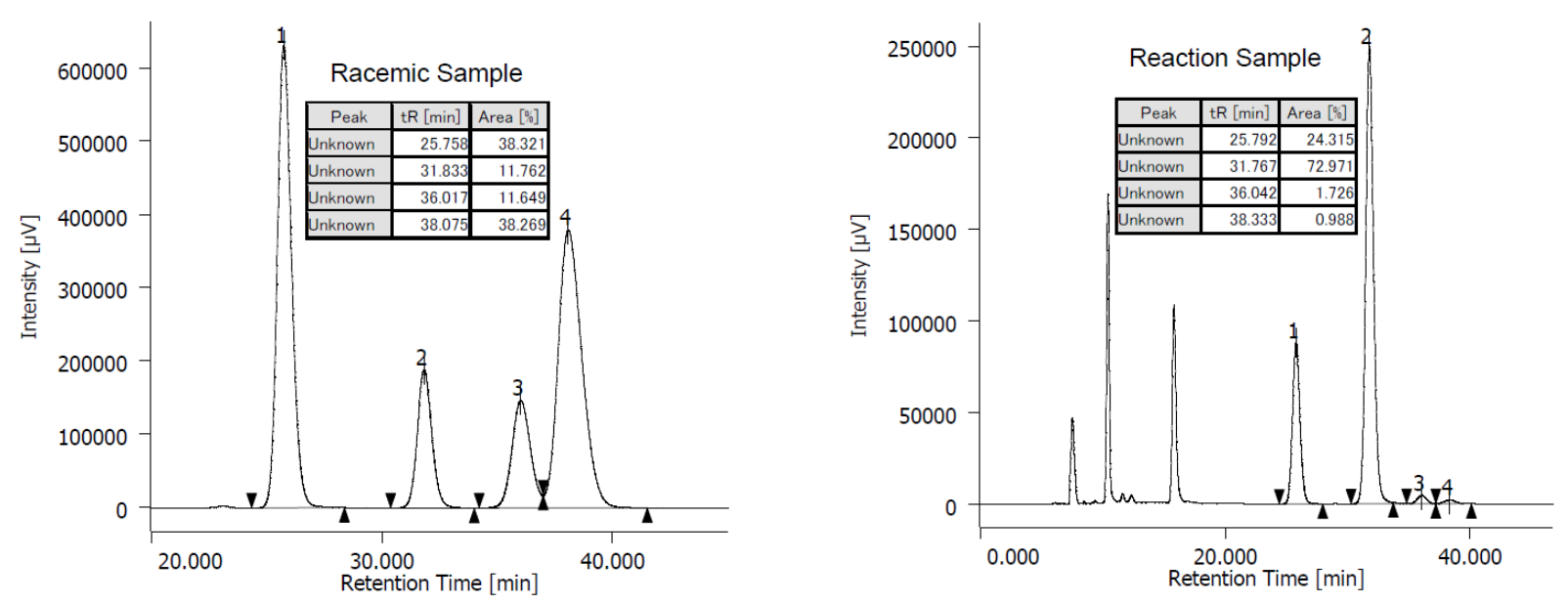

3-6. General procedure for direct catalytic asymmetric Mannich-type reaction of acetamide 1e

A flame-dried $20 \mathrm{~mL}$ test tube equipped with a magnetic stirring bar and a 3-way glass stopcock were charged with $\left(R, R_{p}\right)$-Walphos 3 (L9) $(16.1 \mathrm{mg}, 0.024 \mathrm{mmol})$ and $\left[\mathrm{Cu}\left(\mathrm{CH}_{3} \mathrm{CN}\right)_{4}\right] \mathrm{PF}_{6}(7.45 \mathrm{mg}, 0.02 \mathrm{mmol})$ in a glove box. The test tube was removed from the glove box and evacuated for $10 \mathrm{~min}$. Then, it was backfilled with Ar and DME (0.15 mL) was added via syringe with a stainless-steel needle at room temperature. The resulting clear brown solution was stirred for $1 \mathrm{~h}$. The mixture was transferred to another test tube prepared as follows: A flame dried $20 \mathrm{~mL}$ test tube equipped with 

a magnetic stirring bar and a 3-way glass stopcock were charged with 7-azaindoline acetamide 1e (0.3 mmol) and $\mathrm{N}$-Boc imine $(0.2 \mathrm{mmol})$. The test tube was stirred for $2 \mathrm{~min}$ at $40{ }^{\circ} \mathrm{C}$. To the mixture was added Barton's base $(0.2 \mathrm{M}$ in DME, $0.1 \mathrm{~mL}, 0.02 \mathrm{mmol}$ ) via syringe with a stainless-steel needle. After $18 \mathrm{~h}$ of stirring at $40{ }^{\circ} \mathrm{C}$, the reaction was quenched with solution of acetic acid in DME (0.1 M in DME, $1 \mathrm{~mL})$. After evaporation of the solvent under reduced pressure, the residue was purified by preparative TLC on silica gel with $\mathrm{CH}_{2} \mathrm{Cl}_{2} / \mathrm{Et}_{2} \mathrm{O} / n$-hexane/EtOAc (8:4:4:1) as an eluent to give desired product 4 .

\section{3-7. Characterization of acetamide products}

Note that the NMR spectra of the Mannich products were recorded at elevated temperature (usually $\left.50{ }^{\circ} \mathrm{C}\right)$ in $\mathrm{CD}_{3} \mathrm{CN}-\mathrm{d}_{3}$, as rotamers were observed in $\mathrm{CDCl}_{3}$ at room temperature. ${ }^{6}$

\section{tert-Butyl (R)-(3-(2,3-dihydro-1H-pyrrolo[2,3-b]pyridin-1-yl)-3-oxo-1-phenylpropyl)carbamate (4a)}

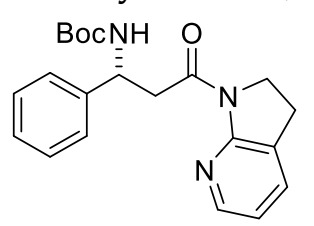

(53\% yield, $39 \mathrm{mg})$. White solid, m.p.: $135-137{ }^{\circ} \mathrm{C}$; ${ }^{1} \mathrm{H}$ NMR $\left(400 \mathrm{MHz}, 323 \mathrm{~K}, \mathrm{CD}_{3} \mathrm{CN}\right): \delta=8.11(\mathrm{~d}$, $J=4.4 \mathrm{~Hz}, 1 \mathrm{H}), 7.55(\mathrm{~d}, J=6.8 \mathrm{~Hz}, 1 \mathrm{H}), 7.41-7.22(\mathrm{~m}, 5 \mathrm{H}), 6.96-6.93(\mathrm{~m}, 1 \mathrm{H}), 6.11(\mathrm{brs}, 1 \mathrm{H}), 5.15$ (brs, $1 \mathrm{H}), 3.99(\mathrm{t}, J=8.4 \mathrm{~Hz}, 2 \mathrm{H}), 3.63(\mathrm{dd}, J=14.4,9.2 \mathrm{~Hz}, 1 \mathrm{H}), 3.47(\mathrm{dd}, J=14.4,4.4 \mathrm{~Hz}, 1 \mathrm{H}), 3.02$ $(\mathrm{t}, J=8.4 \mathrm{~Hz}, 2 \mathrm{H}), 1.35$ (s, 9H); ${ }^{13} \mathrm{C} \mathrm{NMR}\left(100 \mathrm{MHz}, 323 \mathrm{~K}, \mathrm{CD}_{3} \mathrm{CN}\right): \delta$ 170.5, 157.1, 156.3, 146.7, 144.7, 135.0, 129.4, 128.0, 127.9, 127.3, 119.3, 79.6, 53.2 (br), 46.9, 43.8, 28.7, 25.0; IR (film): $\widetilde{v}=3020$, 2360, 1711, 1419, 1419, $1362 \mathrm{~cm}^{-1}$; HRMS (ESI) $m / z$ calculated for $\mathrm{C}_{21} \mathrm{H}_{26} \mathrm{O}_{3} \mathrm{~N}_{3}[\mathrm{M}+\mathrm{H}]^{+}: 368.1969$, found: 368.1963; $[\alpha] \mathrm{D}^{25}-$ 24.8 ( c 0.1, $\mathrm{CHCl}_{3}, 88 \%$ ee sample); HPLC analysis CHIRALPAK IA-3 $(\phi 0.46 \mathrm{~cm} \mathrm{x} 25 \mathrm{~cm}), 2-$ propanol $/ n$-hexane $=1 / 9$, flow rate $1.0 \mathrm{~mL} / \mathrm{min}$, detection at $254 \mathrm{~nm}, \mathrm{t}_{\mathrm{R}}=23.8 \mathrm{~min}$ (minor), $26.1 \mathrm{~min}$ (major).
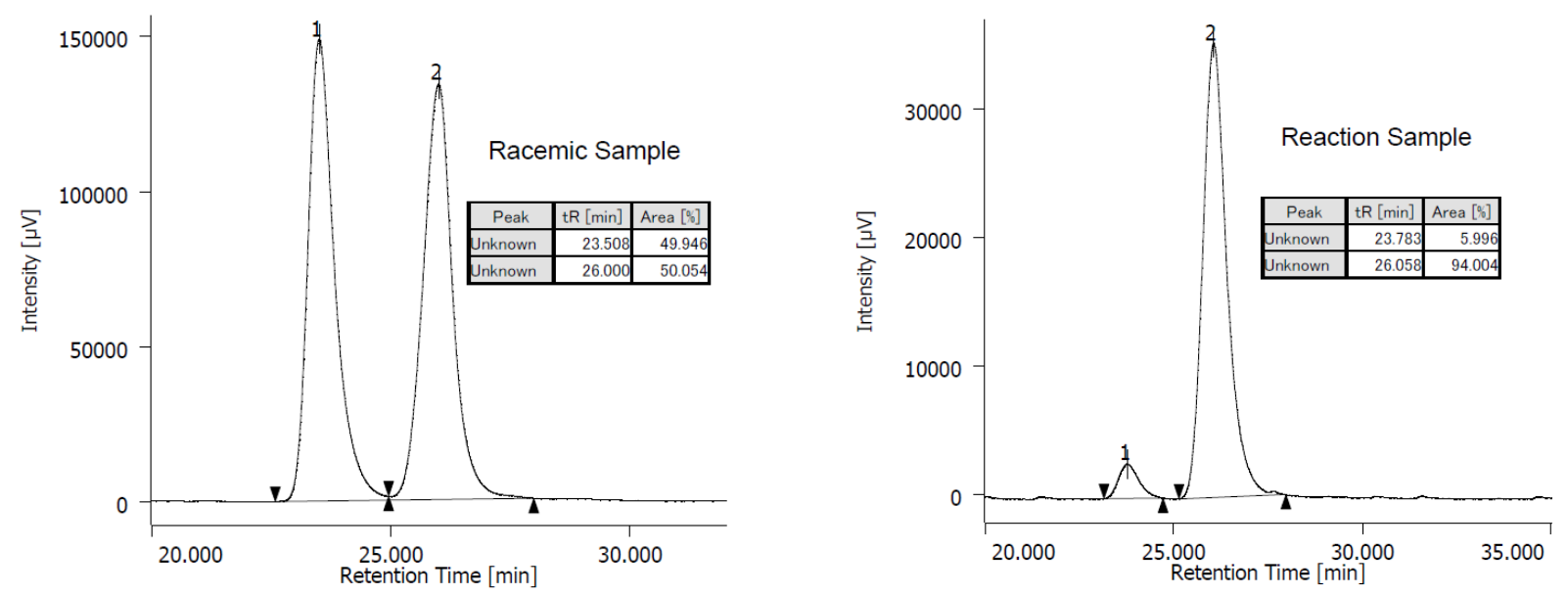

\section{tert-Butyl (R)-(3-(2,3-dihydro-1H-pyrrolo[2,3-b]pyridin-1-yl)-3-oxo-1-(p-tolyl)propyl)carbamate (4b)}

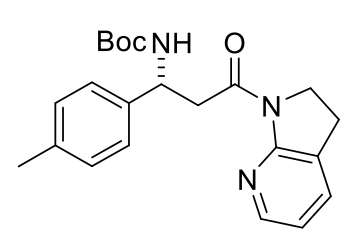

(55\% yield, $42 \mathrm{mg}$ ). White solid, m.p.: $136-138{ }^{\circ} \mathrm{C}$; ${ }^{1} \mathrm{H}$ NMR $(400 \mathrm{MHz}, 323 \mathrm{~K}, \mathrm{CD} 3 \mathrm{CN}): \delta=$ 8.10 (brs, 1H), 7.55 (d, J = 7.2 Hz, 1H), 7.27 (d, J = 8.0 Hz, 2H), 7.14 (d, J = 7.6 Hz, 2H), 6.94 (brs, $1 \mathrm{H}), 6.06$ (brs, 1H), 5.09 (brs, 1H), 3.98 (t, J = 8.0 Hz, 2H), 3.60 (brs, 1H), 3.46 (brs, 1H), 3.01 (t, J $=8.4 \mathrm{~Hz}, 2 \mathrm{H}), 2.30(\mathrm{~s}, 3 \mathrm{H}), 1.35(\mathrm{~s}, 9 \mathrm{H}) ;{ }^{13} \mathrm{C} \mathrm{NMR}\left(100 \mathrm{MHz}, 323 \mathrm{~K}, \mathrm{CD}_{3} \mathrm{CN}\right): \delta$ 170.6, 157.1, 156.3, 146.7, 141.7, 137.7, 134.9, 130.0, 128.0, 127.3, 119.3, 79.6, 53.0 (br), 46.9, 43.8, 28.7, 25.0, 21.2; IR (film): $\tilde{v}=3019,2418,1749,1711,1421,1363,1222,1091 \mathrm{~cm}^{-1}$; HRMS (ESI) m/z calculated for $\mathrm{C}_{22} \mathrm{H}_{28} \mathrm{O}_{3} \mathrm{~N}_{3}$ $[\mathrm{M}+\mathrm{H}]^{+}: 382.2125$, found: $382.2116 ;[\alpha]_{\mathrm{D}^{25}}-24.6\left(c\right.$ 0.1, $\mathrm{CHCl}_{3}, 81 \%$ ee sample); HPLC analysis CHIRALPAK IA-3 $(\phi 0.46$ $\mathrm{cm} \times 25 \mathrm{~cm}$ ), 2-propanol $/ n$-hexane $=1 / 9$, flow rate $1.0 \mathrm{~mL} / \mathrm{min}$, detection at $254 \mathrm{~nm}, \mathrm{t}=24.9 \mathrm{~min}(\mathrm{major}), 27.7 \mathrm{~min}$ (minor). 


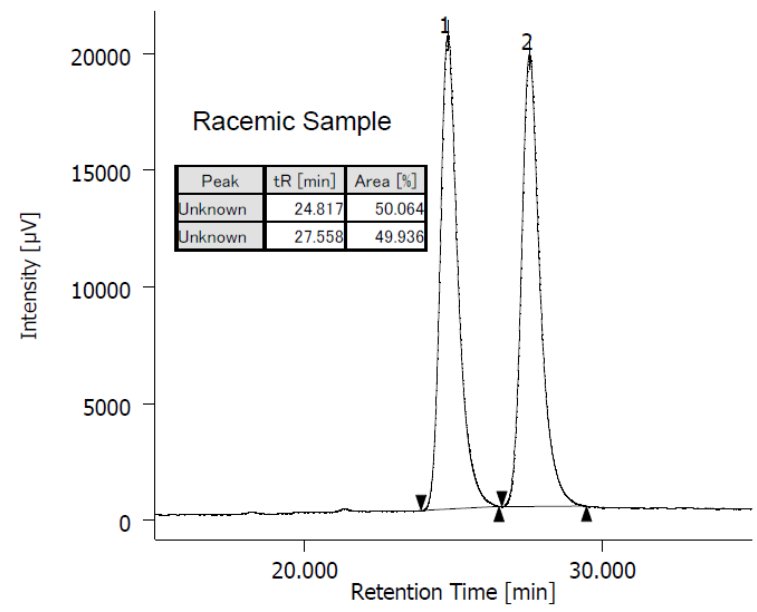

Direct Catalytic Asymmetric Mannich-Type Reaction of Alkylamides

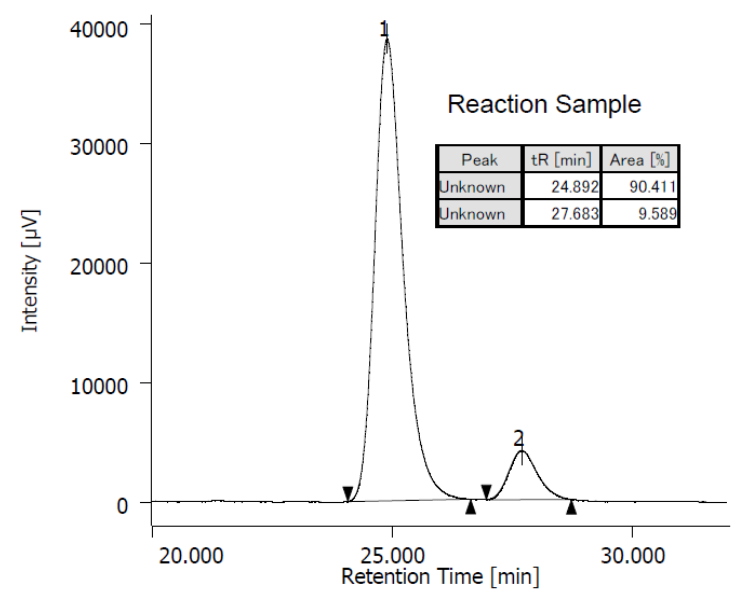

tert-Butyl (R)-(3-(2,3-dihydro-1H-pyrrolo[2,3-b]pyridin-1-yl)-1-(4-fluorophenyl)-3-oxopropyl)carbamate (4g)<smiles>O=C(N[C@@H](CC(=O)N1CCc2cccnc21)c1ccc(F)cc1)c1ccccc1</smiles>

(50\% yield, $38.5 \mathrm{mg})$. White solid, m.p.: $151-153{ }^{\circ} \mathrm{C} ;{ }^{1} \mathrm{H}$ NMR $(400 \mathrm{MHz}, 323 \mathrm{~K}, \mathrm{CD} 3 \mathrm{CN}): \delta=$ $8.10(\mathrm{~d}, J=4.0 \mathrm{~Hz}, 1 \mathrm{H}), 7.55(\mathrm{~d}, J=7.2 \mathrm{~Hz}, 1 \mathrm{H}), 7.41(\mathrm{dd}, J=8.4,5.6 \mathrm{~Hz}, 2 \mathrm{H}), 7.07-7.03(\mathrm{~m}, 2 \mathrm{H})$, 6.95 (brs, 1H), 6.11 (brs, 1H), 5.13 (brs, 1H), 3.98 (t, J = 8.4 Hz, 2H), 3.61 (dd, J = 14.8, 8.8 Hz, $1 \mathrm{H}), 3.47(\mathrm{dd}, J=14.8,8.8 \mathrm{~Hz}, 1 \mathrm{H}), 3.02(\mathrm{t}, J=8.4 \mathrm{~Hz}, 2 \mathrm{H}), 1.35(\mathrm{~s}, 9 \mathrm{H}) ;{ }^{13} \mathrm{C} \mathrm{NMR}(100 \mathrm{MHz}$, $\left.323 \mathrm{~K}, \mathrm{CD}{ }_{3} \mathrm{CN}\right): \delta 170.4,162.9(\mathrm{~d}, J=241.3 \mathrm{~Hz}), 157.0,156.2,146.7,140.8,135.0,129.2(\mathrm{~d}, J=8.1$ $\mathrm{Hz}), 128.0,119.4,115.9(\mathrm{~d}, J=21.9 \mathrm{~Hz}), 79.7,52.7(\mathrm{br}), 46.8,43.7,28.7,25.0 ;{ }^{19} \mathrm{~F} \mathrm{NMR}\left(376 \mathrm{MHz}, 323 \mathrm{~K}, \mathrm{CD}{ }_{3} \mathrm{CN}\right): \delta=-$ 117.9 (s, 1F); IR (film): $\widetilde{v}=3019,1749,1710,1421,1363,1091 \mathrm{~cm}^{-1}$; HRMS (ESI) $\mathrm{m} / z$ calculated for $\mathrm{C}_{21} \mathrm{H}_{25} \mathrm{O}_{3} \mathrm{~N}_{3} \mathrm{~F}[\mathrm{M}+\mathrm{H}]^{+}$: 386.1874, found: 386.1869; $[\alpha]_{\mathrm{D}^{25}}-36.3$ ( $0.07, \mathrm{CHCl}_{3}, 92 \%$ ee sample); HPLC analysis CHIRALPAK IA-3 ( $\phi 0.46 \mathrm{~cm} \times 25$ $\mathrm{cm}$ ), 2-propanol $/ n$-hexane $=1 / 9$, flow rate $1.0 \mathrm{~mL} / \mathrm{min}$, detection at $254 \mathrm{~nm}, \mathrm{t}_{\mathrm{R}}=23.9 \mathrm{~min}$ (major), $26.1 \mathrm{~min}(\mathrm{minor})$.
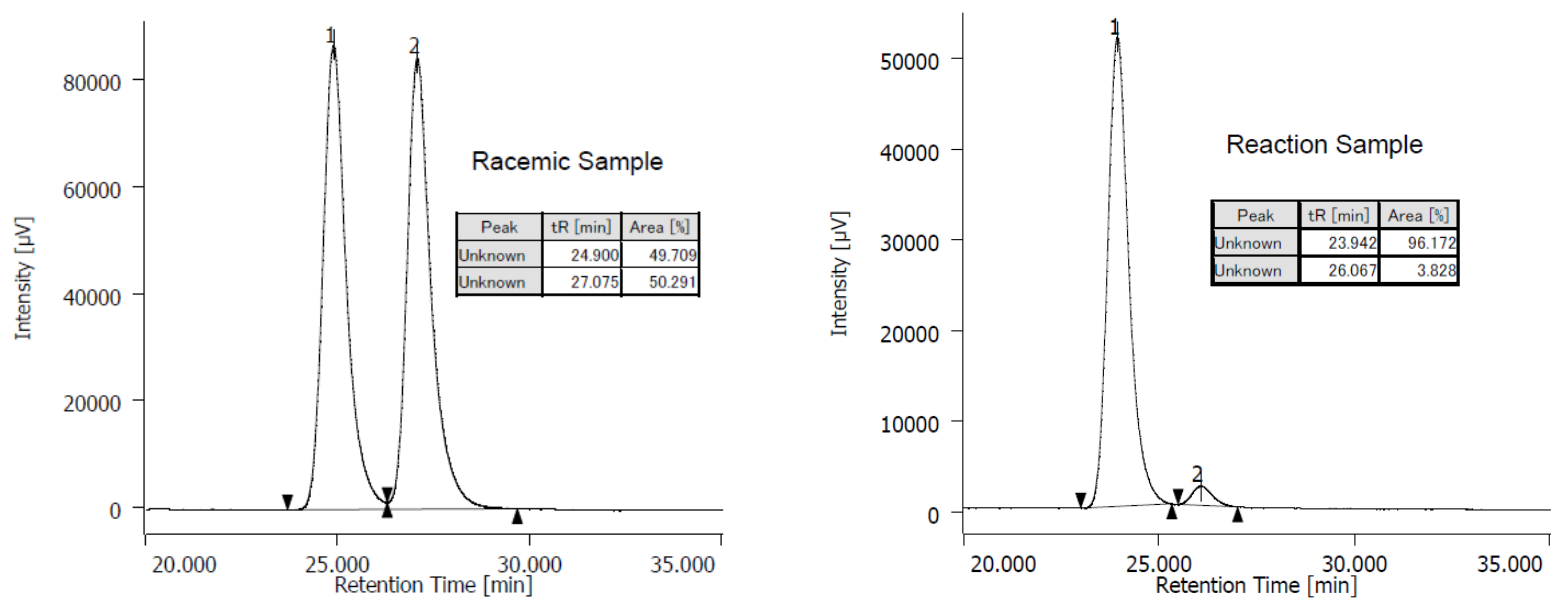

\section{tert-Butyl (R)-(1-(4-chlorophenyl)-3-(2,3-dihydro-1H-pyrrolo[2,3-b]pyridin-1-yl)-3-oxopropyl)carbamate (4p)}<smiles>O=C(N[C@@H](CC(=O)N1CCc2cccnc21)c1ccc(Cl)cc1)c1ccccc1</smiles>
(42\% yield, $37 \mathrm{mg})$. White solid, m.p.: $184-186{ }^{\circ} \mathrm{C}$; ${ }^{1} \mathrm{H}$ NMR $(500 \mathrm{MHz}, 323 \mathrm{~K}, \mathrm{CD} 3 \mathrm{CN}): \delta=$ 8.10 (brs, 1H), 7.56 (d, J = 8.0 Hz, 1H), 7.38 (brs, 2H), 7.32 (brs, 2H), 6.95 (brs, 1H), 6.11 (brs, 1H), 5.11 (brs, 1H), 3.99 (t, $J=8.5 \mathrm{~Hz}, 2 \mathrm{H}), 3.61$ (dd, $J=14.5,8.5 \mathrm{~Hz}, 1 \mathrm{H}), 3.47$ (brs, $1 \mathrm{H}), 3.02(\mathrm{t}$, $J=8.5 \mathrm{~Hz}, 2 \mathrm{H}), 1.35$ (s, 9H); ${ }^{13} \mathrm{C}$ NMR (125 MHz, $\left.323 \mathrm{~K}, \mathrm{CD}_{3} \mathrm{CN}\right): \delta$ 170.4, 157.1, 156.2, 146.7,

143.6, 135.0, 133.2, 129.4, 129.1, 128.0, 119.4, 79.8, 52.7 (br), 46.8, 43.5, 28.7, 25.0; IR (film): $\widetilde{v}=$ 3019, 2400, 1523, 1423, 1211, $1046 \mathrm{~cm}^{-1}$; HRMS (ESI) $\mathrm{m} / z$ calculated for $\mathrm{C}_{21} \mathrm{H}_{25} \mathrm{O}_{3} \mathrm{~N}_{3} \mathrm{Cl}[\mathrm{M}+\mathrm{H}]^{+}$: 402.1579, found: 402.1569; $[\alpha]_{\mathrm{D}}^{26}-32.6$ ( c 0.4, $\mathrm{CHCl}_{3}, 88 \%$ ee sample); HPLC analysis CHIRALPAK AD-3 ( $\left.\phi 0.46 \mathrm{~cm} \times 25 \mathrm{~cm}\right), 2$-propanol/n-hexane $=1 / 9$, flow rate $1.0 \mathrm{~mL} / \mathrm{min}$, detection at $254 \mathrm{~nm}, \mathrm{tr}=34.7 \mathrm{~min}$ (major), $49.0 \mathrm{~min}$ (minor). 

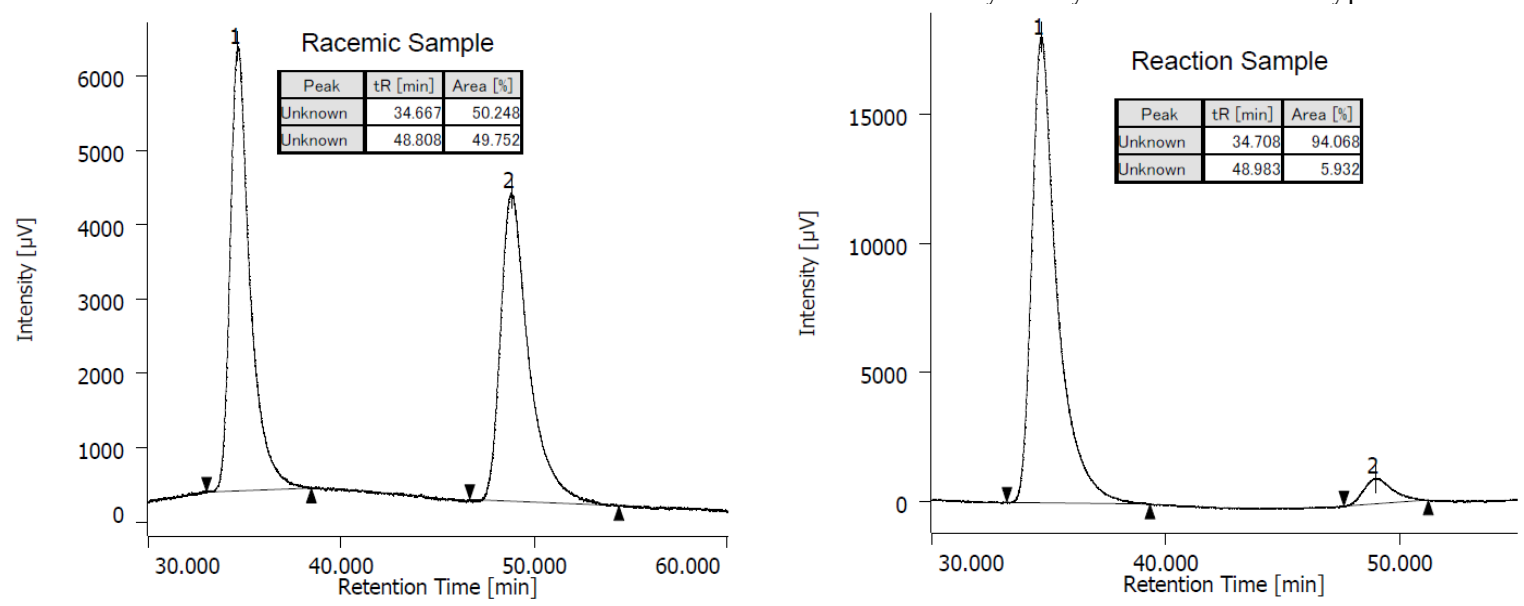


\section{Determination of absolute configuration}

\section{4-1. anti-Mannich adduct}

Single crystals of anti-3g were obtained by slow evaporation from solution in EtOAc at rt. A suitable crystal was selected and the sample was measured on a Rigaku R-AXIS RAPID diffractometer using graphite monochromated $\mathrm{Cu}-\mathrm{K} \alpha$ radiation. The data were collected at $93 \mathrm{~K}$. Refined structure and crystallographic parameters are summarized in Figure S6 and Table S5. CCDC 1469658 contains the supplementary crystallographic data for anti-3g. Absolute configuration of anti-3g was determined as $2 R, 3 S$ and the absolute configuration of other products $\mathbf{3 a - f}, \mathbf{3 h}-\mathbf{n}$, and $\mathbf{3 d g}$ were deduced by analogy.

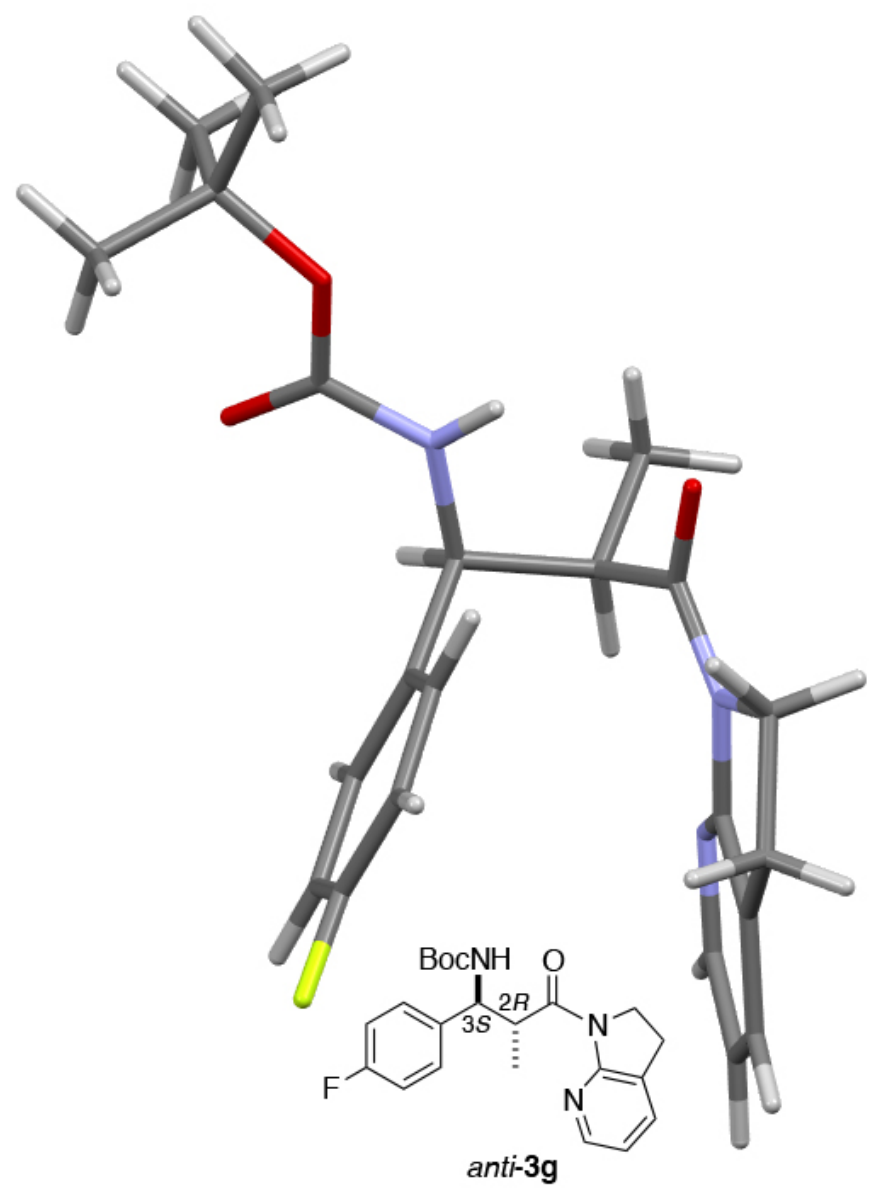

Figure S6. Structure of anti-3g. Color code; white: hydrogen, gray: carbon, blue: nitrogen, red: oxygen; light green: fluorine.
Table S5. Selected crystal data of anti-3g. Empirical Formula $\quad \mathrm{C}_{22} \mathrm{H}_{26} \mathrm{O}_{3} \mathrm{~N}_{3} \mathrm{~F}$ Formula Weight 399.46 Crystal Color, Habit colorless, block Crystal Dimensions Crystal System Lattice Parameters

$\begin{array}{ll}\mathrm{a} & 9.11599(16) \AA \\ \mathrm{b} & 13.3173(2) \AA \\ \mathrm{c} & 34.5039(6) \AA \\ \mathrm{V} & 1930.56(7) \AA^{3} \\ \text { Space Group } & P 212121 \\ \text { Z value } & 8 \\ \text { Dcalc } & 1.267 \mathrm{~g} / \mathrm{cm}^{3} \\ \text { Fo00 } & 1696.00 \\ \text { Flack parameter } & -0.01(7)\end{array}$




\section{4-2. syn-Mannich adduct}

Single crystals of syn-3a were obtained by slow evaporation from solution in EtOAc at rt. A suitable crystal was selected and the sample was measured on a Rigaku R-AXIS RAPID diffractometer using graphite monochromated $\mathrm{Cu}-\mathrm{K} \alpha$ radiation. The data were collected at $93 \mathrm{~K}$. Refined structure and crystallographic parameters are summarized in Figure S7 and Table S6. CCDC 1469657 contains the supplementary crystallographic data for syn-3a. Absolute configuration of syn-3a was determined as $2 R, 3 R$ and the absolute configuration of other products $\mathbf{3} \mathbf{g}$, $3 \mathbf{j}$, $\mathbf{3 0}$ and $\mathbf{3 d g}$ were deduced by analogy.

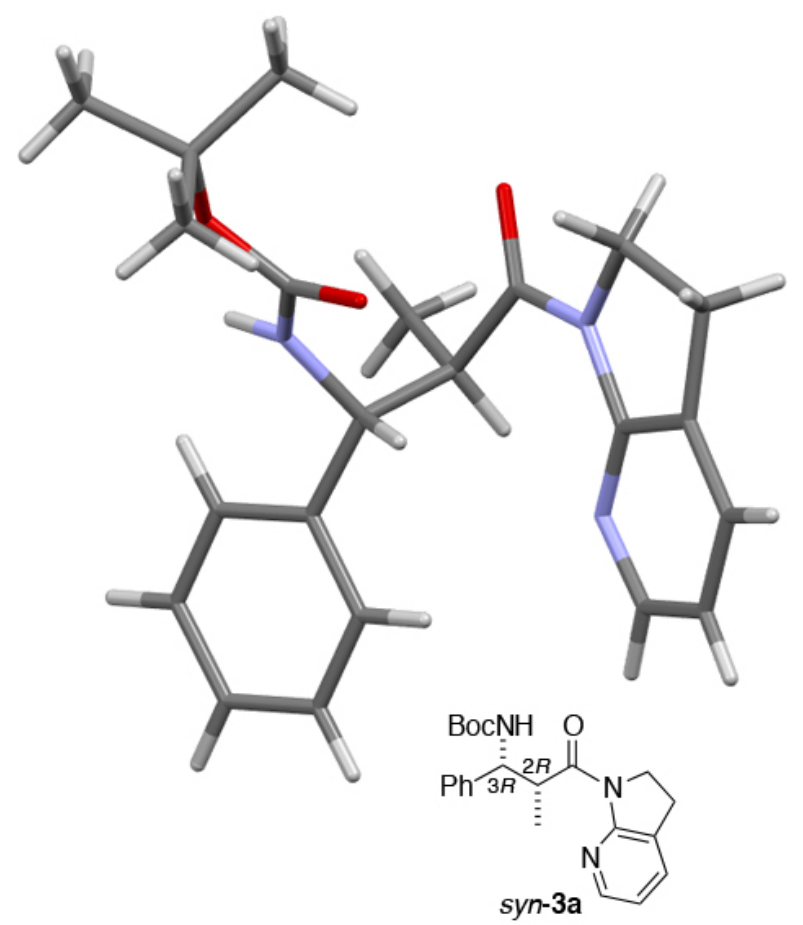

Table S6. Selected crystal data of $s y n-3 \mathbf{a}$. Empirical Formula $\quad \mathrm{C}_{22} \mathrm{H}_{27} \mathrm{O}_{3} \mathrm{~N}_{3}$ Formula Weight $\quad 381.47$ Crystal Color, Habit colorless, prism Crystal Dimensions $\quad 0.200 \times 0.200 \times 0.100 \mathrm{~mm}$ Crystal System orthorhombic Lattice Parameters

$\begin{array}{ll}\mathrm{a} & 9.3255(2) \AA \\ \mathrm{b} & 10.2652(2) \AA \\ \mathrm{c} & 20.1671(4) \AA \\ \mathrm{V} & 1930.56(7) \AA^{3} \\ \text { Space Group } & P 212121 \\ \text { Z value } & 4 \\ \text { Dcalc } & 1.312 \mathrm{~g} / \mathrm{cm}^{3} \\ \text { Fooo } & 816.00 \\ \text { Flack parameter } & -0.03(4)\end{array}$

Figure S7. Structure of syn-3a. Color code; white: hydrogen, gray: carbon, blue: nitrogen, red: oxygen.
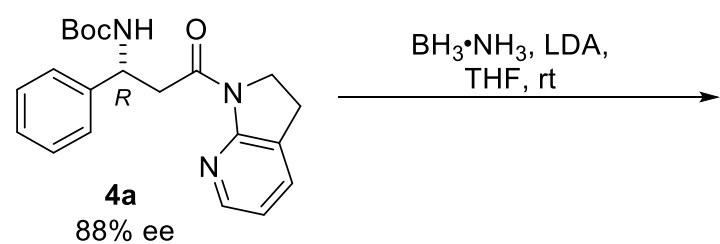

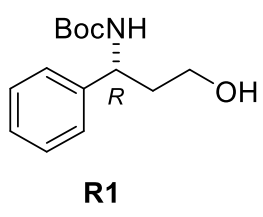

[?] $]_{\mathrm{D}}^{26} 47.0$ (c $0.9, \mathrm{CHCl}_{3}$ )

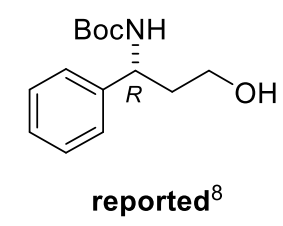

[?] $]_{\mathrm{D}}^{26} 48.6$

(c $1.2, \mathrm{CHCl}_{3}$ ) from $95 \%$ ee sample

Product 4a (88\% ee) was reduced by Myers' protocol $^{7}$ to give $\gamma$-amino alcohol $\mathbf{R} \mathbf{1}$ and its optical rotation was compared to the reported value, ${ }^{8}$ revealing that $R$ configuration at the benzylic position. Thus, absolute configuration of 4 a was determined as $(R)-\mathbf{4 a}$ and the absolute configuration of other products $\mathbf{4 b}, \mathbf{4} \mathbf{g}$ and $\mathbf{4} \mathbf{p}$ were deduced by analogy. The sign of optical rotation of $4 \mathrm{a}, 4 \mathrm{~b}, \mathbf{4 g}$ and $4 \mathrm{p}$ are consistent. 


\section{Transformation of product $3 \mathrm{~g}$}

\section{(2R,3S)-3-Amino-3-(4-fluorophenyl)-2-methylpropanoic acid hydrochloride (5)}

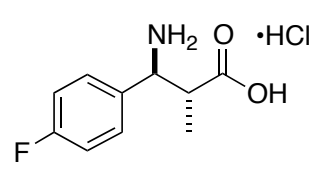

A $20 \mathrm{~mL}$ test tube was charged with $3 \mathrm{~g}(40.0 \mathrm{mg}, 0.1 \mathrm{mmol})$ under air. $6 \mathrm{~N} \mathrm{HCl}$ aq. was subsequently added. The reaction mixture was stirred at $80^{\circ} \mathrm{C}$ for $4 \mathrm{~h}$ before it was cooled to ambient temperature, evaporated and directly purified by flash column chromatography using basic Wakogel ${ }^{\circledR} 50 \mathrm{NH}_{2}\left(\mathrm{CH}_{2} \mathrm{Cl}_{2} / \mathrm{MeOH} 4: 1\right.$, then $0.5 \mathrm{~N} \mathrm{HCl}$ solution in $\left.\mathrm{CH}_{2} \mathrm{Cl}_{2} / \mathrm{MeOH} 1: 1\right)$ to

afford $18 \mathrm{mg}(80 \%)$ of 5 .

White solid, decomposed above $200{ }^{\circ} \mathrm{C} ;{ }^{1} \mathrm{H}$ NMR $\left(400 \mathrm{MHz}, 323 \mathrm{~K}, \mathrm{D}_{2} \mathrm{O}\right): \delta=7.76-7.73(\mathrm{~m}, 2 \mathrm{H}), 7.55-7.50(\mathrm{~m}, 2 \mathrm{H}), 4.84$ $(\mathrm{d}, J=9.6 \mathrm{~Hz}, 1 \mathrm{H}), 3.49-3.41(\mathrm{~m}, 1 \mathrm{H}), 1.35(\mathrm{~d}, J=6.8 \mathrm{~Hz}, 3 \mathrm{H}) ;{ }^{13} \mathrm{C}$ NMR $\left(100 \mathrm{MHz}, 323 \mathrm{~K}, \mathrm{D}_{2} \mathrm{O}\right): \delta$ 180.5, $166.0(\mathrm{~d}, J=244.2$ $\mathrm{Hz}), 133.4(J=2.9 \mathrm{~Hz}), 132.7(\mathrm{~d}, J=8.8 \mathrm{~Hz}), 119.2(\mathrm{~d}, J=21.9 \mathrm{~Hz}), 59.5,46.1,17.3 ;{ }^{19} \mathrm{~F}$ NMR $\left(376 \mathrm{MHz}, 298 \mathrm{~K}, \mathrm{D}_{2} \mathrm{O}\right): \delta=-$ 112.6 (s, 1F); IR (KBr): $\widetilde{v}=3010$ (br), 1605, 1517, 1458, 1407, 1228, $838 \mathrm{~cm}^{-1}$; HRMS (ESI) $\mathrm{m} / z$ calculated for $\mathrm{C}_{10} \mathrm{H}_{13} \mathrm{O}_{2} \mathrm{NF}$ $[\mathrm{M}+\mathrm{H}]^{+}:$198.0925, found: 198.0925; $[\alpha]_{\mathrm{D}}^{26} 7.7(c 0.15, \mathrm{MeOH})$.

\section{tert-Butyl ((1S,2R)-1-(4-fluorophenyl)-2-methyl-3-oxopropyl)carbamate (6)}

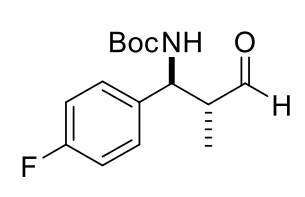

A flame-dried $20 \mathrm{~mL}$ flask was charged with $3 \mathrm{~g}(47.3 \mathrm{mg}, 0.12 \mathrm{mmol})$. After evacuating for 15 min, the tube was backfilled with Ar and anhydrous THF $(1.0 \mathrm{~mL})$ was added. The resulting clear colorless solution was cooled to $-70{ }^{\circ} \mathrm{C}$ and a solution of $\mathrm{LiAlH}_{4}(1.0 \mathrm{M}$ in Et $2 \mathrm{O}, 0.26 \mathrm{~mL}$, $0.24 \mathrm{mmol}, 2.2$ equiv) was slowly added. The reaction mixture was stirred for $45 \mathrm{~min}$ before it was cooled to $-84{ }^{\circ} \mathrm{C}$ using an EtOAc/liquid $\mathrm{N}_{2}$ cooling bath, diluted with anhydrous THF $(5 \mathrm{~mL})$ and carefully quenched under vigorous stirring by the addition of citric acid (1.0 M in THF, $1 \mathrm{~mL})$. Afterwards, the mixture was poured on aqueous citric acid $(0.5 \mathrm{M})$ and extracted three times with diethyl ether. The combined organic extracts were dried over $\mathrm{Mg}_{2} \mathrm{SO}_{4}$, filtered and evaporated to give the crude aldehyde, which was directly purified by quickly passing through a short pad of silica between two thin layers of Celite $\left(\mathrm{CH}_{2} \mathrm{Cl}_{2} / \mathrm{Et}_{2} \mathrm{O}, 30: 1\right)$. After evaporation, $33.3 \mathrm{mg}(83 \%)$ of 6 was isolated.

White solid, m.p.: $131-133{ }^{\circ} \mathrm{C} .{ }^{1} \mathrm{H}$ NMR $\left(500 \mathrm{MHz}, 298 \mathrm{~K}, \mathrm{CDCl}_{3}\right): \delta=9.65$ (d, J = $\left.2.5 \mathrm{~Hz}, 1 \mathrm{H}\right), 7.27-7.24$ (m, 2H), 7.07$7.04(\mathrm{~m}, 2 \mathrm{H}), 5.21(\mathrm{brs}, 1 \mathrm{H}), 4.87(\mathrm{brs}, 1 \mathrm{H}), 2.82(\mathrm{brs}, 1 \mathrm{H}), 1.42(\mathrm{~s}, 9 \mathrm{H}), 1.07(\mathrm{~d}, J=6.5 \mathrm{~Hz}, 3 \mathrm{H}) ;{ }^{13} \mathrm{C} \mathrm{NMR}(125 \mathrm{MHz}, 298 \mathrm{~K}$, $\left.\mathrm{CDCl}_{3}\right): \delta 203.1,162.1(\mathrm{~d}, J=244.6 \mathrm{~Hz}), 155.1,135.9,128.4(\mathrm{~d}, J=8.1 \mathrm{~Hz}), 115.6(\mathrm{~d}, J=20.8 \mathrm{~Hz}), 80.1,55.2,52.0,28.3,11.9$; ${ }^{19} \mathrm{~F}$ NMR (376 MHz, $\left.300 \mathrm{~K}, \mathrm{CDCl}_{3}\right): \delta=-116.9$ (s, 1F); IR (KBr): $\widetilde{v}=3362,2975,2814,2721,1723,1680,1509,1223 \mathrm{~cm}^{-1}$; HRMS (ESI) $m / z$ calculated for $\mathrm{C}_{15} \mathrm{H}_{21} \mathrm{O}_{3} \mathrm{NF}[\mathrm{M}+\mathrm{H}]^{+}: 282.1500$, found: 282.1498; $[\alpha]_{\mathrm{D}}^{25}-11.4$ (c 0.07, $\mathrm{CHCl}_{3}$ ).

\section{tert-Butyl ((1S,2R)-1-(4-fluorophenyl)-2-methyl-3-oxo-3-phenylpropyl)carbamate (7)}

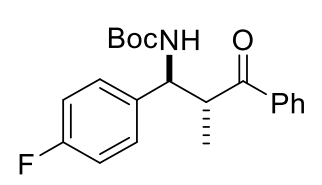

A flame-dried $20 \mathrm{~mL}$ test tube was charged with $3 \mathrm{~g}$ ( $23 \mathrm{mg}, 0.06 \mathrm{mmol})$. After evacuating for 15 min, the tube was backfilled with Ar and anhydrous THF $(1.0 \mathrm{~mL})$ was added. Subsequently, phenylmagnesium bromide (1.0 M in THF, $0.3 \mathrm{~mL}, 0.3 \mathrm{mmol}, 5.0$ equiv) was added at $0{ }^{\circ} \mathrm{C}$. The reaction mixture was stirred at ambient temperature for $45 \mathrm{~min}$ before it was cooled to $0{ }^{\circ} \mathrm{C}$ and quenched by the addition of sat. $\mathrm{NH}_{4} \mathrm{Cl}$ aq. The mixture was diluted with water and extracted three times with EtOAc; the combined organic extracts were dried over $\mathrm{Mg}_{2} \mathrm{SO}_{4}$, filtered and evaporated to afford $13 \mathrm{mg}$ (64\%) of 7 after purification by flash column chromatography (hexane/EtOAc, 10:1).

White solid, m.p.: $116-117^{\circ} \mathrm{C} ;{ }^{1} \mathrm{H}$ NMR $\left(400 \mathrm{MHz}, 300 \mathrm{~K}, \mathrm{CDCl}_{3}\right): \delta=7.77-7.75(\mathrm{~m}, 2 \mathrm{H}), 7.55-7.51(\mathrm{~m}, 1 \mathrm{H}), 7.42-7.38(\mathrm{~m}$, 2H), 7.25-7.22 (m, 2H), 6.95-6.90 (m, 2H), 6.24 (brs, 1H), 5.01-4.97 (m, 1H), 4.04-4.01 (m, 1H), 1.45 (s, 9H), 1.37 (d, J = 7.2 $\mathrm{Hz}, 3 \mathrm{H}) ;{ }^{13} \mathrm{C}$ NMR $\left(100 \mathrm{MHz}, 300 \mathrm{~K}, \mathrm{CDCl}_{3}\right): \delta 204.2,161.9$ (d, J = 244.2 Hz), 155.8, 137.6 (d, J = 2.9 Hz), 136.8, 133.3, 128.7, $128.2,127.9(\mathrm{~d}, J=8.0 \mathrm{~Hz}), 115.2(\mathrm{~d}, J=21.1 \mathrm{~Hz}), 79.5,57.2,45.1,28.4,16.4 ;{ }^{19} \mathrm{~F}$ NMR $\left(376 \mathrm{MHz}, 298 \mathrm{~K}, \mathrm{CDCl}_{3}\right): \delta=-115.9$ (s, 1F); IR (KBr): $\widetilde{v}=3429,3064,2978,2932,2875,1700,1604,1509,1227,1161,971 \mathrm{~cm}^{-1}$; HRMS (ESI) $\mathrm{m} / \mathrm{z}$ calculated for $\mathrm{C}_{21} \mathrm{H}_{24} \mathrm{O}_{3} \mathrm{NFNa}^{+}[\mathrm{M}+\mathrm{Na}]^{+}:$380.1632, found: 380.1633; [ $\left.\alpha\right]_{\mathrm{D}^{25}-21.1}\left(\mathrm{c} 0.15, \mathrm{CHCl}_{3}\right)$.

\section{tert-Butyl ((1S,2R)-1-(4-fluorophenyl)-3-hydroxy-2-methylpropyl)carbamate (8)}

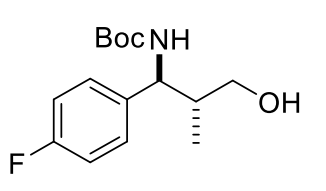

To a solution of lithium diisopropylamide (1.0 M in $n$-hexane/THF (5.4:1), $0.397 \mathrm{~mL}, 0.39 \mathrm{mmol}$, 3.9 equiv.) in anhydrous THF $(0.5 \mathrm{~mL})$ was added borane-ammonia complex (12.6 mg, 0.40 mmol, 4.0 equiv.) at $0{ }^{\circ} \mathrm{C}$. After stirring for $10 \mathrm{~min}$ at the same temperature, the reaction mixture 
was stirred at room temperature for another $10 \mathrm{~min}$ before it was cooled back to $0{ }^{\circ} \mathrm{C}$. A solution of $3 \mathrm{~g}(40.6 \mathrm{mg}, 0.1$ $\mathrm{mmol})$ in anhydrous THF $(0.5 \mathrm{~mL})$ was subsequently added. After stirring for $30 \mathrm{~min}$, the reaction mixture was warmed to ambient temperature and stirred for another $30 \mathrm{~min}$ before it was finally quenched by the addition of sat. $\mathrm{NH}_{4} \mathrm{Cl}$ aq. The mixture was diluted with water and extracted three times with EtOAc; the combined organic extracts were treated with aqueous $\mathrm{NaOH}(1 \mathrm{M})$ solution and stirred for $30 \mathrm{~min}$. Then, the layers were separated and the aqueous layer was extracted twice with EtOAc; the combined organic extracts were dried over $\mathrm{Mg}_{2} \mathrm{SO}_{4}$, filtered and evaporated to afford $29 \mathrm{mg}$ ( $84 \%$ ) of 8 after purification by flash column chromatography (hexane/EtOAc, 5:1).

White solid, m.p.: $113-114{ }^{\circ} \mathrm{C}$; ${ }^{1} \mathrm{H}$ NMR $\left(500 \mathrm{MHz}, 298 \mathrm{~K}, \mathrm{CD}_{3} \mathrm{CN}\right): \delta=7.31-7.28(\mathrm{~m}, 2 \mathrm{H}), 7.09-7.05(\mathrm{~m}, 2 \mathrm{H}), 6.03$ (brs, 1H), 4.51 (brs, 1H), 3.52-3.42 (m, 2H), 2.86 (brs, 1H), 1.89 (br, 1H), 1.37 (s, 9H), 0.84 (d, J = 7.0 Hz, 3H); ${ }^{13} \mathrm{C}$ NMR (125 $\left.\mathrm{MHz}, 298 \mathrm{~K}, \mathrm{CD}_{3} \mathrm{CN}\right): \delta 163.8$ (d, J = 241.0 Hz), 156.8, 140.1, 129.8 (d, J = 8.1 Hz), 115.8 (d, J = 20.8 Hz), 79.6, 64.7, 58.6, 41.3, 28.7, 15.1; ${ }^{19} \mathrm{~F}$ NMR (376 MHz, $\left.298 \mathrm{~K}, \mathrm{CDCl}_{3}\right): \delta=-115.9$ (s, 1F); IR (KBr): $\widetilde{v}=3362(\mathrm{br}), 2977,2935,2880,1687,1509$, $1366,1225,1170,843 \mathrm{~cm}^{-1}$; HRMS (ESI) $\mathrm{m} / z$ calculated for $\mathrm{C}_{15} \mathrm{H}_{22} \mathrm{O}_{3} \mathrm{NFNa}^{+}[\mathrm{M}+\mathrm{Na}]^{+}: 306.1476$, found: $306.1479 ;[\alpha]_{\mathrm{D}^{25}-}$ 38.9 ( c 0.53, $\mathrm{MeOH})$. 
1. Fulmer, G. R. et al. Organometallics 2010, 29, 2176.

2. (a) Pearson, S. E.; Nandan, S. Synthesis 2005, 2503. (b) Darout, E. et al. WO2010/128414 A1, 2010.

3. Jössang-Yanagida, A.; Gansser, C. J. Heterocyclic Chem. 1978, 15, 249.

4. (a) Kanazawa, A. M.; Denis, J.-N.; Greene, A. E. J. Org. Chem. 1994, 59, 1238. (b) Love, B. E.; Raje, P. S.; Williams II, T. C. Synlett 1994, 493. (c) Wenzel, A. G.; Jacobsen, E. N. J. Am. Chem. Soc. 2002, 124, 12964. (d) Mbofana, C. T.; Miller, S. J. J. Am. Chem. Soc. 2014, 136, 3285. (e) Goodman, C. G.; Do, D. T.; Johnson, J. S. Org. Lett. 2013, 15, 2446.

5. Weidner, K.; Kumagai, N.; Shibasaki, M. Angew. Chem., Int. Ed. 2014, 53, 6150.

6. The occurance of rotamers is well documented for similar compounds, see for example: Peddie, V.; Abell, A. D. Helv. Chim. Acta 2012, 95, 2460.

7. Myers, A. G.; Yang, B. H.; Chen, H.; McKinstry, L; Kopecky, D. J.; Gleason, J. L. J. Am. Chem. Soc. 1997, 119, 6496.

8. Hatano, M.; Moriyama, K.; Maki, T.; Ishihara, K. Angew. Chem., Int. Ed. 2010, 49, 3823. 

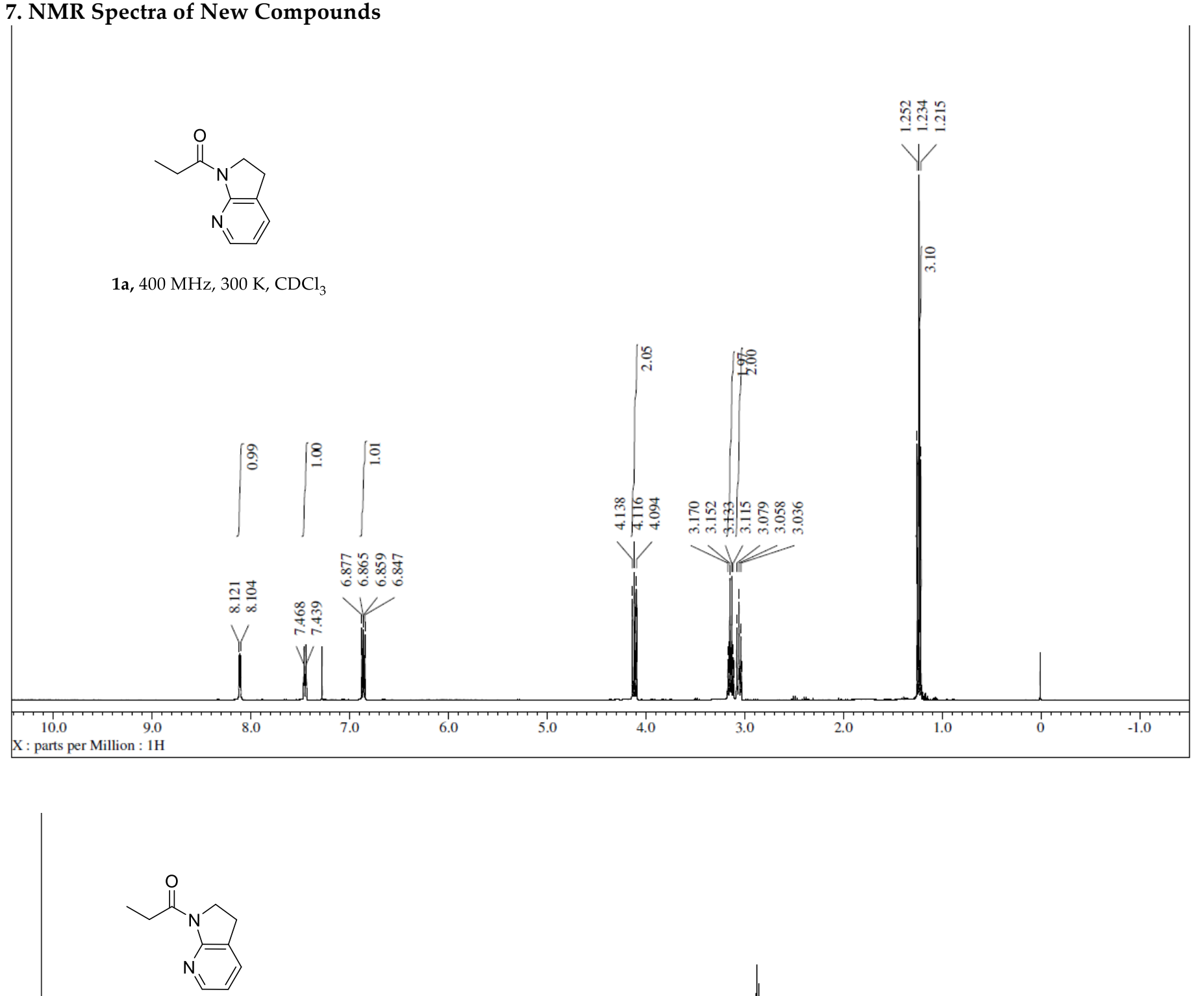

1a, $100 \mathrm{MHz}, 300 \mathrm{~K}, \mathrm{CDCl}_{3}$

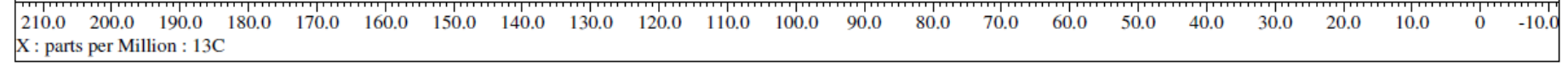


<smiles>CCC(=O)N1CCCc2cccnc21</smiles>

$\mathbf{1 b}, 400 \mathrm{MHz}, 300 \mathrm{~K}, \mathrm{CDCl}_{3}$

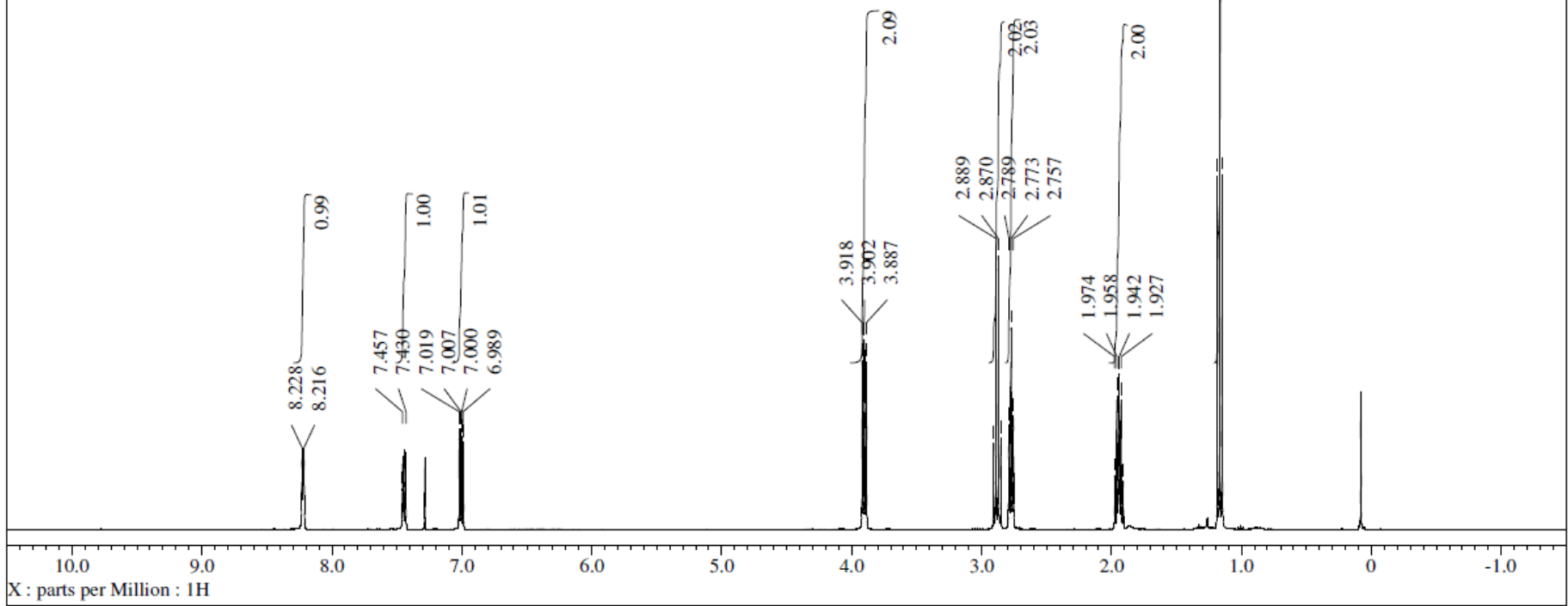<smiles>CCC(=O)N1CCCc2cccnc21</smiles>

1b, $100 \mathrm{MHz}, 300 \mathrm{~K}, \mathrm{CDCl}_{3}$
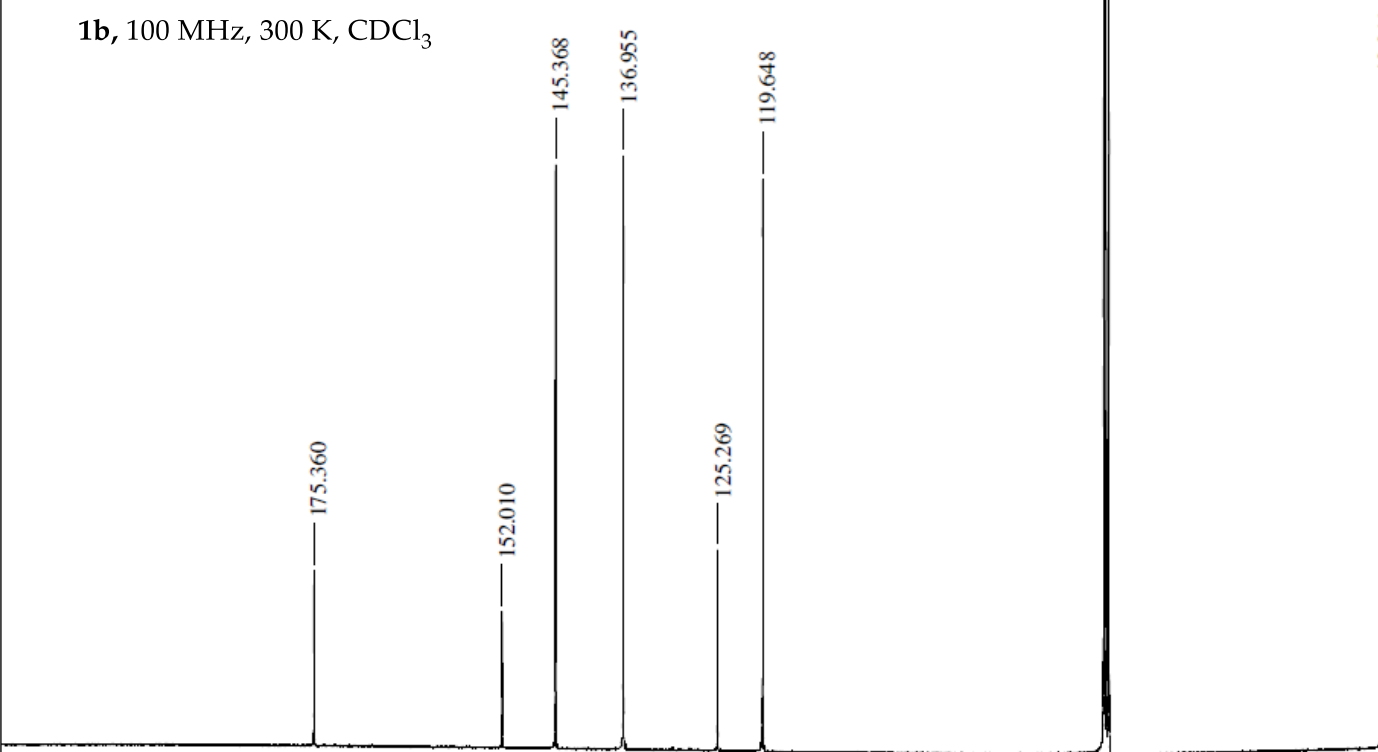

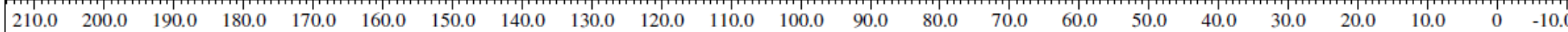
X : parts per Million : $13 \mathrm{C}$ 
<smiles>CCC(=O)N1CCCCc2cccnc21</smiles>

1c, $400 \mathrm{MHz}, 343 \mathrm{~K}, \mathrm{CD}_{3} \mathrm{CN}$
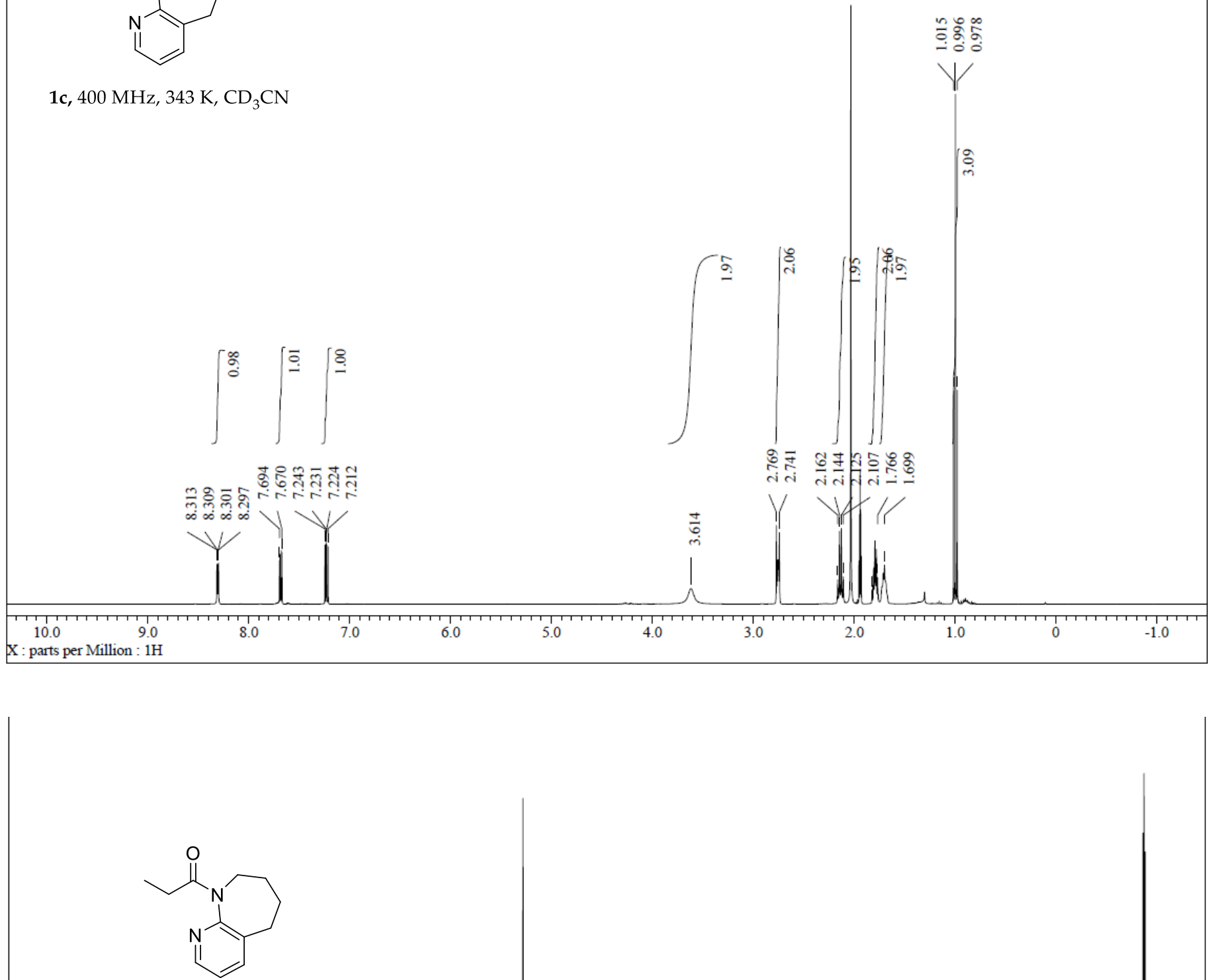

1c, $100 \mathrm{MHz}, 343 \mathrm{~K}, \mathrm{CD}_{3} \mathrm{CN}$ 
<smiles>CCCC(=O)N1CCc2cccnc21</smiles>

1d, $400 \mathrm{MHz}, 300 \mathrm{~K}, \mathrm{CDCl}_{3}$

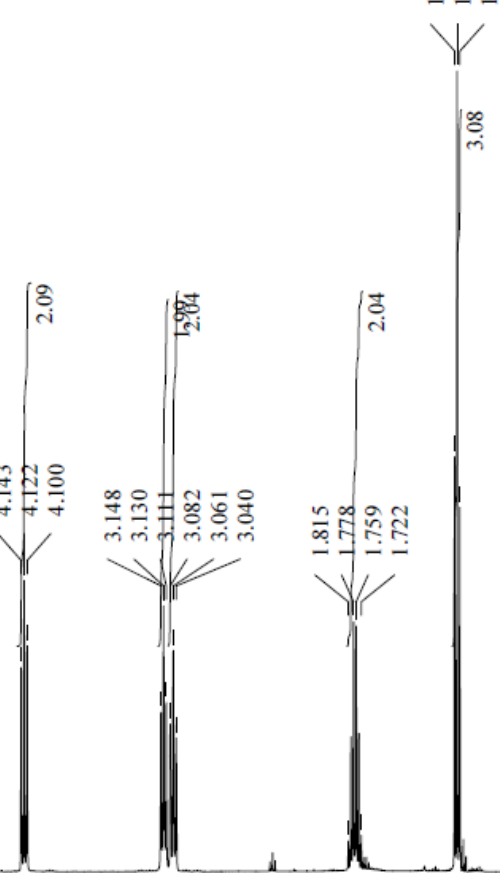

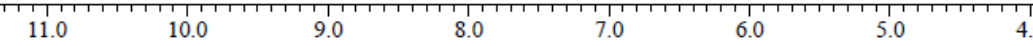

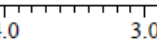

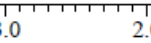

X: parts per Million : $1 \mathrm{H}$

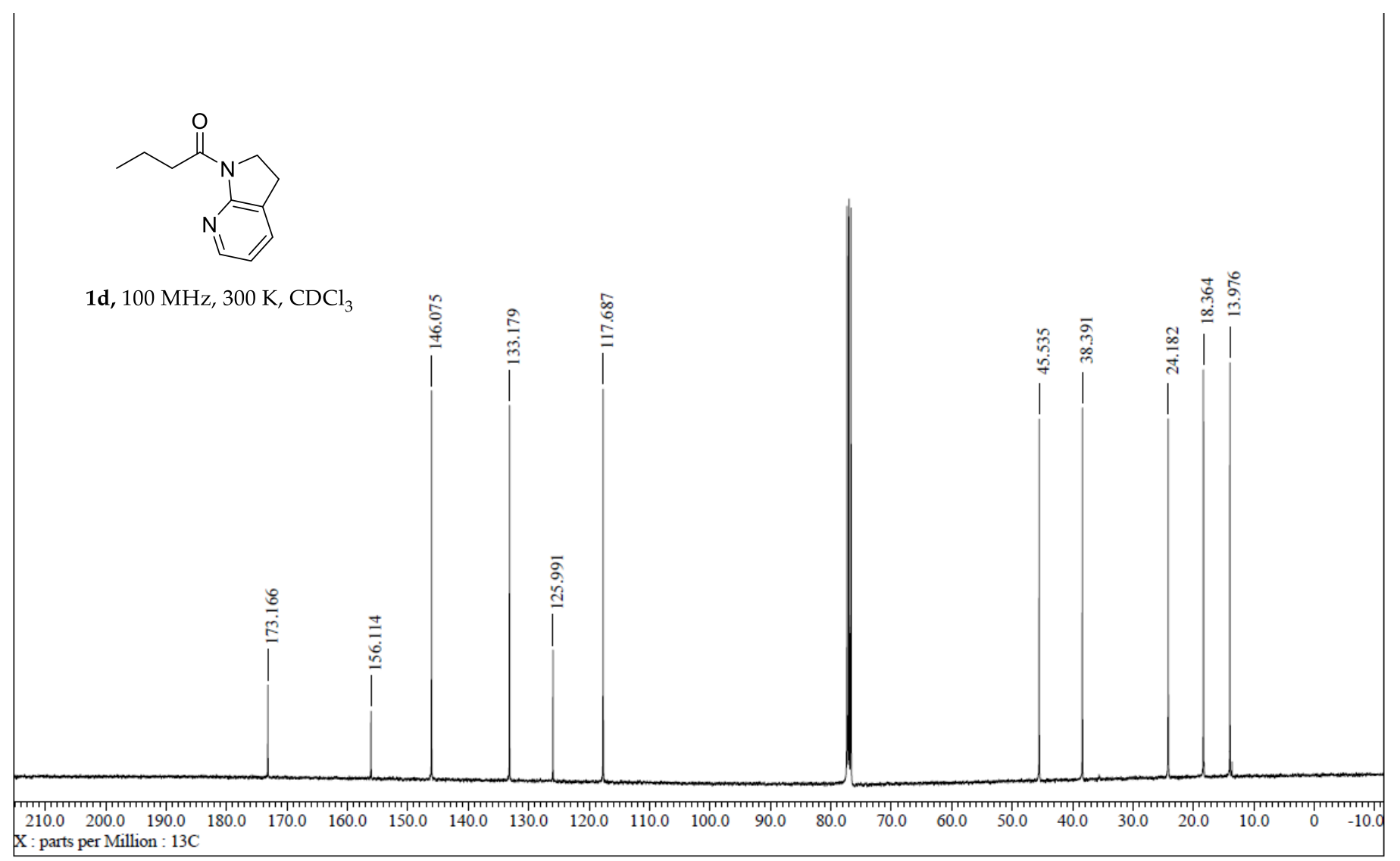




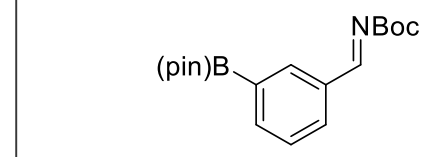

2k, $400 \mathrm{MHz}, 300 \mathrm{~K}, \mathrm{CDCl}_{3}$

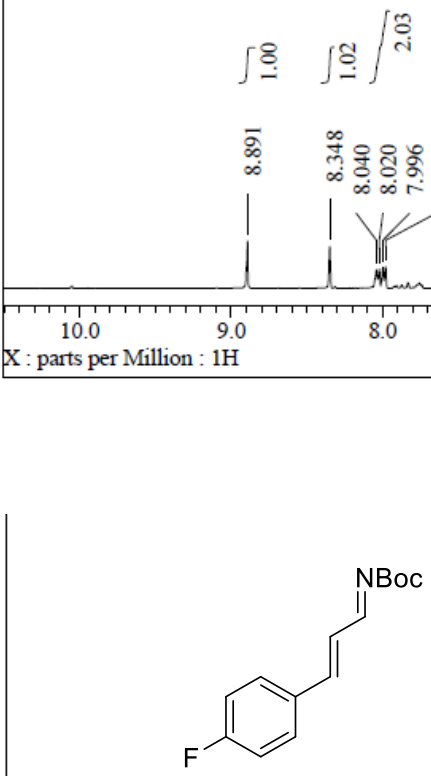

2n, $400 \mathrm{MHz}, 300 \mathrm{~K}, \mathrm{CDCl}_{3}$

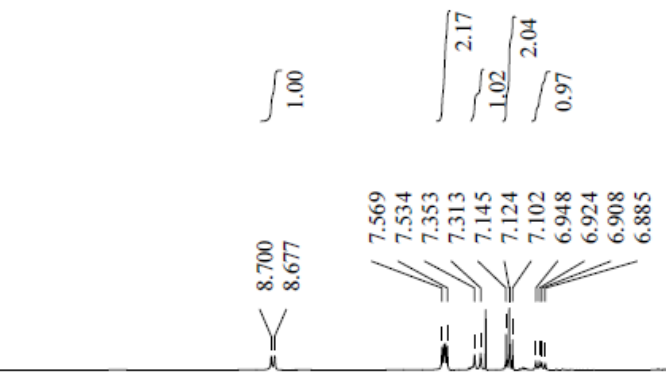

$\begin{array}{ll}10.0 & 9.0\end{array}$

8.0

7.0

6.0

5.0

$\begin{array}{lllll}1 & 1 & 1\end{array}$

$\begin{array}{ll}4.0 & 3.0\end{array}$

2.0

1.0

0

$-1.0$

X : parts per Million : $1 \mathrm{H}$ 
Supporting Information

Direct Catalytic Asymmetric Mannich-Type Reaction of Alkylamides<smiles>C[C@H](C(=O)N1CCc2cccnc21)C(NC(=O)OCc1ccccc1)c1ccccc1</smiles>

anti-3a, $400 \mathrm{MHz}, 323 \mathrm{~K}, \mathrm{CD}_{3} \mathrm{CN}$

(a)

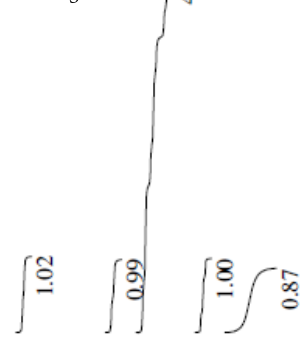

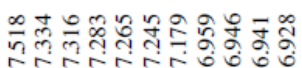
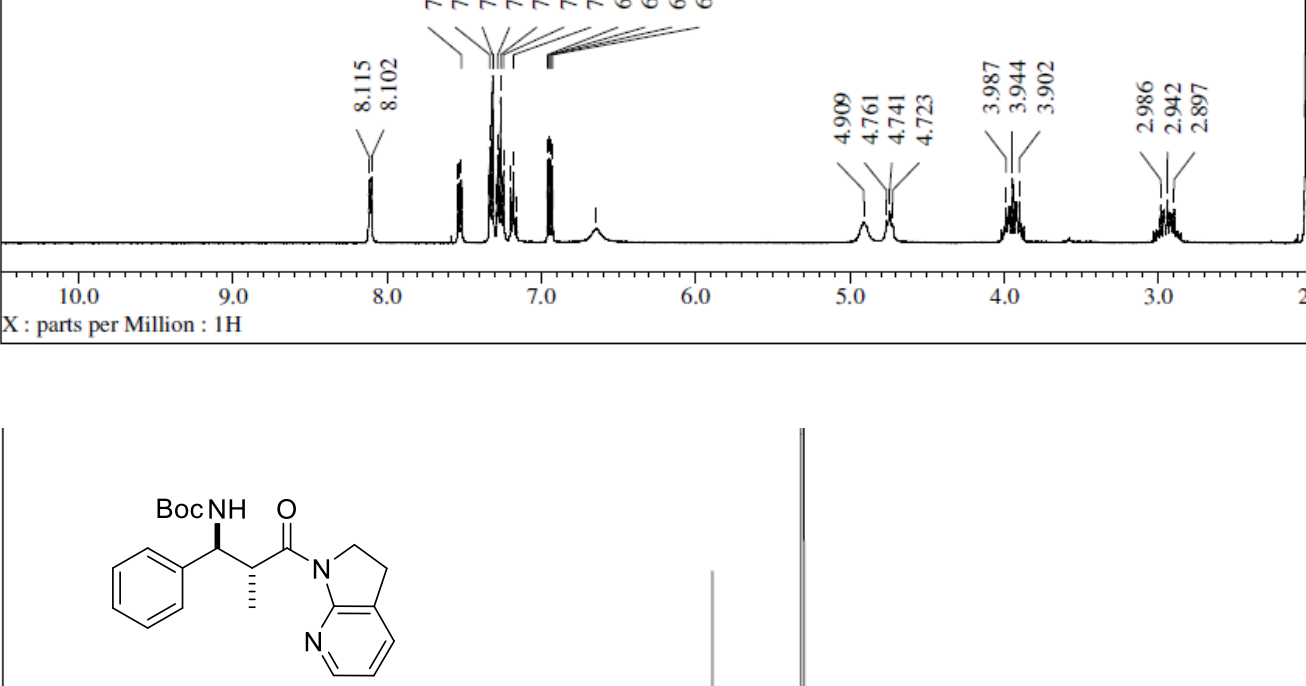

anti-3a, $100 \mathrm{MHz}, 323 \mathrm{~K}, \mathrm{CD}_{3} \mathrm{CN}$

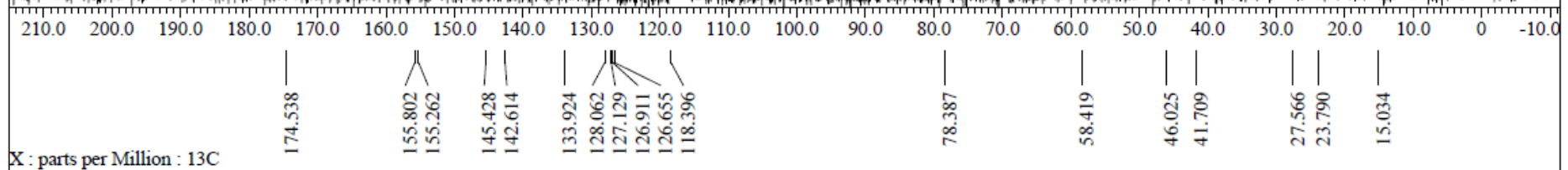



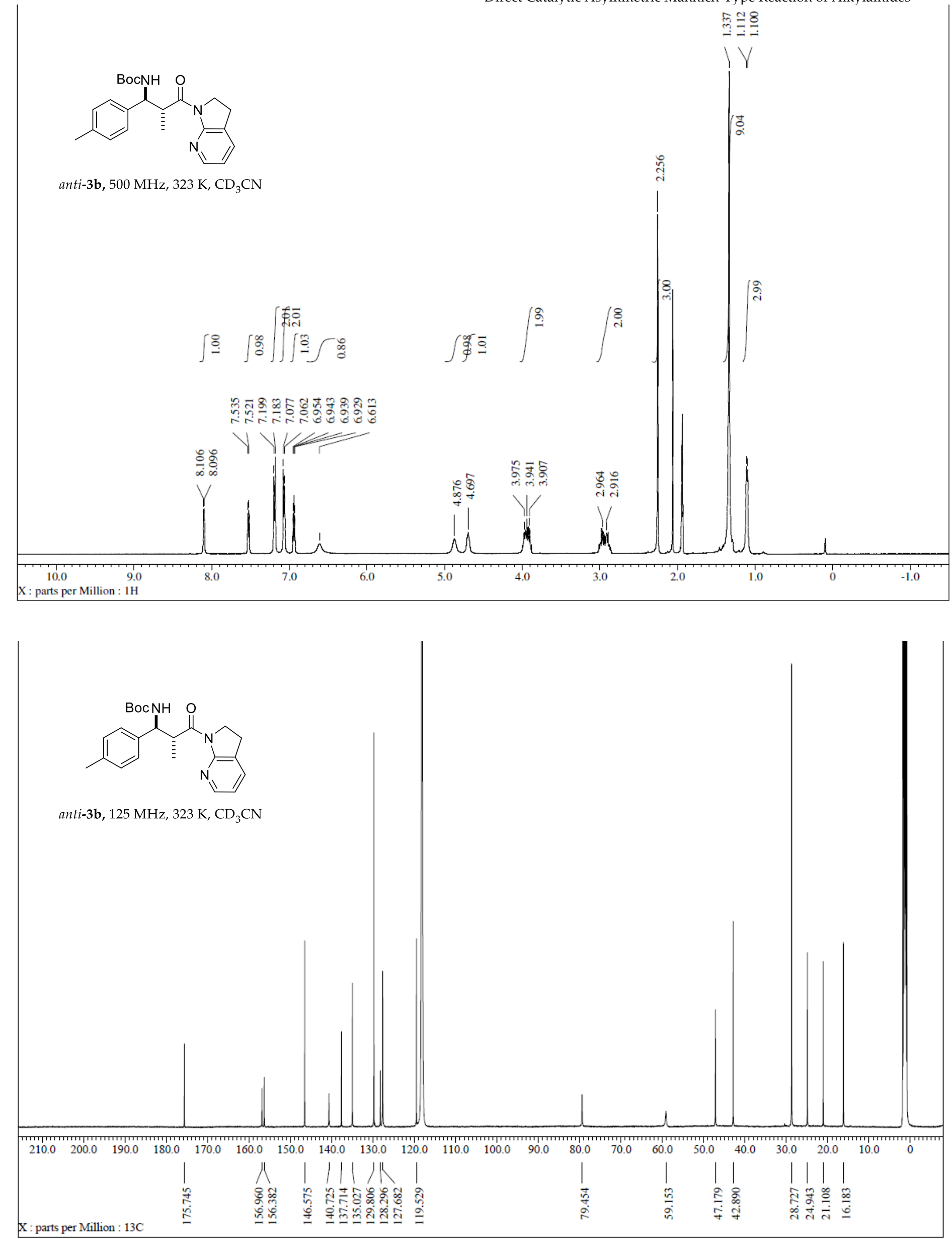
Supporting Information

Direct Catalytic Asymmetric Mannich-Type Reaction of Alkylamides<smiles>COc1cccc(C(NC(=O)OCc2ccccc2)[C@H](C)C(=O)N2CCc3cccnc32)c1</smiles>

anti-3c, $500 \mathrm{MHz}, 323 \mathrm{~K}, \mathrm{CD}_{3} \mathrm{CN}$
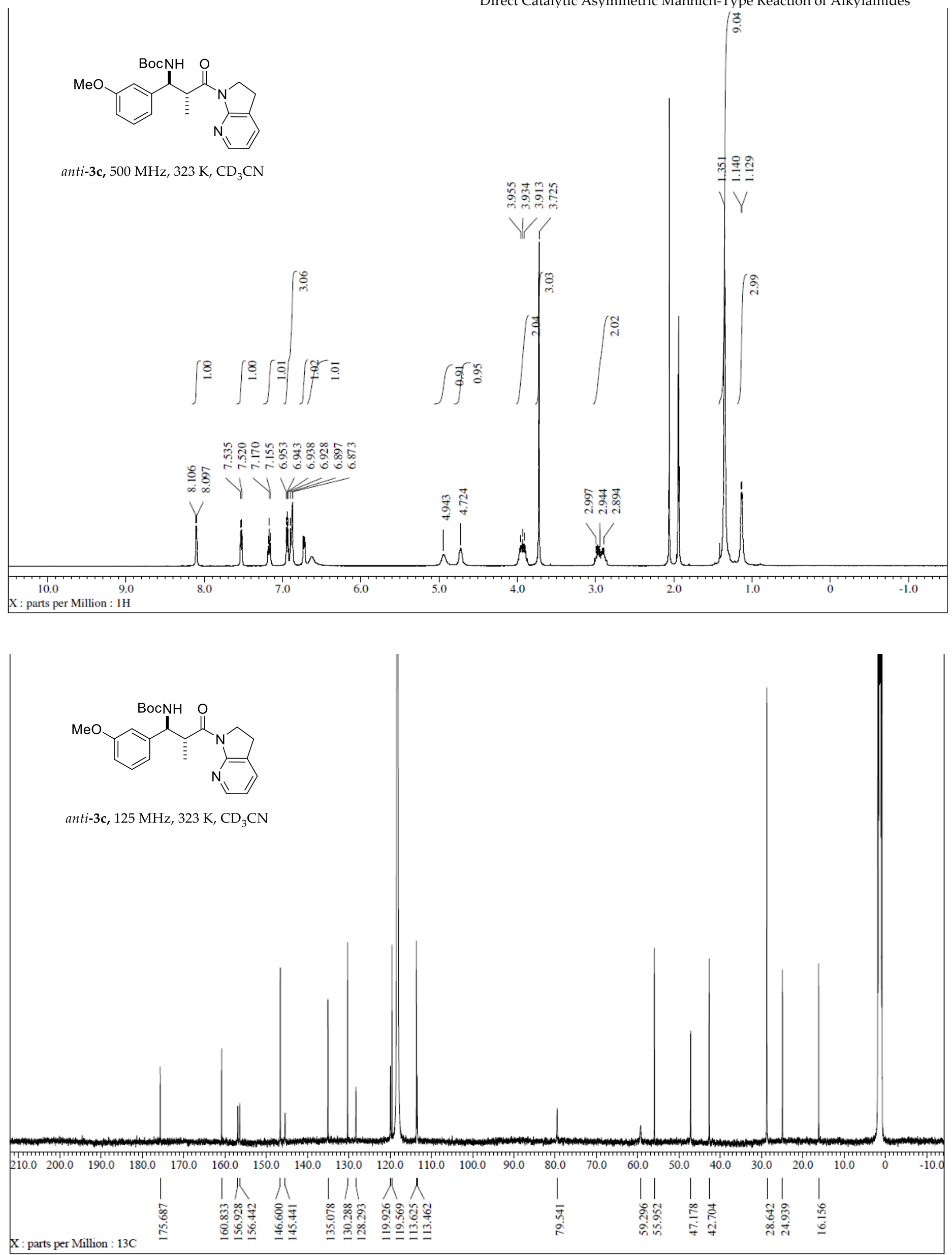
<smiles>COc1ccc(C(NC(=O)OC(C)(C)C)[C@H](C)C(=O)N2CCc3cccnc32)cc1</smiles>

anti-3d, $500 \mathrm{MHz}, 323 \mathrm{~K}, \mathrm{CD}_{3} \mathrm{CN}$

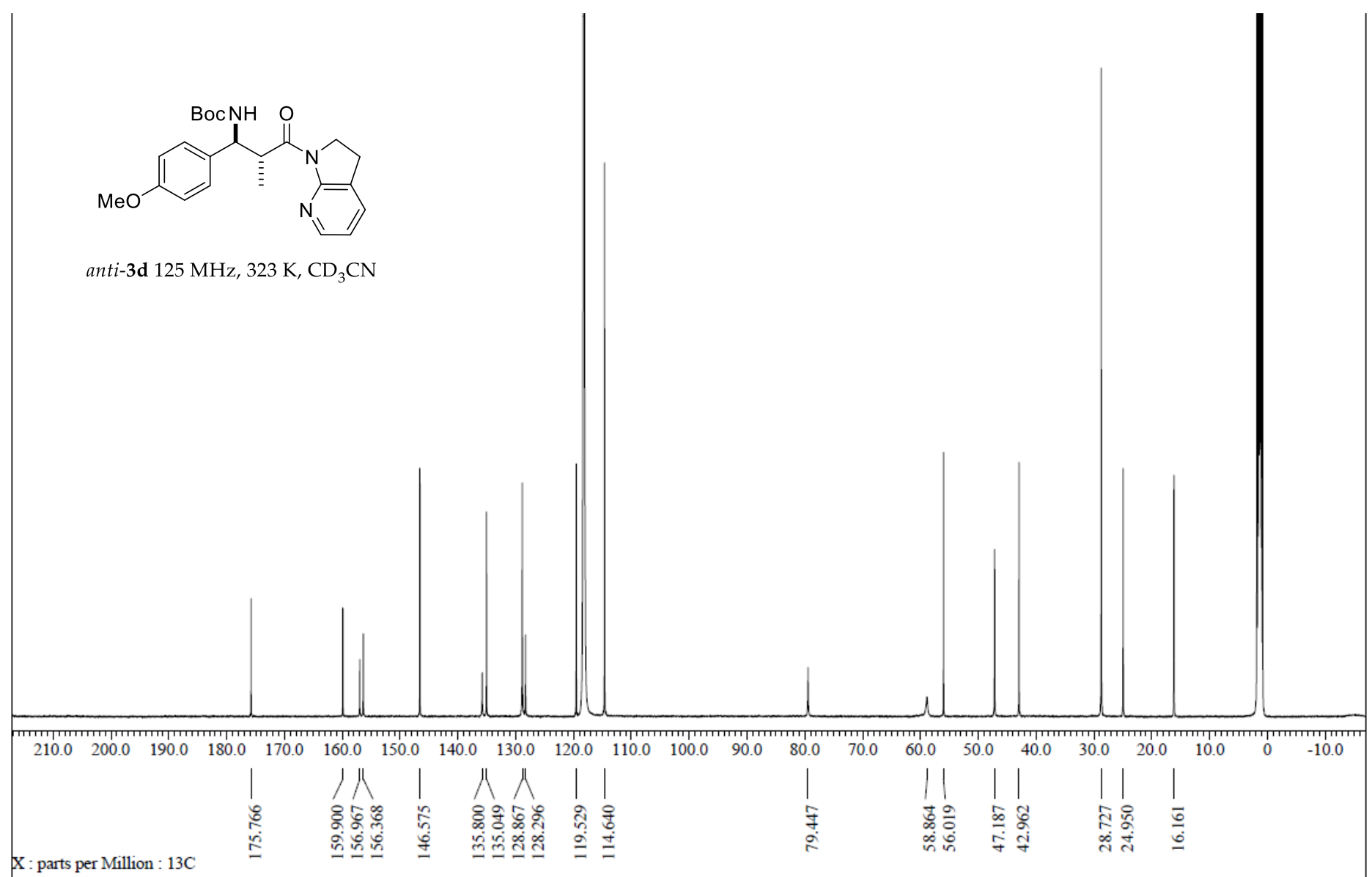

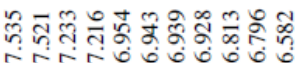
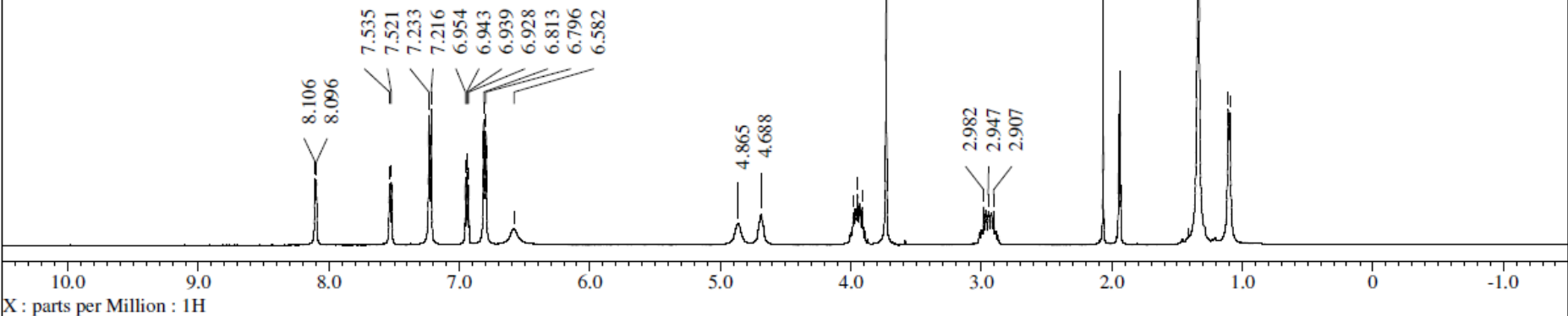
Supporting Information

Direct Catalytic Asymmetric Mannich-Type Reaction of Alkylamides<smiles>COC(=O)N[C@H](c1ccccc1F)C(C)C(=O)N1CCc2cccnc21</smiles>

anti-3e, $400 \mathrm{MHz}, 323 \mathrm{~K}, \mathrm{CD}_{3} \mathrm{CN}$

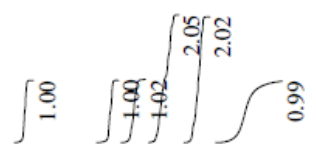

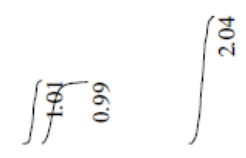
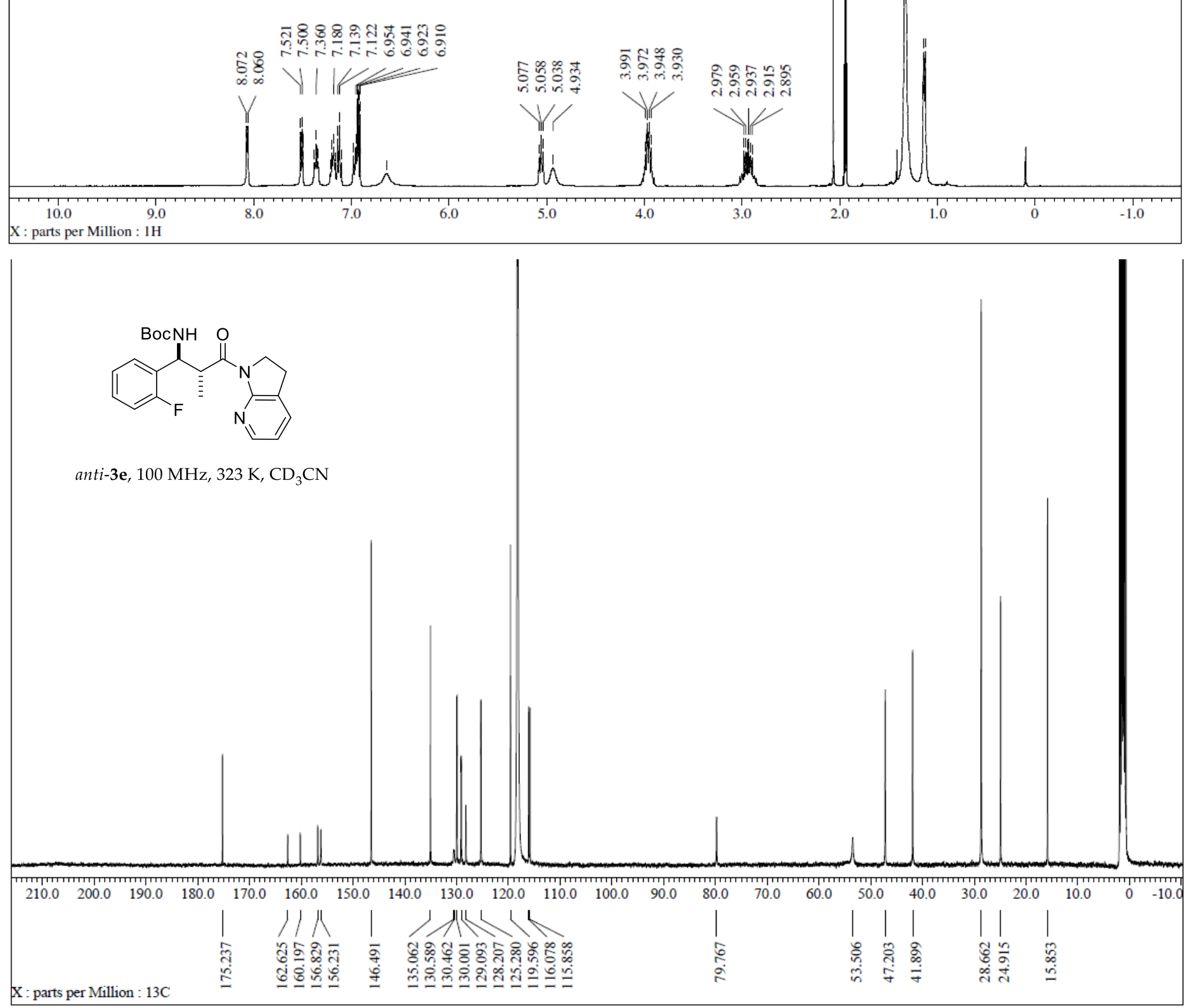

Page S36 of S59 


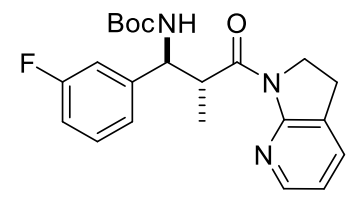

anti-3f, $400 \mathrm{MHz}, 323 \mathrm{~K}, \mathrm{CD}_{3} \mathrm{CN}$

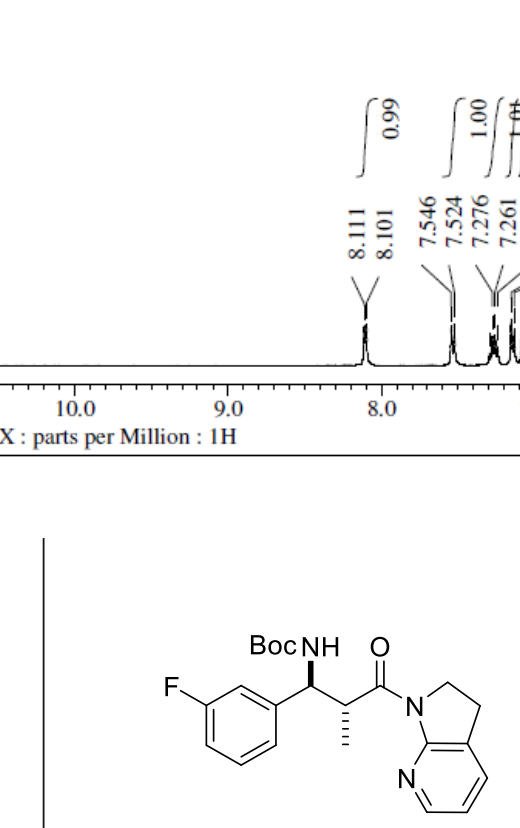

anti-3f, $100 \mathrm{MHz}, 323 \mathrm{~K}, \mathrm{CD}_{3} \mathrm{CN}$

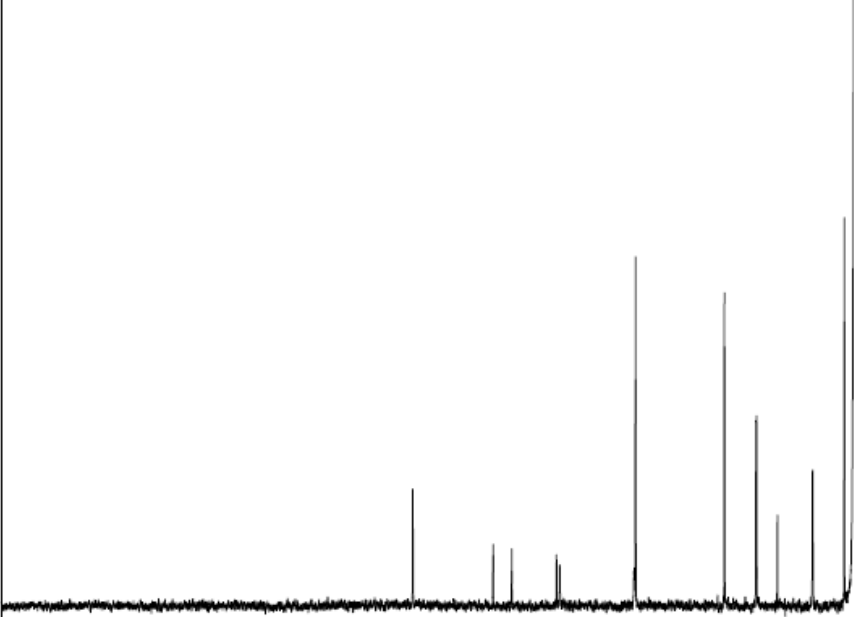

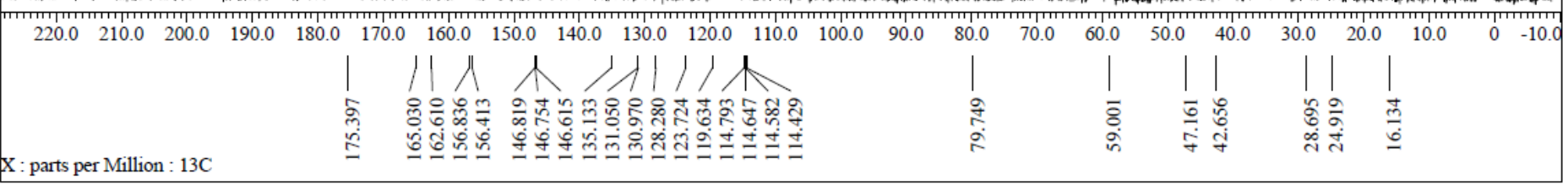




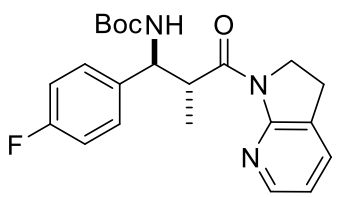

anti-3g, $400 \mathrm{MHz}, 323 \mathrm{~K}, \mathrm{CD}_{3} \mathrm{CN}$<smiles>CCCCC(C)CC</smiles>

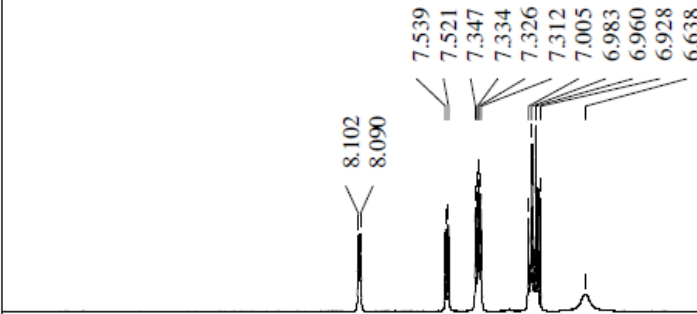

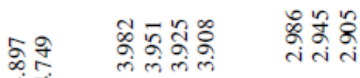

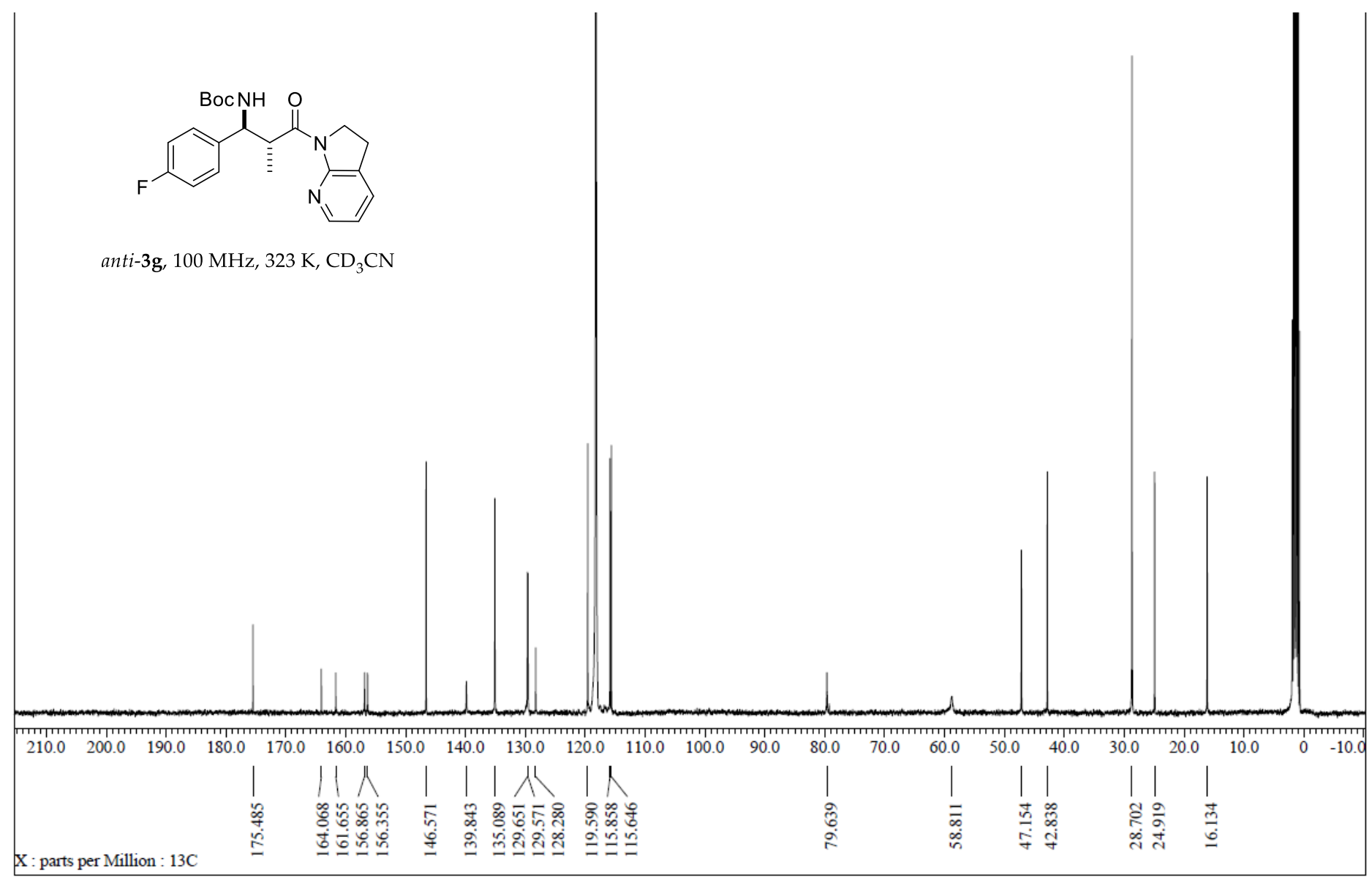

anti-3g, $100 \mathrm{MHz}, 323 \mathrm{~K}, \mathrm{CD}_{3} \mathrm{CN}$ 
<smiles>C[C@H](C(=O)N1CCc2cccnc21)C(NC(=O)OCc1ccccc1)c1cccc([N+](=O)[O-])c1</smiles>

anti-3h, $400 \mathrm{MHz}, 323 \mathrm{~K}, \mathrm{CD}_{3} \mathrm{CN}$
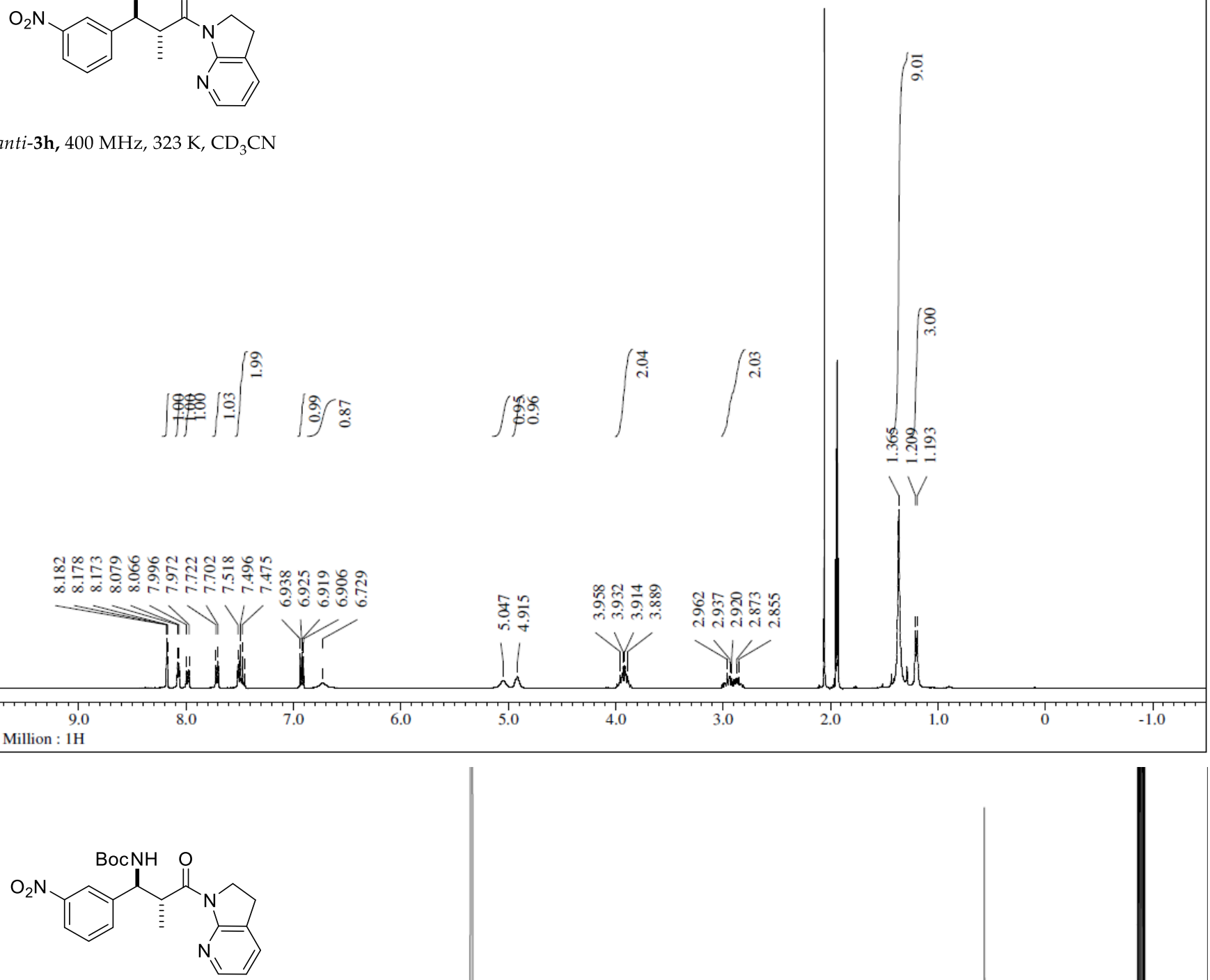

anti-3h, $100 \mathrm{MHz}, 323 \mathrm{~K}, \mathrm{CD}_{3} \mathrm{CN}$

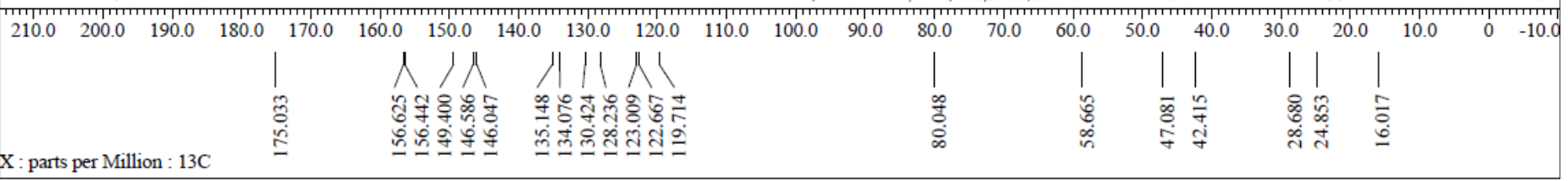


<smiles>C[C@H](C(=O)N1CCc2cccnc21)C(NC(=O)OCc1ccccc1)c1cccc(Br)c1</smiles>
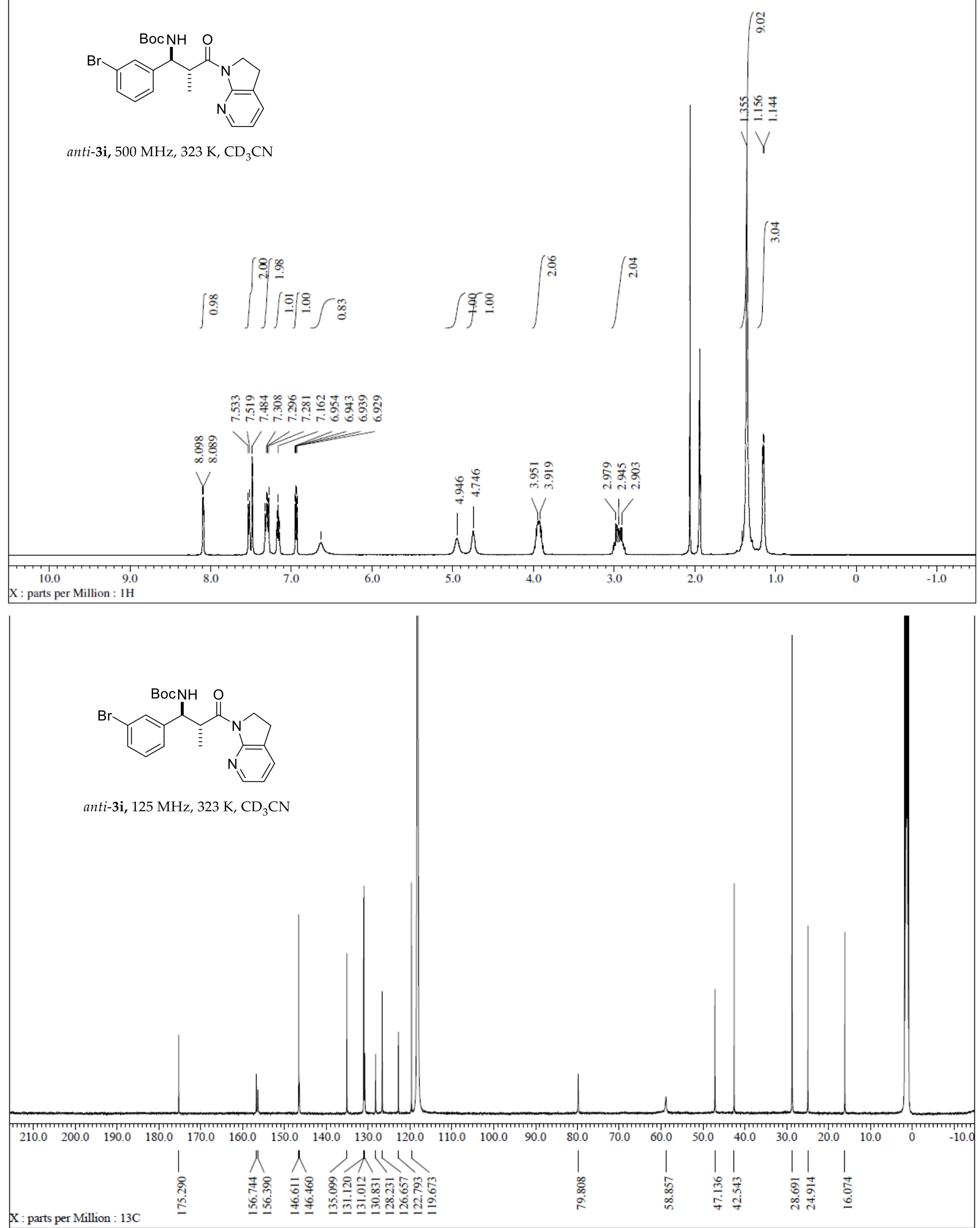


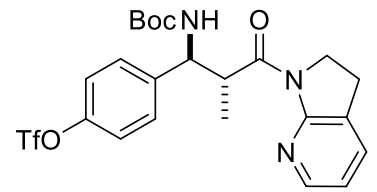

anti-3j, $400 \mathrm{MHz}, 323 \mathrm{~K}, \mathrm{CD}_{3} \mathrm{CN}$
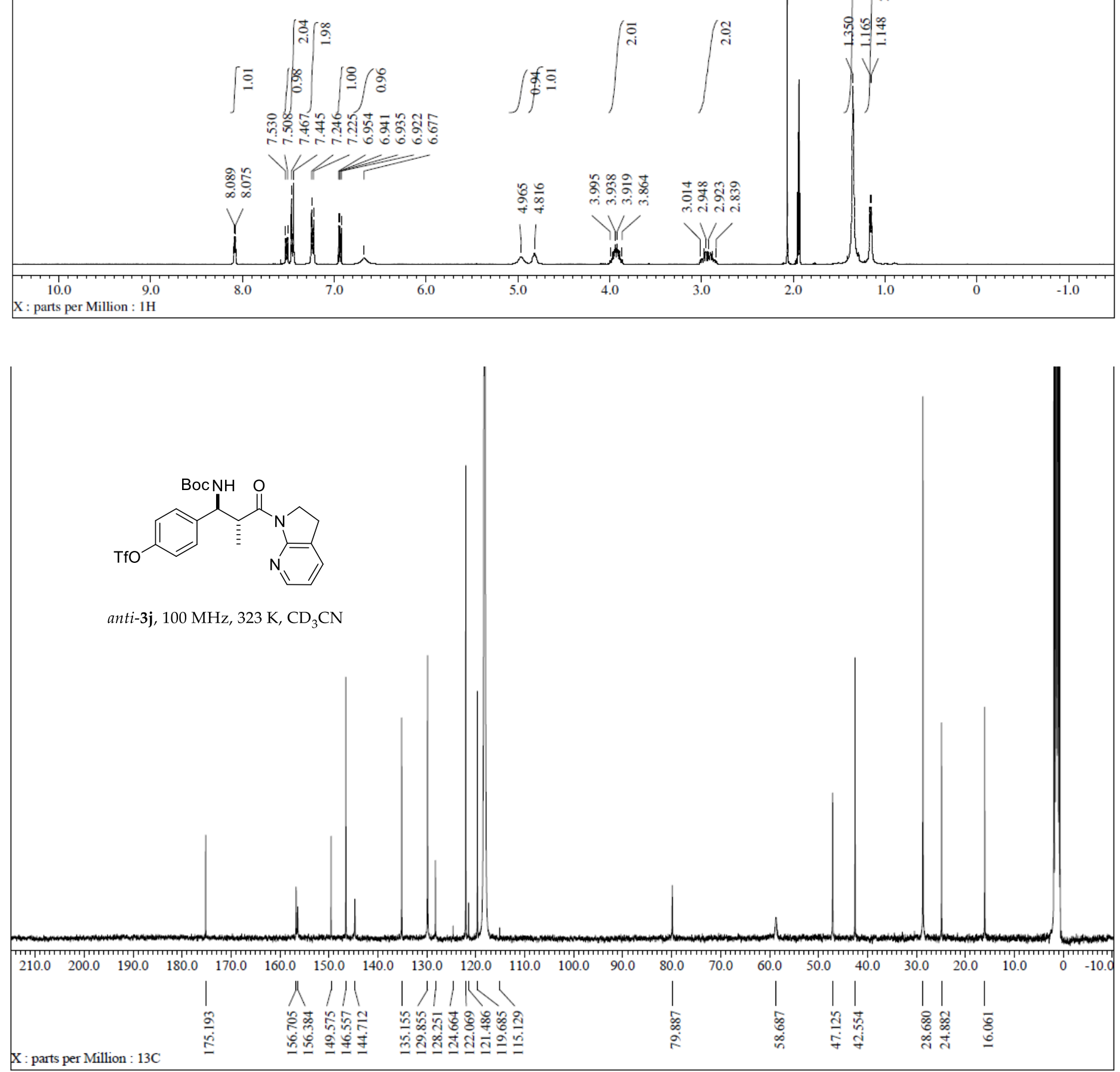


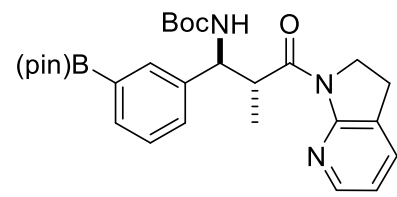

anti-3k, $500 \mathrm{MHz}, 323 \mathrm{~K}, \mathrm{CD}_{3} \mathrm{CN}$
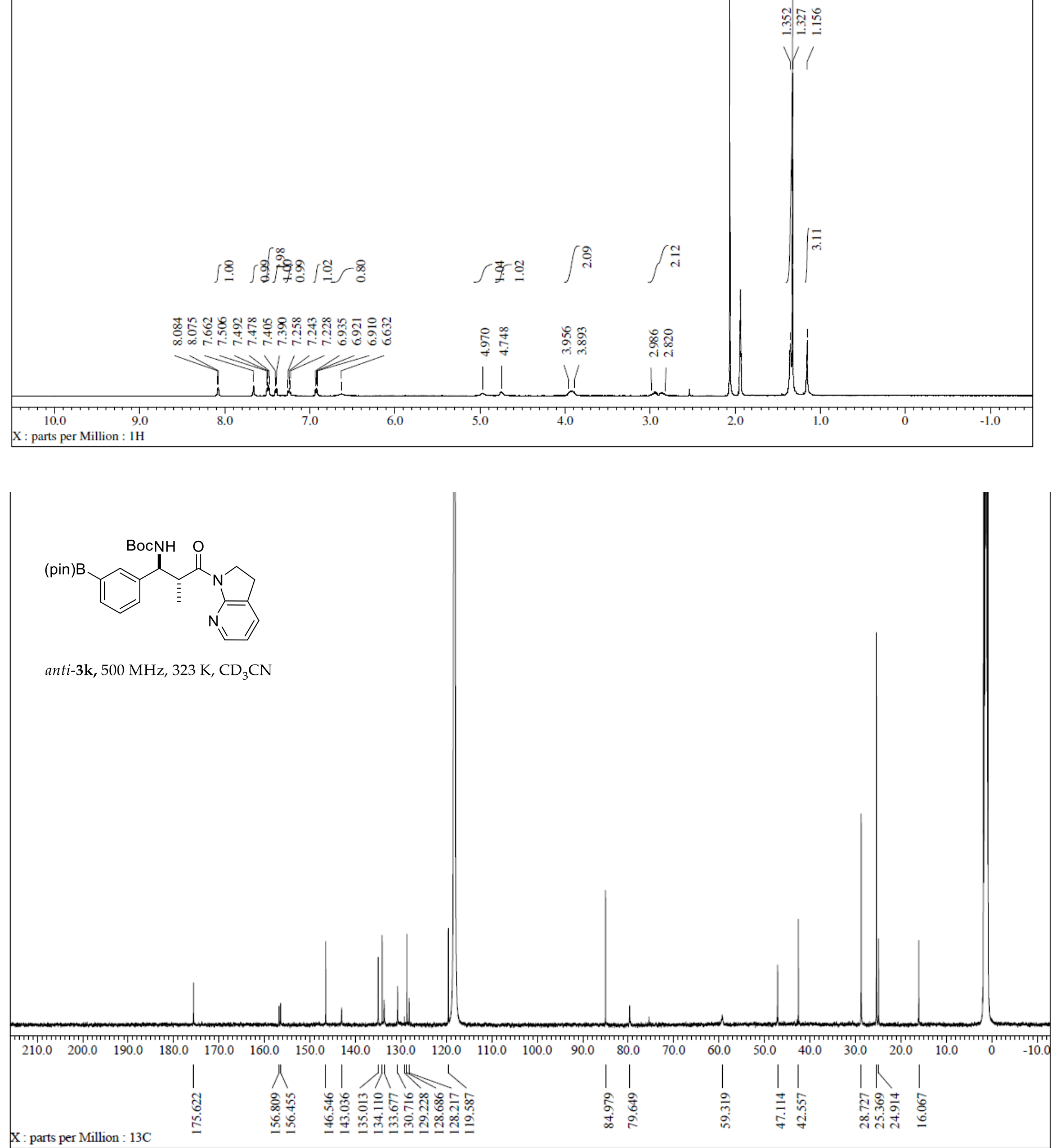
<smiles>CC(C(=O)N1CCc2cccnc21)C(NC(=O)c1ccccc1)c1ccoc1</smiles>

anti-31, $500 \mathrm{MHz}, 323 \mathrm{~K}, \mathrm{CD}_{3} \mathrm{CN}$
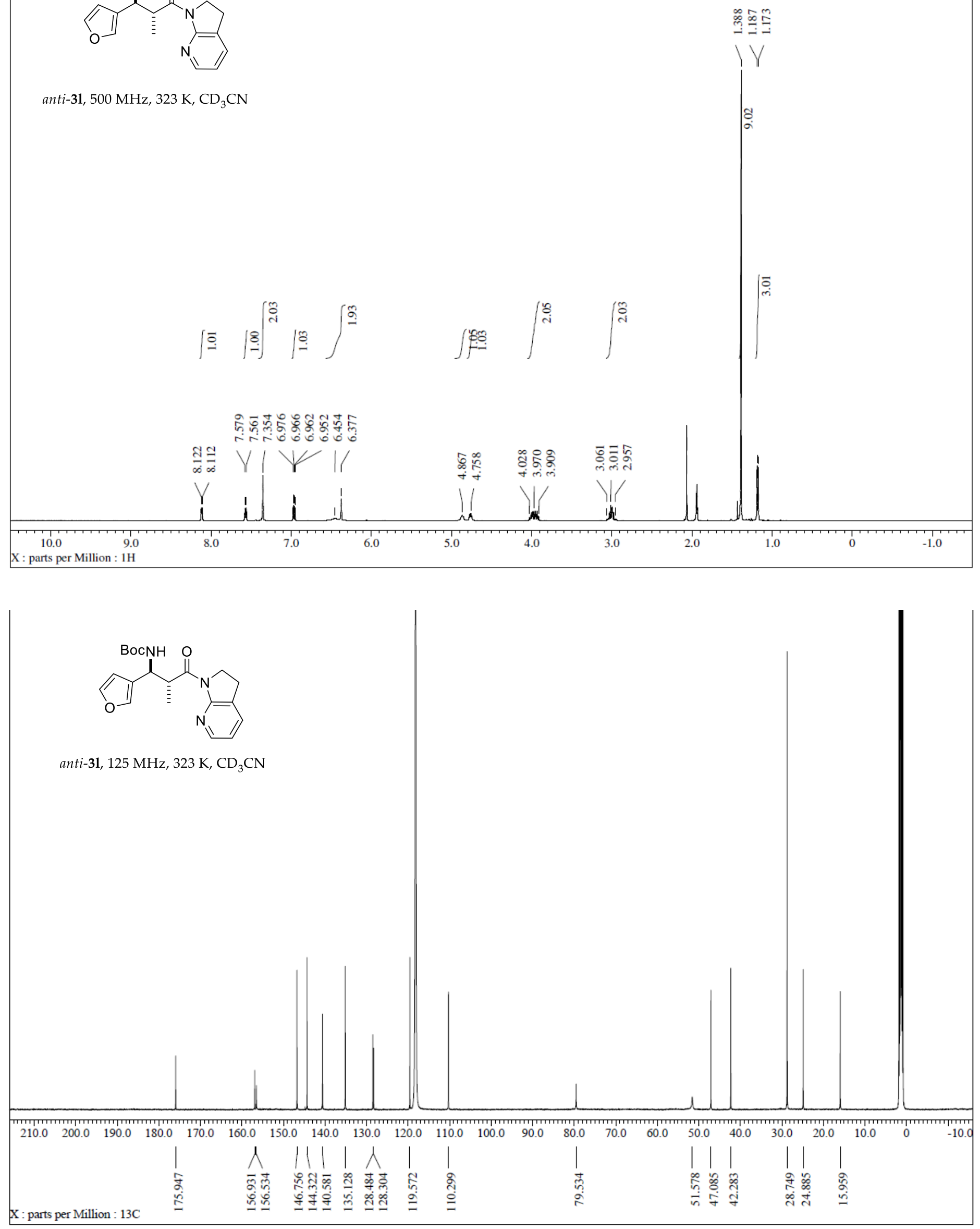
<smiles>C[C@H](C(=O)N1CCc2cccnc21)C(NC(=O)OC(C)(C)C)c1cccs1</smiles>

anti-3m, $400 \mathrm{MHz}, 323 \mathrm{~K}, \mathrm{CD}_{3} \mathrm{CN}$

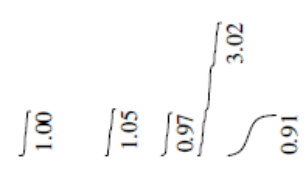

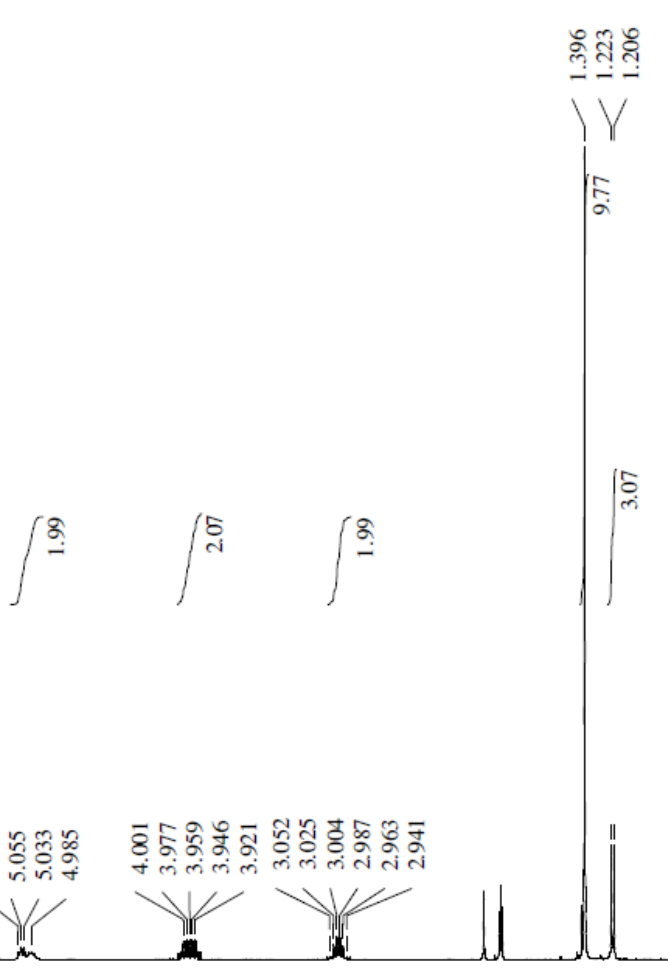

4.0

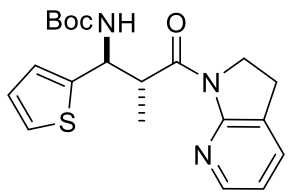

anti-3m, $100 \mathrm{MHz}, 323 \mathrm{~K}, \mathrm{CD}_{3} \mathrm{CN}$
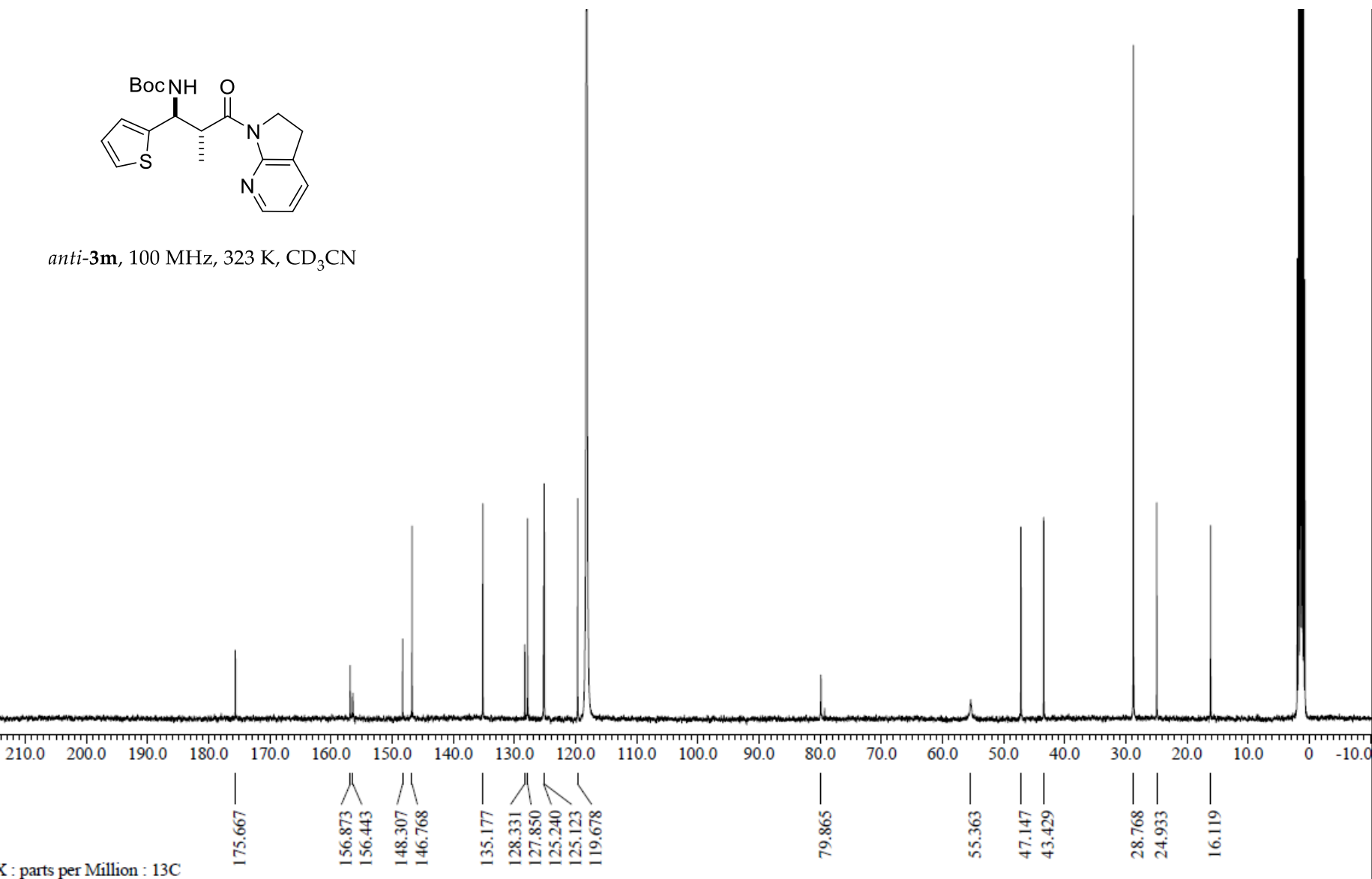


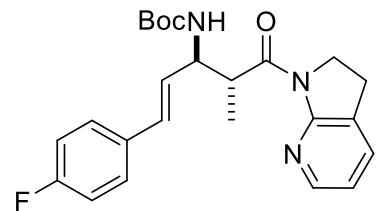

anti-3n, $500 \mathrm{MHz}, 323 \mathrm{~K}, \mathrm{CD}_{3} \mathrm{CN}$

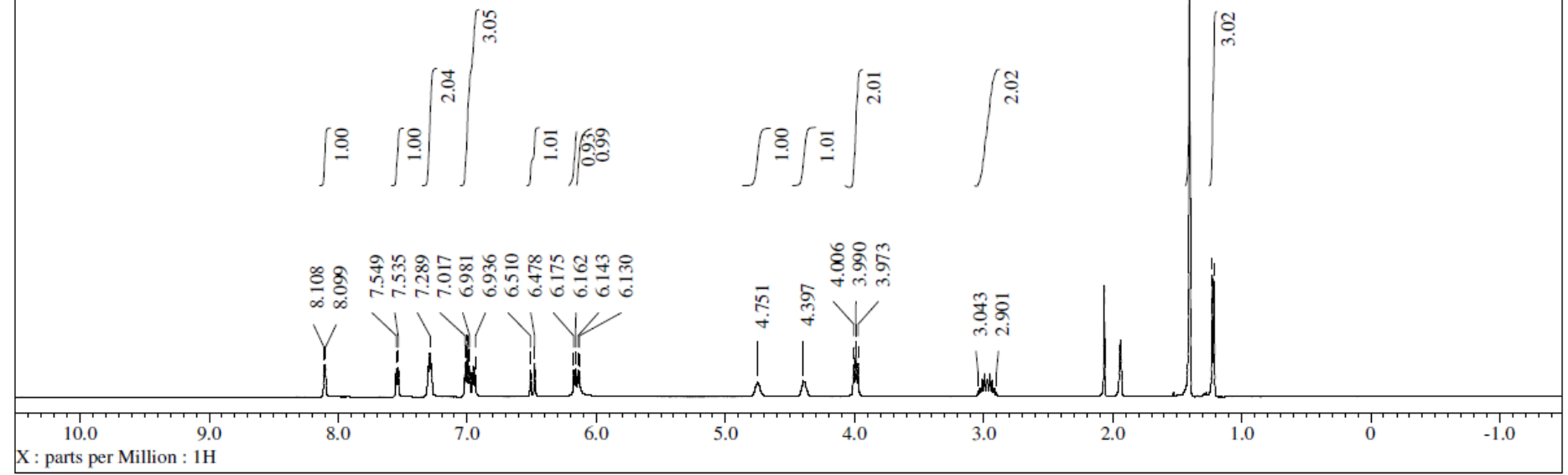

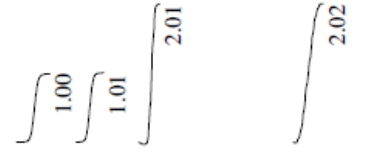

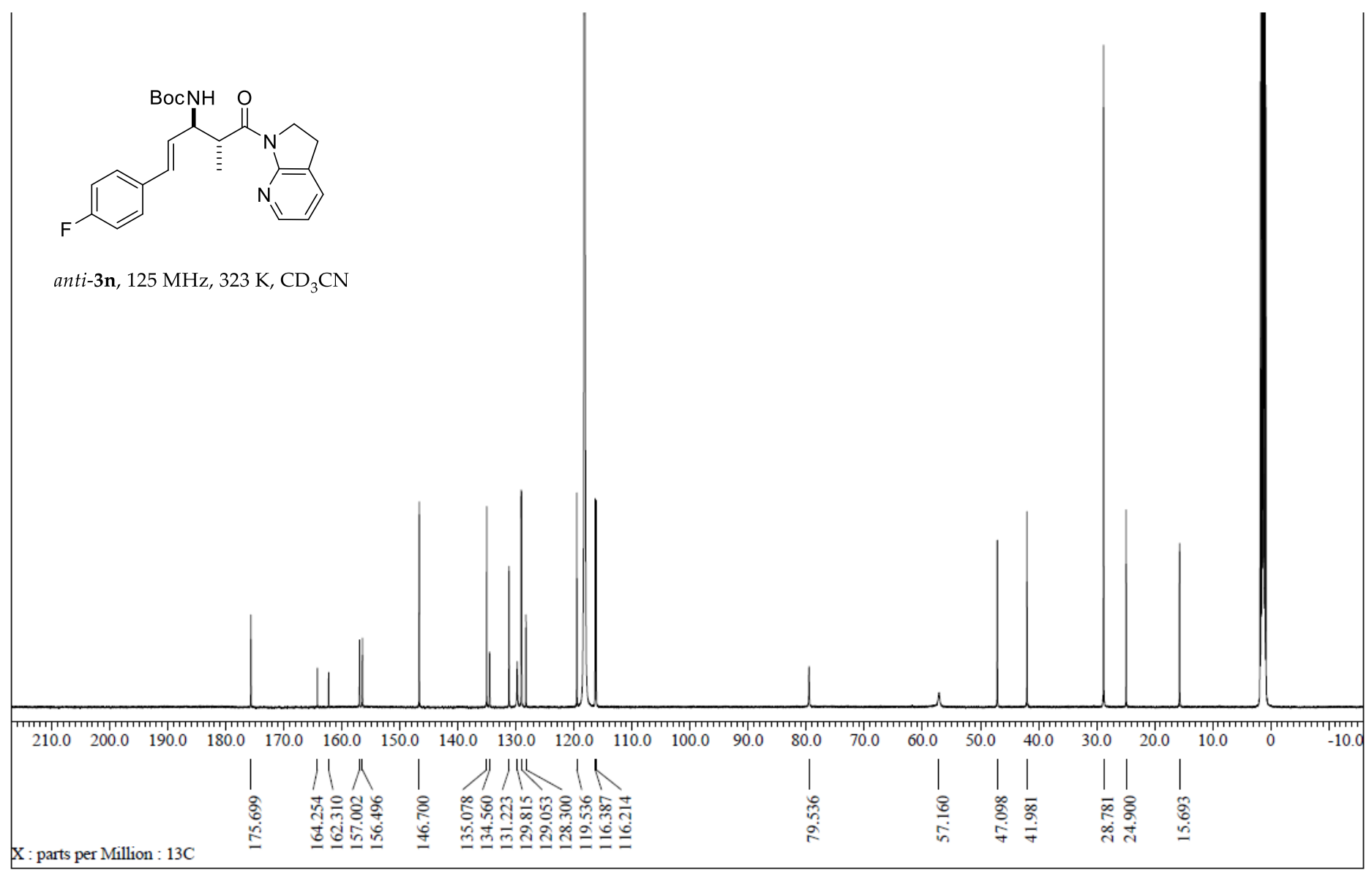



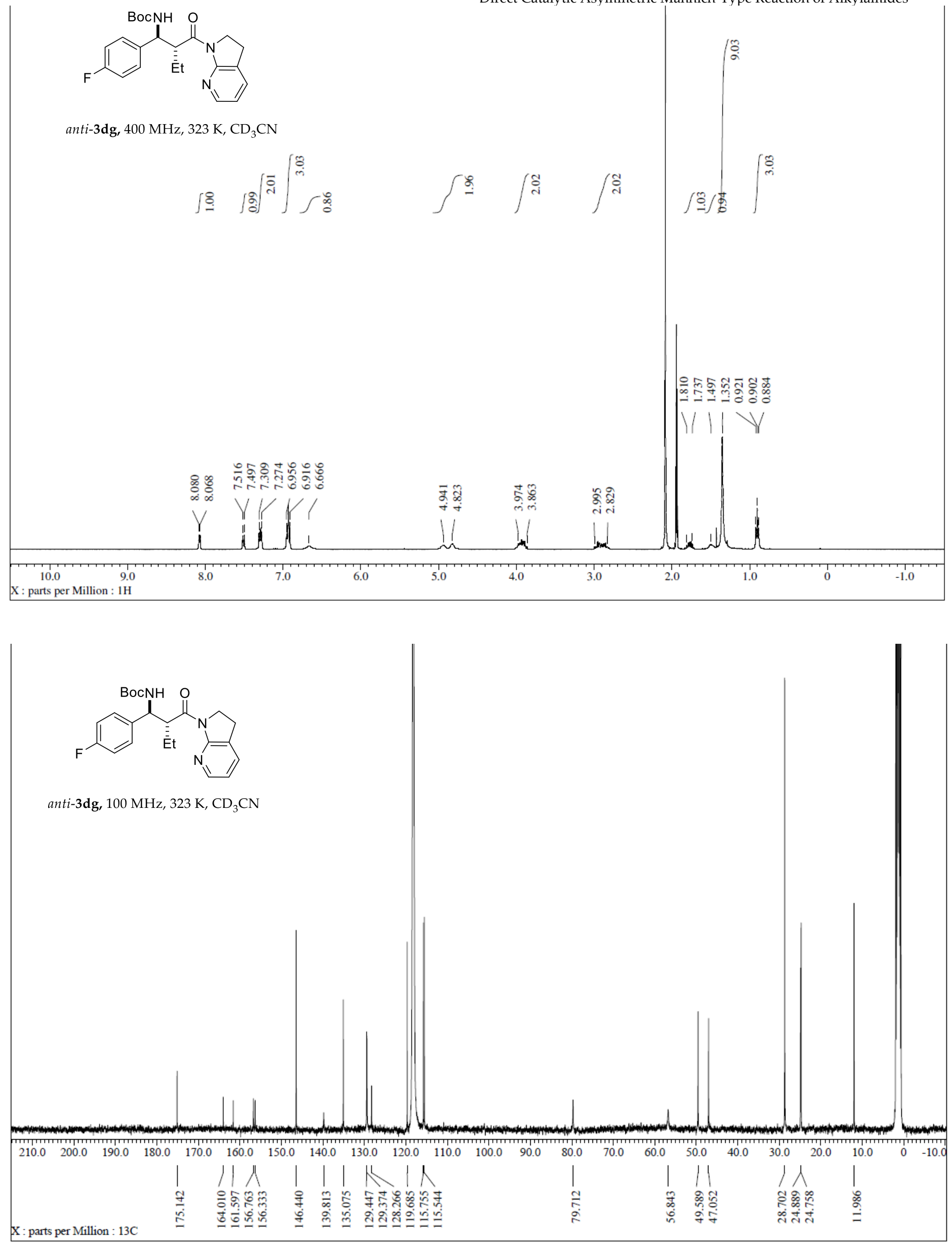


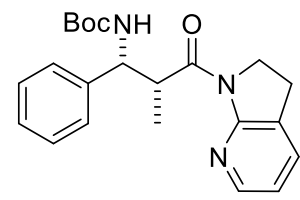

syn-3a, $400 \mathrm{MHz}, 323 \mathrm{~K}, \mathrm{CD}_{3} \mathrm{CN}$
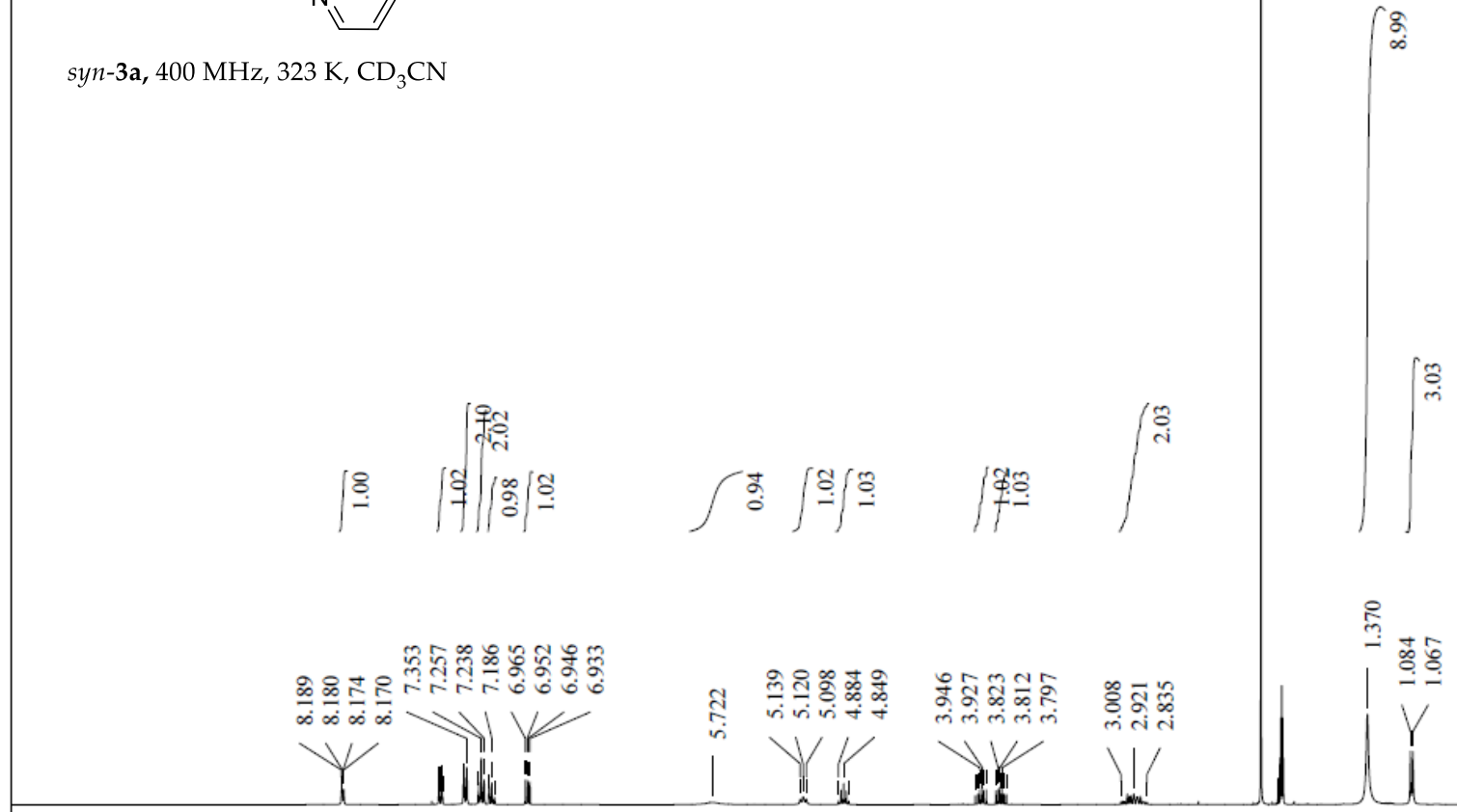

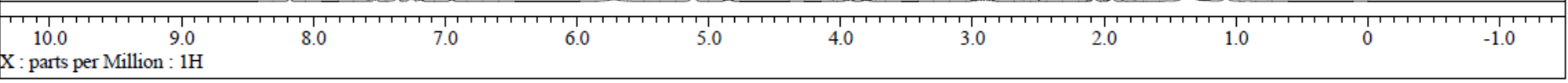

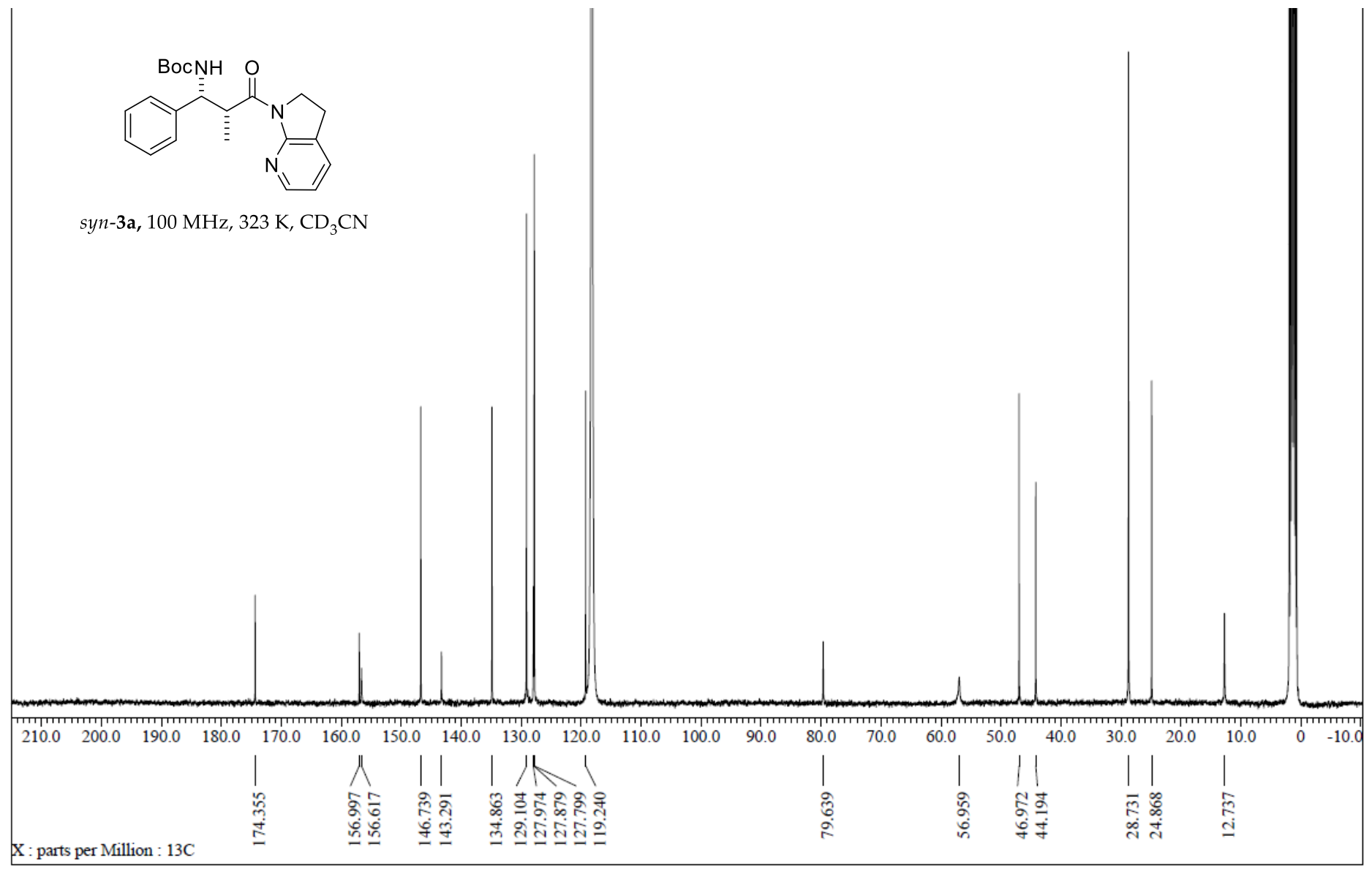


Supporting Information

Direct Catalytic Asymmetric Mannich-Type Reaction of Alkylamides
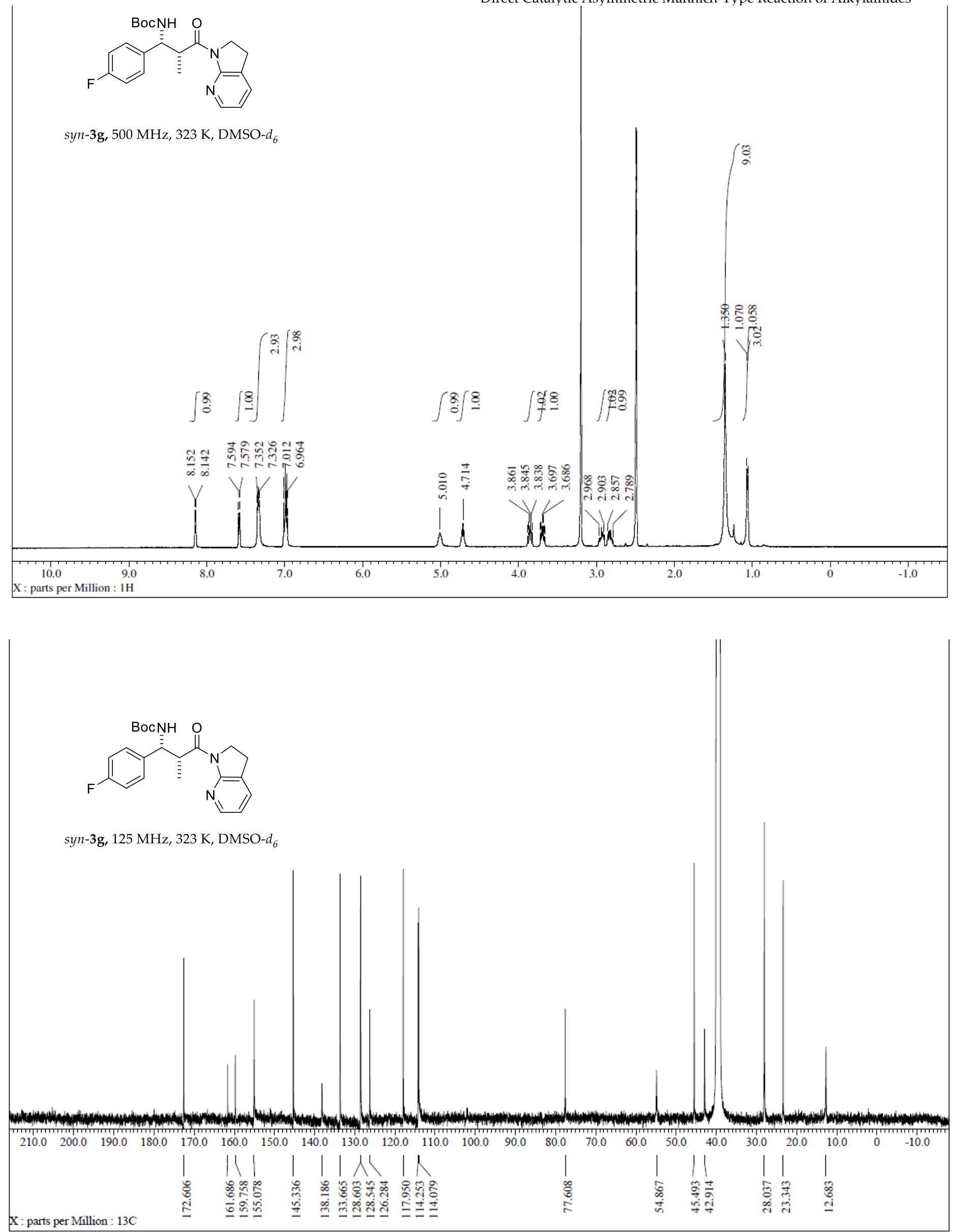


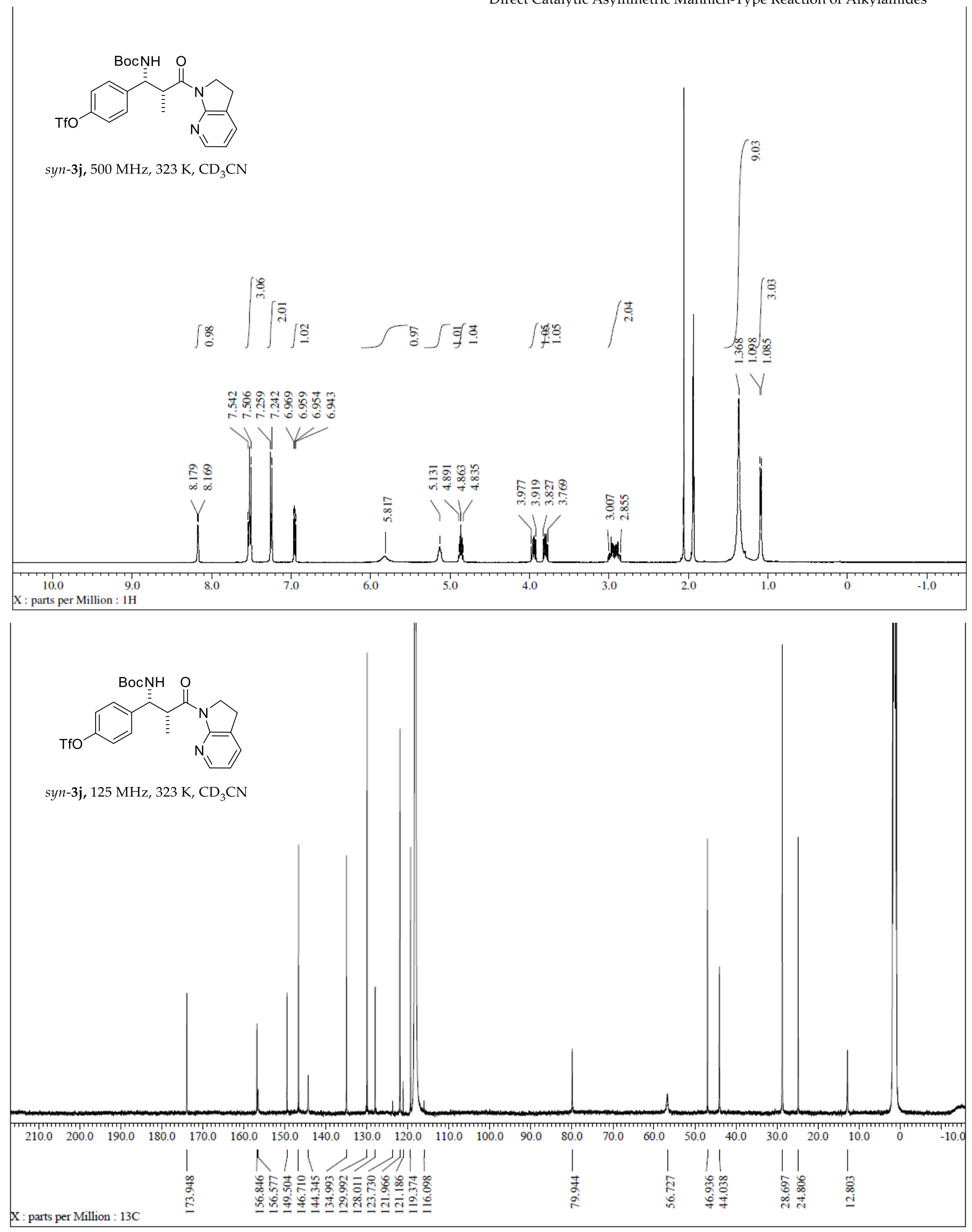



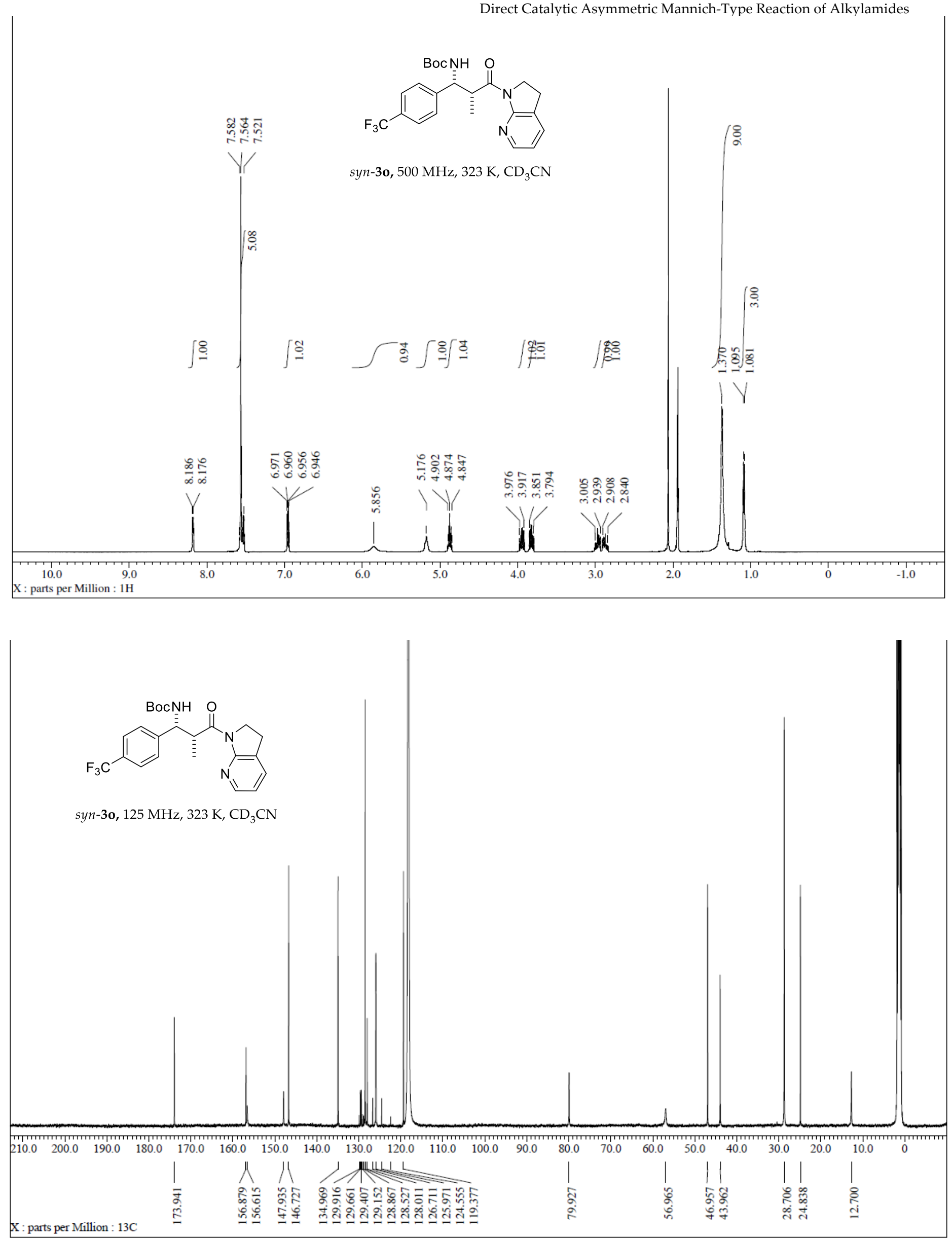


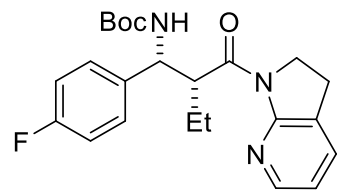

syn-3dg, $500 \mathrm{MHz}, 323 \mathrm{~K}, \mathrm{CD}_{3} \mathrm{CN}$
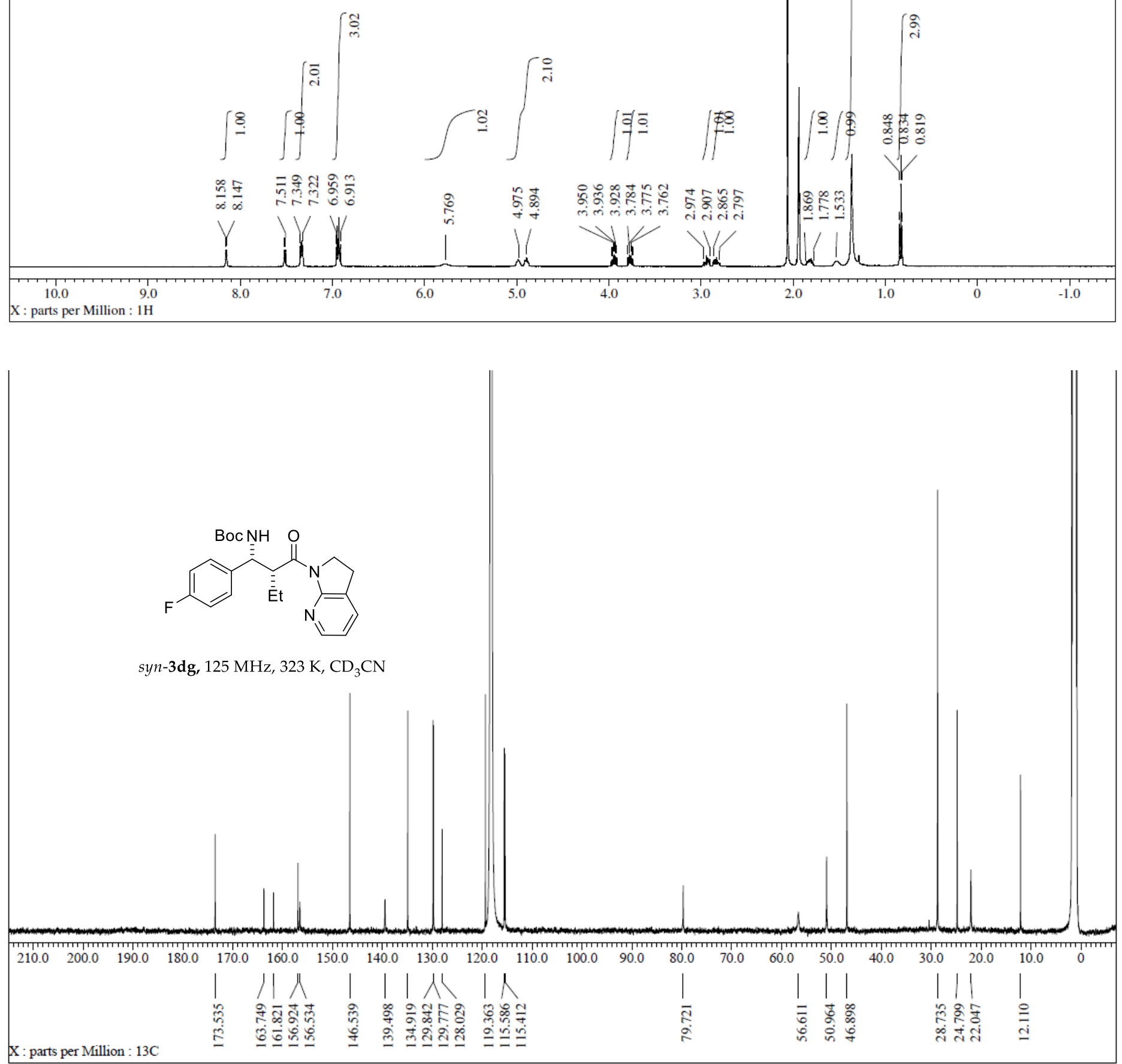


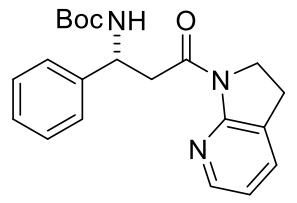

4a, $400 \mathrm{MHz}, 323 \mathrm{~K}, \mathrm{CD}_{3} \mathrm{CN}$
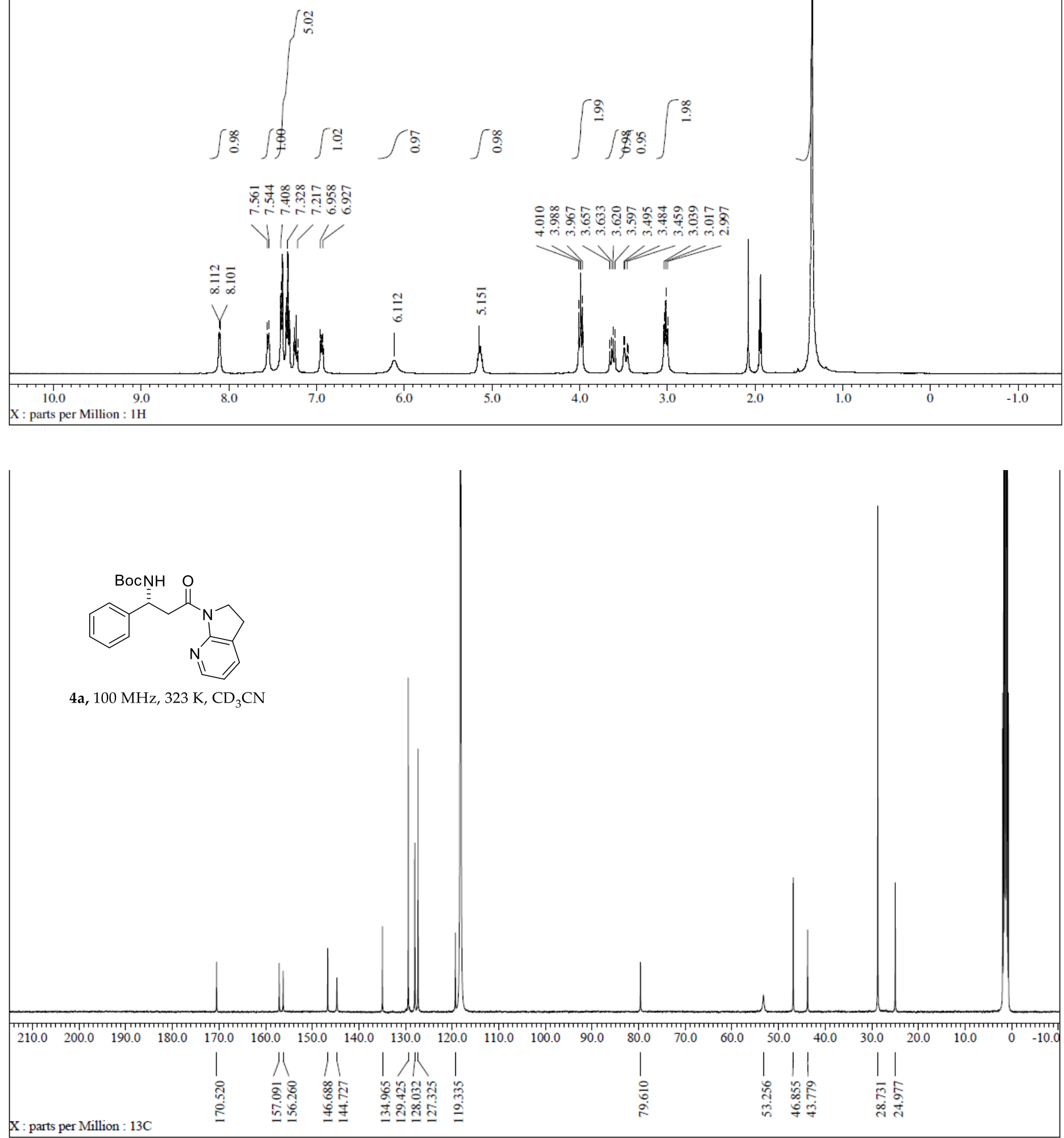
<smiles>Cc1ccc([C@H](CC(=O)N2CCc3cccnc32)NC(=O)OCc2ccccc2)cc1</smiles>

$4 \mathbf{b}, 400 \mathrm{MHz}, 323 \mathrm{~K}, \mathrm{CD}_{3} \mathrm{CN}$
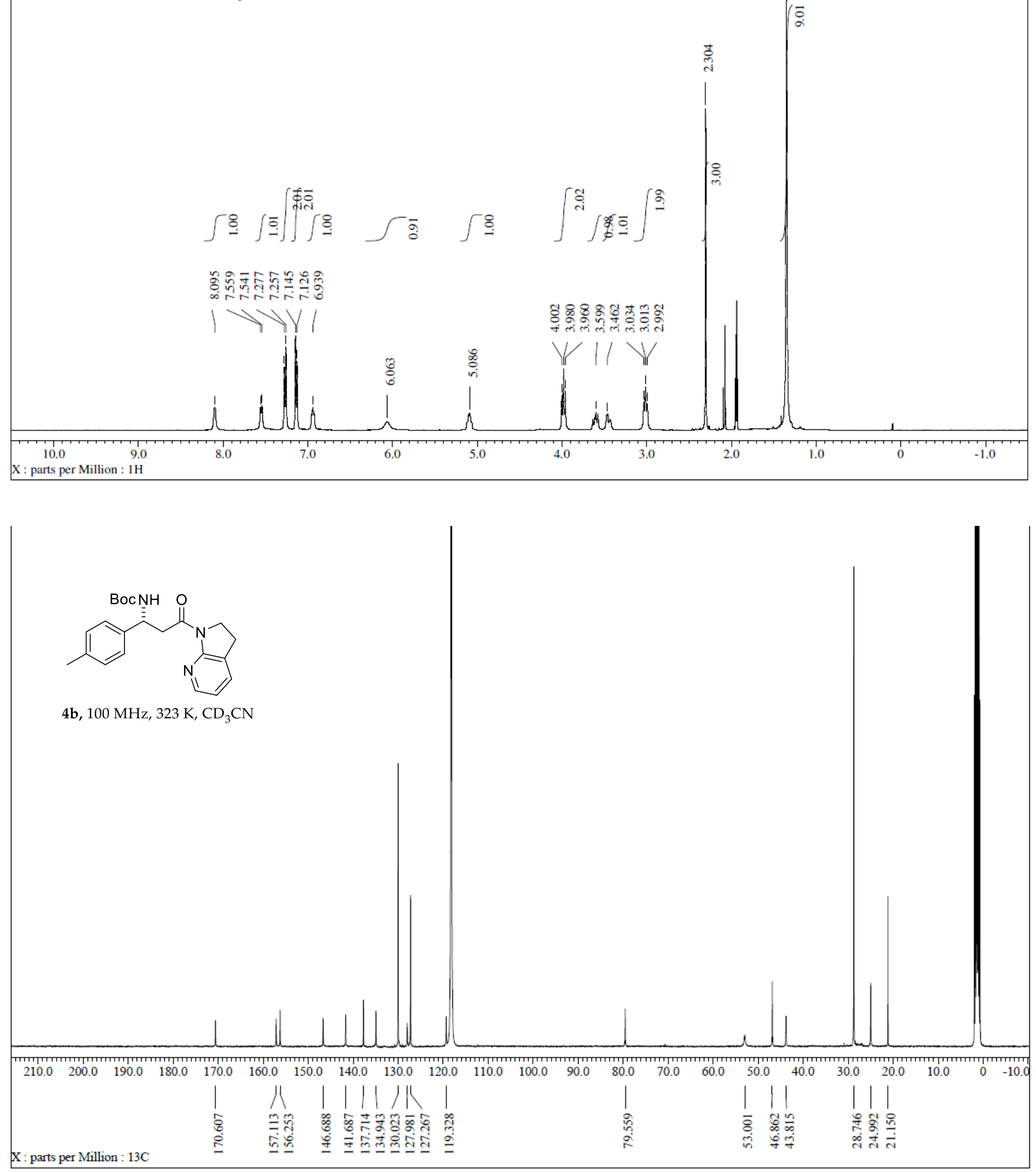


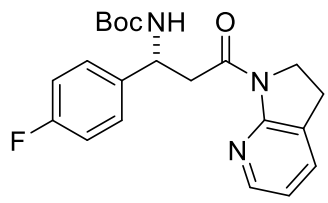

$4 \mathrm{~g}, 400 \mathrm{MHz}, 323 \mathrm{~K}, \mathrm{CD}_{3} \mathrm{CN}$

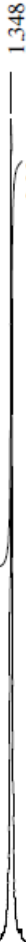

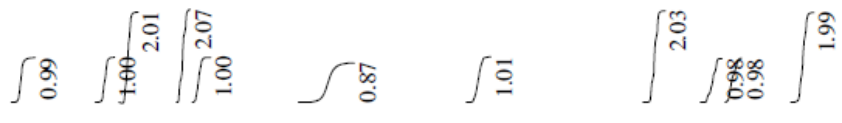
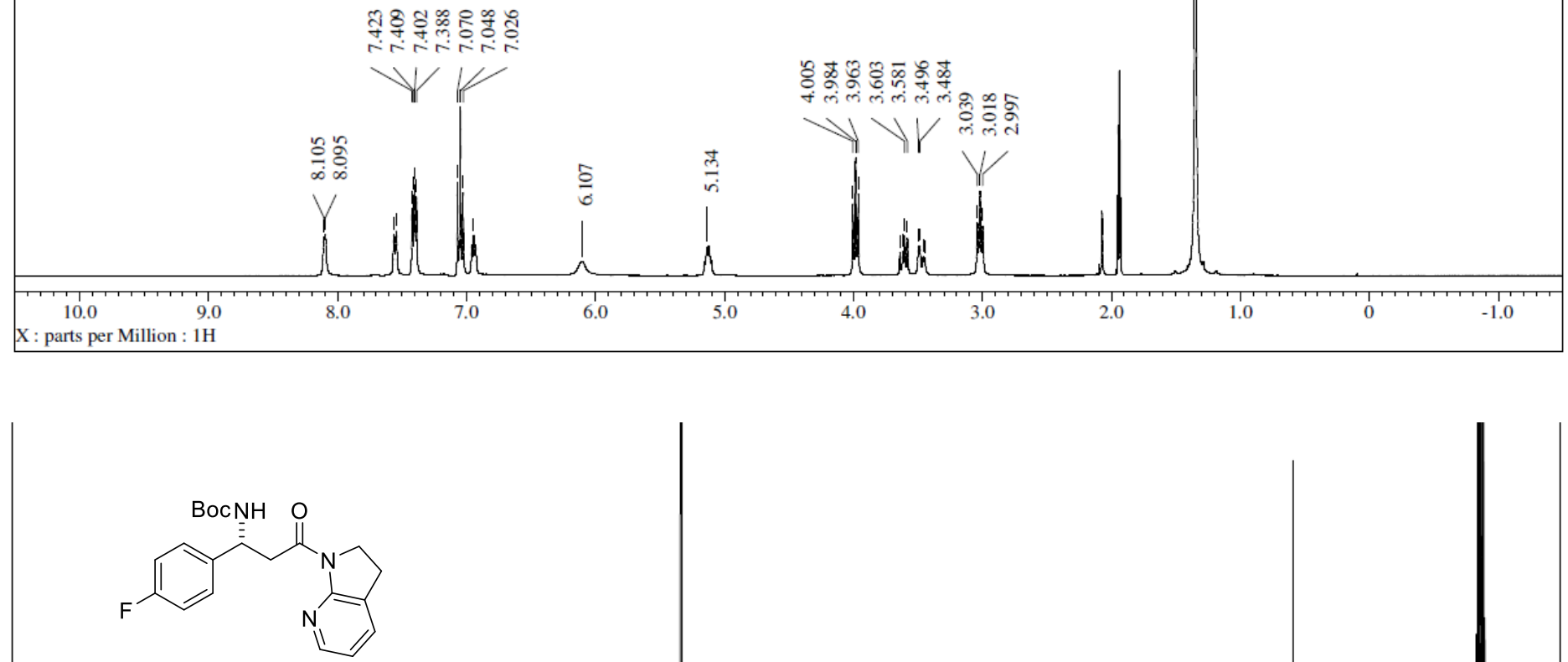

$4 \mathrm{~g}, 100 \mathrm{MHz}, 323 \mathrm{~K}, \mathrm{CD}_{3} \mathrm{CN}$

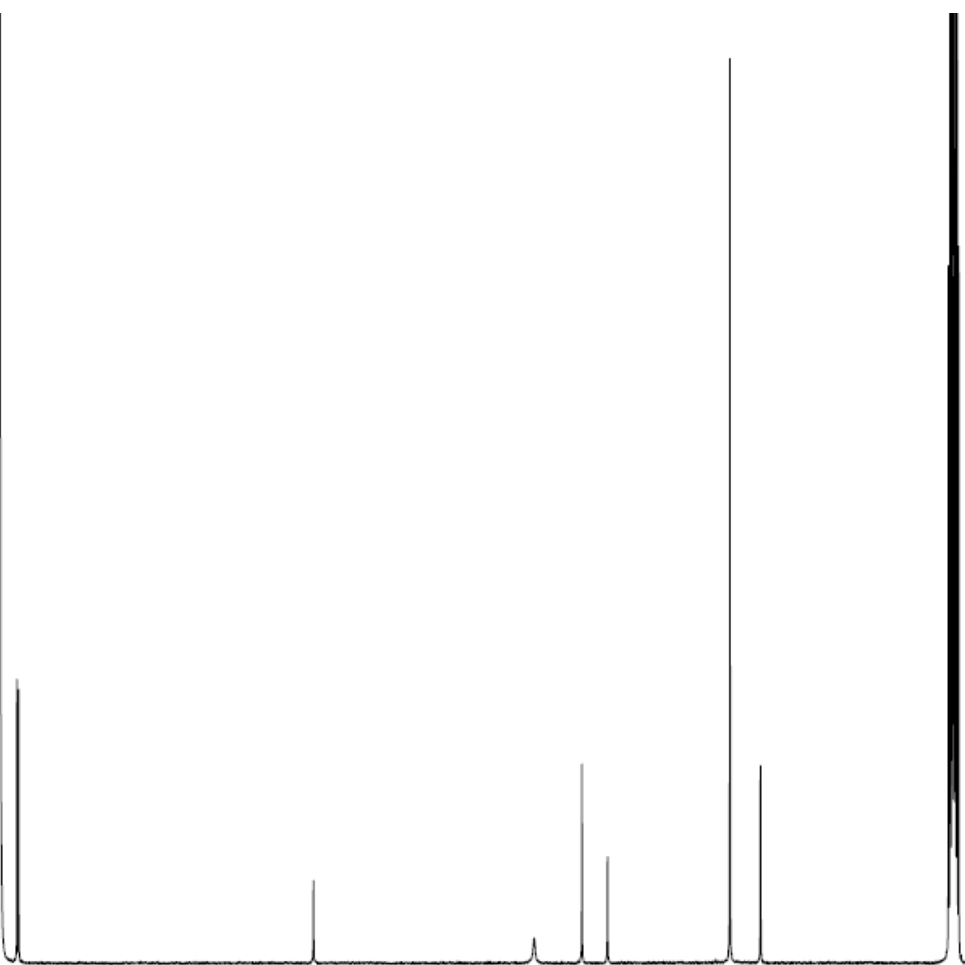

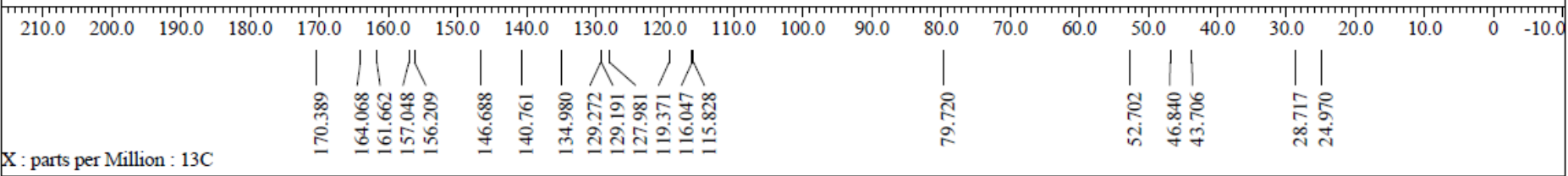




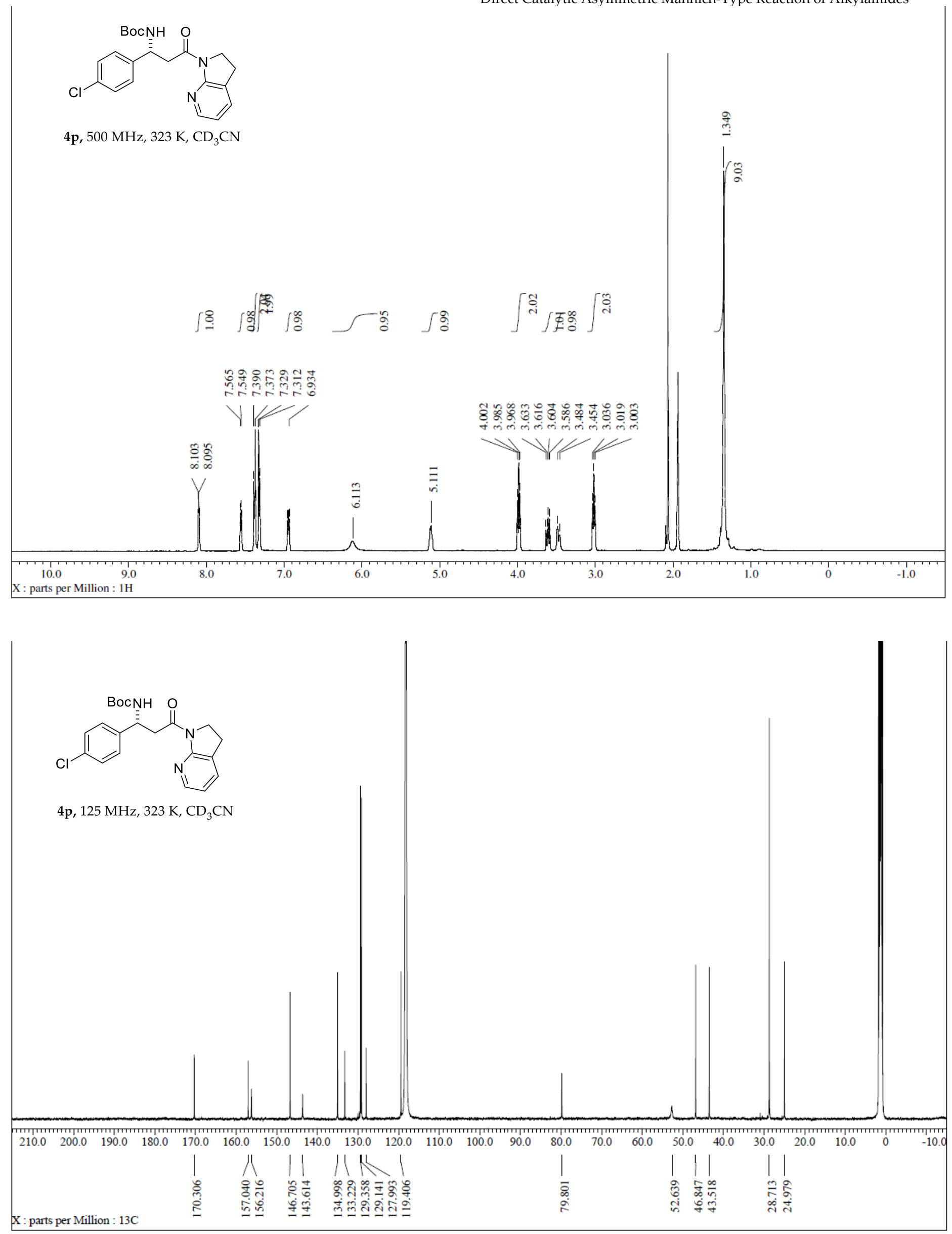


<smiles>C[C@H](C(=O)O)C(N)c1ccc(F)cc1</smiles>

5, $400 \mathrm{MHz}, 323 \mathrm{~K}, \mathrm{D}_{2} \mathrm{O}$

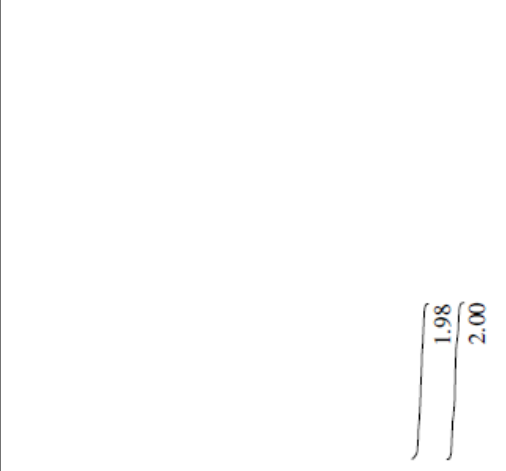<smiles>CC(C(=O)O)[C@H](N)c1ccc(F)cc1</smiles>

5, $100 \mathrm{MHz}, 323 \mathrm{~K}, \mathrm{D}_{2} \mathrm{O}$

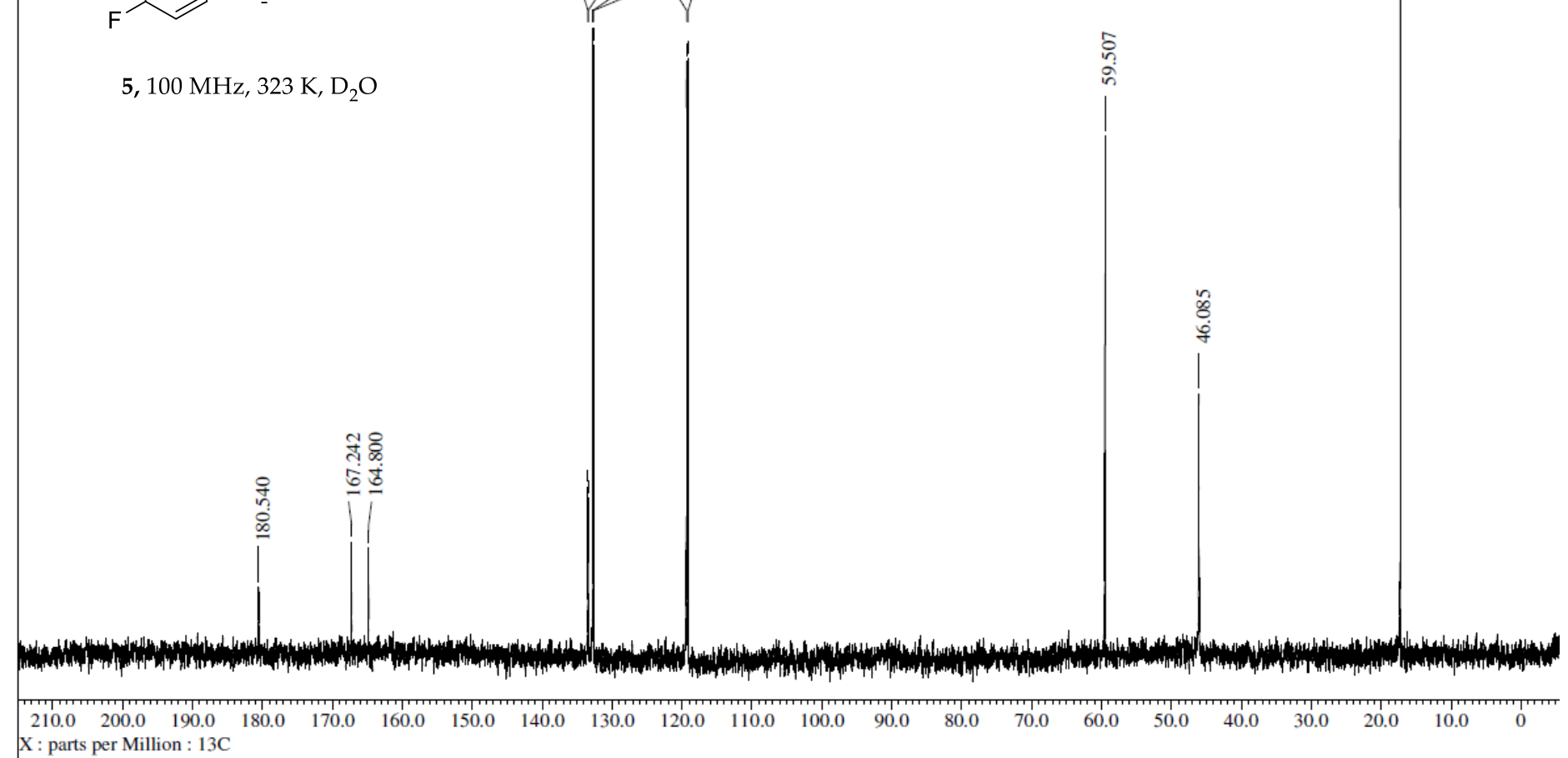


<smiles>C[C@H](C=O)C(NC(=O)OCc1ccccc1)c1ccc(F)cc1</smiles>

6, $500 \mathrm{MHz}, 323 \mathrm{~K}, \mathrm{CD}_{3} \mathrm{CN}$
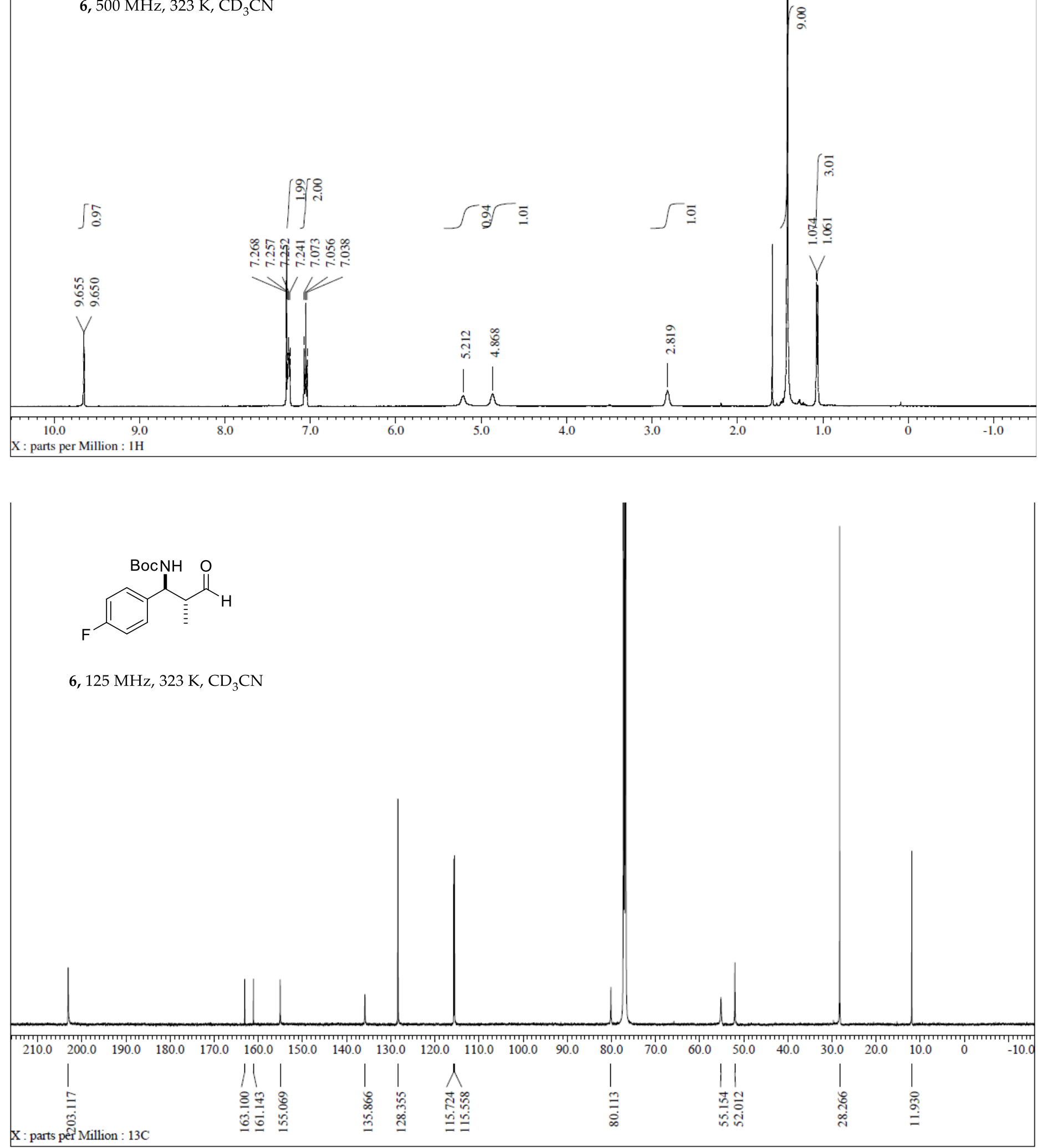
<smiles>C[C@H](C(=O)c1ccccc1)C(NC(=O)OCc1ccccc1)c1ccc(F)cc1</smiles>

7, $400 \mathrm{MHz}, 323 \mathrm{~K}, \mathrm{CDCl}_{3}$
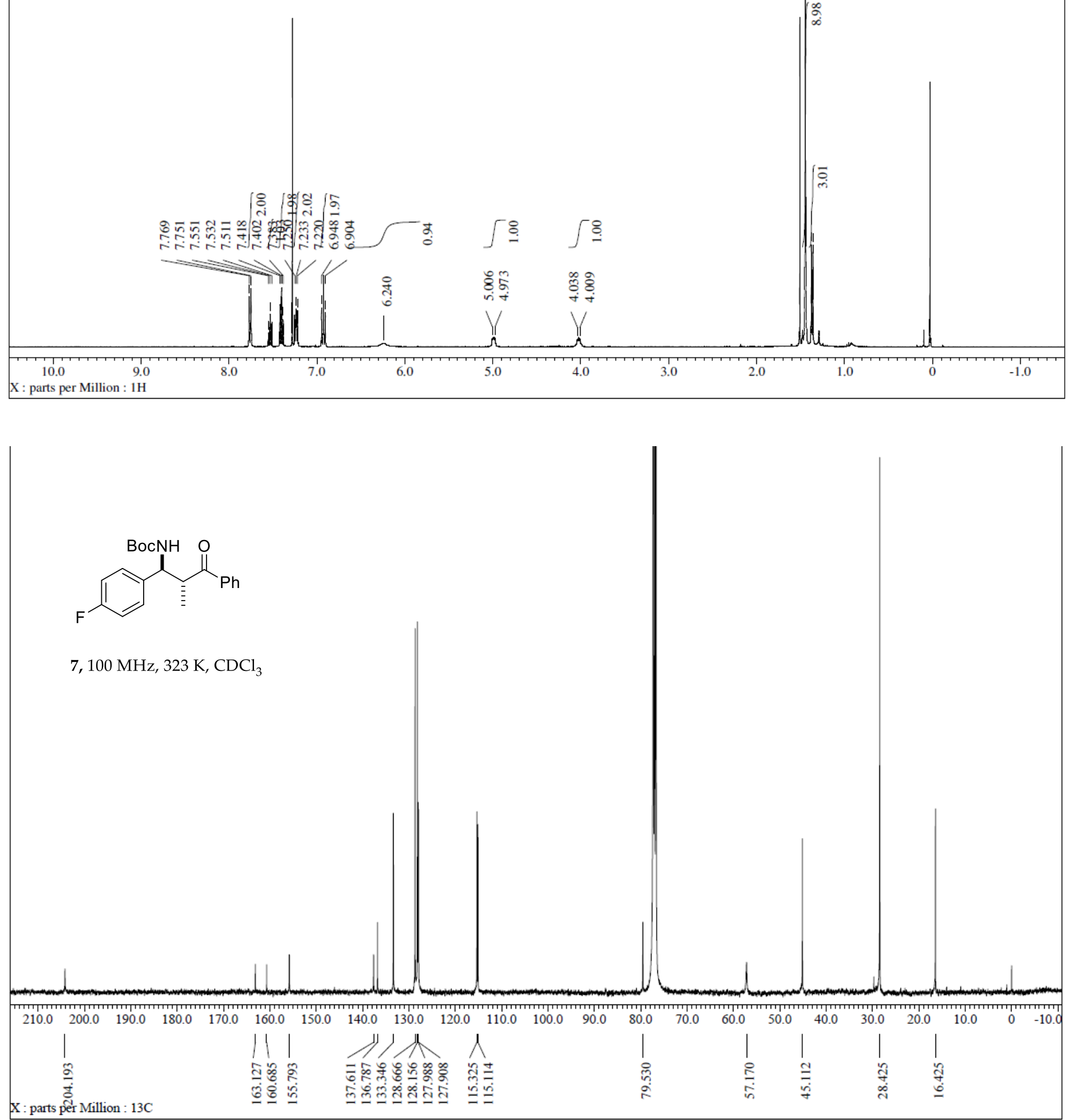
<smiles>C[C@H](CO)C(NC(=O)OCc1ccccc1)c1ccc(F)cc1</smiles>

8, $500 \mathrm{MHz}, 298 \mathrm{~K}, \mathrm{CD}_{3} \mathrm{CN}$
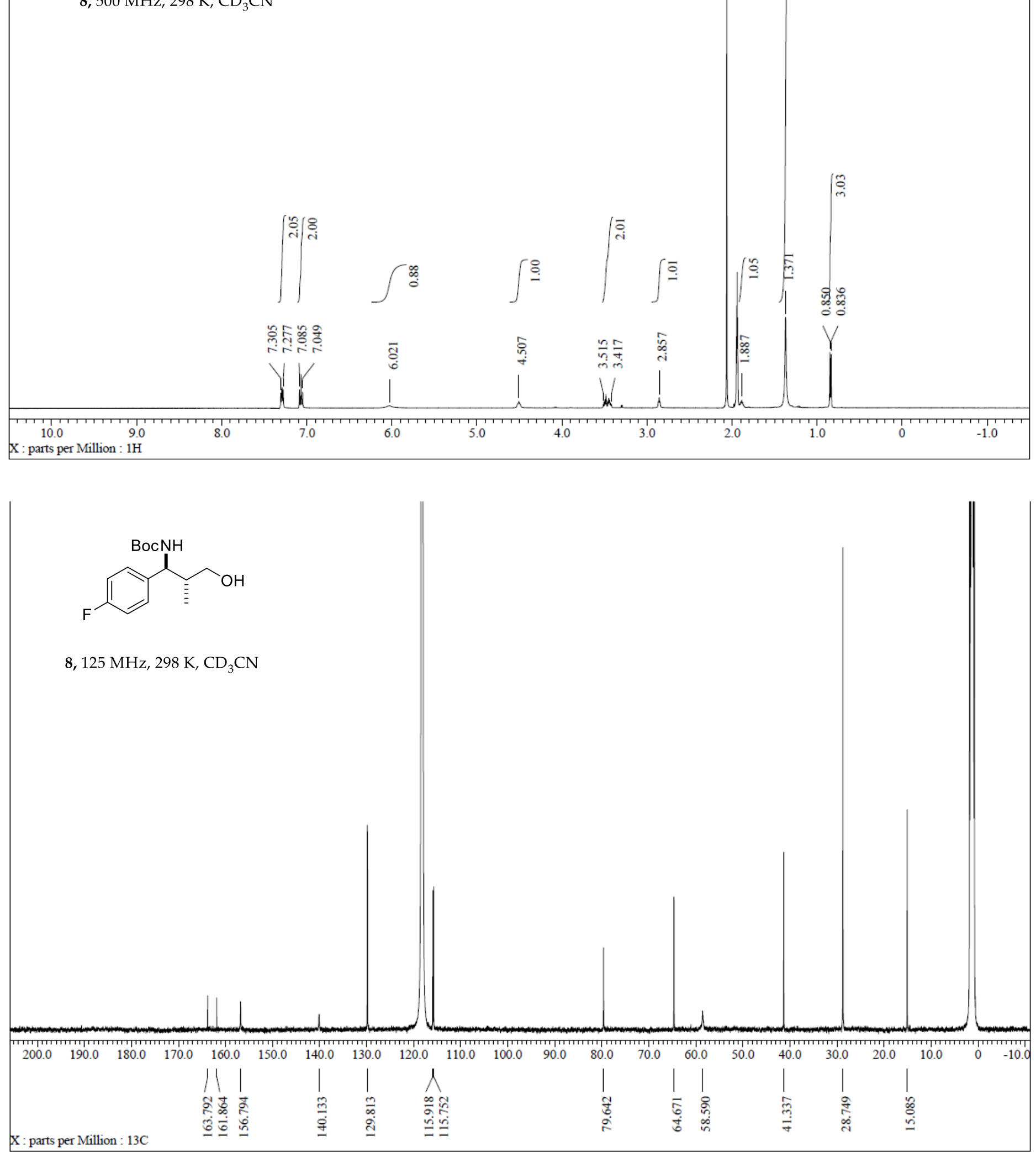